\title{
Two-dimensional transition metal dichalcogenides: Clusters, ribbons, sheets and more
}

Ruitao Lva ${ }^{\mathrm{a}}$, Humberto Terrones ${ }^{\mathrm{b}}$, Ana Laura Elías ${ }^{\mathrm{c}}$, Néstor Perea-López ${ }^{\mathrm{c}}$, Humberto R. Gutiérrez ${ }^{\mathrm{d}, 1}$, Eduardo Cruz Silva ${ }^{\mathrm{c}}$, Lakshmy Pulickal Rajukumar ${ }^{\mathrm{e}}$, Mildred S. Dresselhaus, ${ }^{\mathrm{f}, \mathrm{g}^{*}}$ and Mauricio Terrones ${ }^{\mathrm{c}, \mathrm{e}, \mathrm{h}^{* *}}$

${ }^{a}$ Key Laboratory of Advanced Materials (MOE), School of Materials Science and Engineering, Tsinghua University, Beijing, 100084, China

${ }^{\mathrm{b}}$ Department of Physics, Advanced Physics and Astronomy, Rensselaer Polytechnic Institute, 100 Eighth Street, Troy NY 12180, USA

${ }^{\mathrm{c} D e p a r t m e n t}$ of Physics and Center for 2-Dimensional and Layered Materials, The Pennsylvania State University, University Park, PA 16802, USA

${ }^{\mathrm{d}}$ Department of Physics \& Astronomy, University of Louisville, Louisville, KY 40292 USA

${ }^{\mathrm{e}}$ Department of Materials Science and Engineering, The Pennsylvania State University, University Park, PA 16802, USA, The Pennsylvania State University

${ }^{\mathrm{f}}$ Department of Electrical Engineering and Computer Science, Massachusetts Institute of Technology, Cambridge, MA 02139- 4307, USA

${ }^{\mathrm{g}}$ Department of Physics, Massachusetts Institute of Technology, Cambridge, MA 02139-4307, USA

${ }^{\mathrm{h}}$ Department of Chemistry and Materials Research Institute, The Pennsylvania State University, University Park, PA 16802, USA \& Carbon Institute of Science and Technology, Shinshu University, Wakasato 4-17-1, Nagano-city 380-8553, Japan

* Corresponding author. Tel.: +1-617-253-6864; fax: +1-617-253-6827.

${ }^{* *}$ Corresponding author. Tel.: +1-814-865-7533; fax: +1-814-865-3604.

E-mail address: millie@mgm.mit.edu (M. S. Dresselhaus), mut11@psu.edu (M. Terrones).

${ }^{1}$ Present address. Department of Physics, University of South Florida, Tampa, FL 33620, USA

\section{Summary:}

Monolayers of transition metal dichalcogenides (TMDs), such as $\mathrm{MoS}_{2}$ and $\mathrm{WS}_{2}$, have recently triggered worldwide research interest due to their remarkable optical and electronic properties. More fascinatingly is the fact that these monolayers could also adopt various morphologies with exposed edges that include triangular, hexagonal or star-shaped clusters, in addition to nanoribbons. Exciting progress has been recently achieved in the synthesis, characterization, device fabrication and functionalization of these monolayer and few-layer TMDs. This article firstly reviews the properties of bulk and monolayer/few-layer TMDs. The "top-down" and "bottom-up" synthesis routes for different TMDs are then summarized. Raman spectroscopy is now becoming a key tool 
used to characterize atomically thin TMDs, and this review will show the latest advances using this spectroscopic technique. Here we also summarize the most relevant characterization techniques, optical/electronic device fabrication, functionalization and defect engineering of TMDs. There are numerous opportunities for applications and multiple challenges to overcome, and this review will be instructive and useful to researchers working in the area of 2-Dimensional materials, as well as scientists and engineers interested in their applications in electronics, optics, catalysis, energy and many others.

KEYWORDS: Two-dimensional; Molybdenum disulfide; Tungsten disulfide; Monolayers; Few layers; Properties; Layered materials

\section{Introduction}

Layered materials consist of stacked sheets of atomically-thin systems, which are held together by van der Waals forces [1]. When these layers are exfoliated into monolayers or few-layer structures, new physical phenomena are found. For example graphene (i.e., monolayer graphite) has become the most popular 2-Dimensional (2D) material over the last decade, because of its unprecedented properties including room-temperature quantum Hall effect [2], very high room temperature carrier mobility (two orders of magnitude greater than that of silicon) [3], high room temperature thermal conductivity $(\sim 5000$ $\mathrm{W} /(\mathrm{m} \cdot \mathrm{K}))[4,5]$, and relatively high Young's modulus ( 1 TPa) [6]. Unfortunately, pristine graphene is also a zero band gap material, which limits its applications in some fields including semiconductor electronics. This drawback can be overcome by 
electrical/chemical doping $[7,8]$, or by searching for new alternative materials exhibiting an adequate electronic band gap and carrier mobility, and the feasibility of adequate on-off voltage for devices. In this context, and since other 2D inorganic materials could behave as insulators (e.g. $\mathrm{BN})$ or semiconductors $\left(\mathrm{ZnO}, \mathrm{MoS}_{2}, \mathrm{WS}_{2}, \mathrm{WSe}_{2}\right)$ and superconductors (e.g. $\mathrm{NbS}_{2}, \mathrm{NbSe}_{2}$ ), it is possible to achieve properties on demand, depending on the type of $2 \mathrm{D}$ materials; recent publications have summarized the rapid progress in the area of 2D materials [9-24].

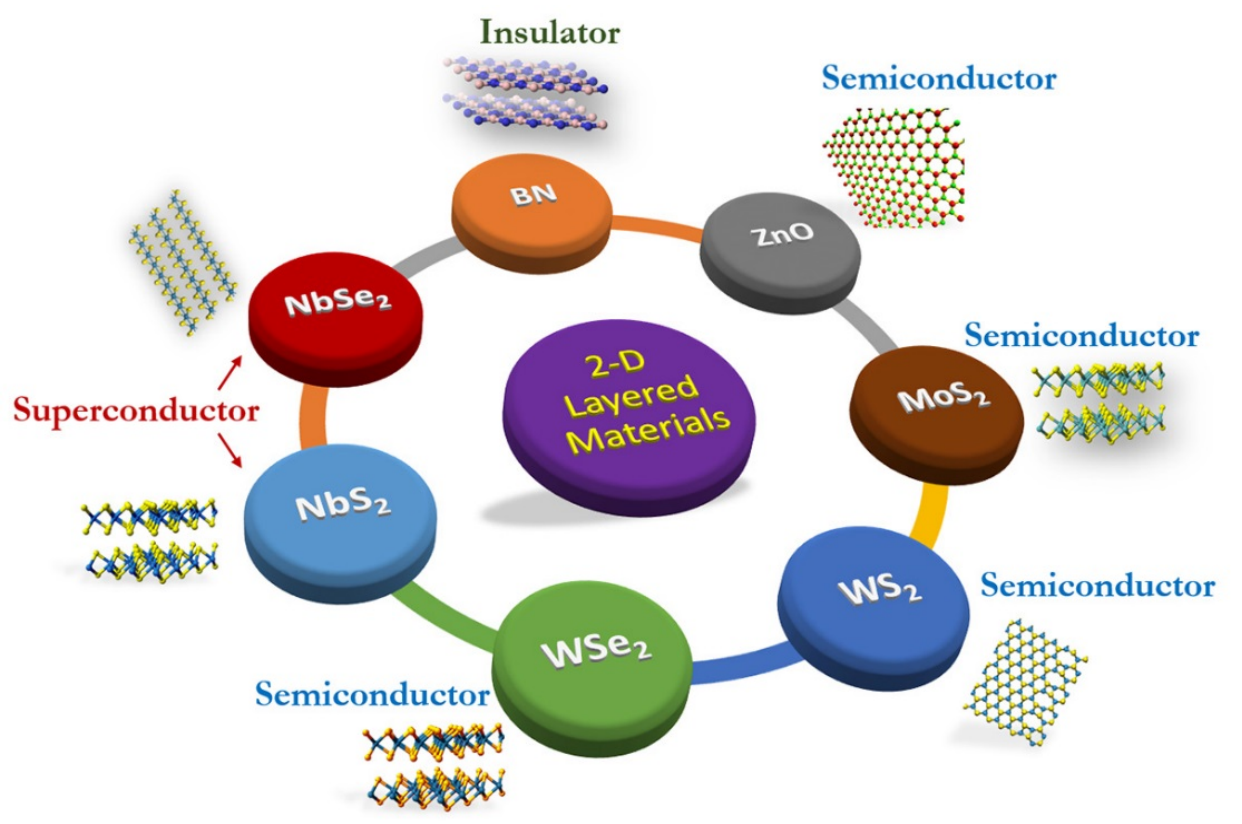

Figure 1 Diverse properties of different $2 \mathrm{D}$ materials. They could be insulators (e.g. $\mathrm{BN})$, semiconductors $\left(\mathrm{ZnO}, \mathrm{MoS}_{2}, \mathrm{WS}_{2}, \mathrm{WSe}_{2}\right)$ or superconductors (e.g. $\mathrm{NbS}_{2}, \mathrm{NbSe}_{2}$ ).

In this review, we focus on transition metal dichalcogenides (TMDs), which are compounds composed of a "sandwich" structure possessing a transition metal (e.g. Mo, W) layer in between two chalcogen layers (e.g. S, Se). In order to provide readers with a broad view of this fast growing field, we first introduce the bulk properties of TMDs, 
then show the fascinating structural, optical and electrical properties of monolayers and few-layer systems. A wide range of synthesis methods for producing TMD monolayers, clusters, sheets and nanoribbons will also be described. An emphasis on defects and their importance in controlling optical, catalytic and electronic properties will be then summarized. Finally, the challenges and future perspectives of this emerging field will be described.

\section{Bulk properties of transition metal dichalcogenides}

Since the 1960s, researchers tried to understand the properties of TMDs as a function of layers [25]. For example, $\mathrm{MoS}_{2}$ was exfoliated by mechanical cleavage (the so called "Scotch Tape Method"), and optical absorption studies were carried out at temperatures ranging from 120 to $175 \mathrm{~K}$ [25]. These early data indicated differences in the absorption spectra when varying the crystal thickness. In particular, a new peak, corresponding to the direct band gap of the material started to emerge at around $1.9 \mathrm{eV}$ when studying a crystal sample as small as $3 \mathrm{~nm}$. That peak was called "S", and its origin was unclear at that early time [25]. Although this review is mainly focused on few layered and monolayered TMDs, the importance of understanding bulk properties is fundamental for predicting the overall behavior of these materials. In section 2, we describe some of the bulk properties of TMD that have been studied for many decades and starting with section 3, we will focus on mono- and few-layered systems. 


\subsection{Photoelectrochemical properties}

As early as 1977 , the photoelectrochemical properties of TMDs started to be studied [26]. Semiconducting electrodes with photo-transitions in the visible spectral region appear to be suitable for electrochemical solar energy conversion applications. $\mathrm{MoS}_{2}$ (molybdenite) was the first material studied for this application, due to its natural availability when compared to other TMDs. Materials that can experience a phototransition without experiencing photodecomposition, such as $\mathrm{MoS}_{2}$ and $\mathrm{WS}_{2}$, represent an important advantage for solar energy conversion [26], and further studies using mono- and few-layered TMDs should follow. Soon after the pioneering report by Tributsch [26], the photoelectrochemical properties of other TMDs were also studied [27, 28]. Synthetic and bulk TMDs with transition metals that belong to groups IV, VI and VIII were studied and compared in an extensive report [28]. Materials such as $\mathrm{MoS}_{2}$, $\mathrm{MoSe}_{2}, \mathrm{MoTe}_{2}$ and $\mathrm{WSe}_{2}$ were soon found to be good candidates for solar cells [29, 30]. TMD compounds with $\mathrm{Zr}$ or Hf showed light-induced intercalation reactions suitable for their application in conversion and storage of solar energy. $\mathrm{PtS}_{2}$ was found to generate large photocurrents $\left(100 \mathrm{~mA} \mathrm{~cm}^{-1}\right)$ when compared to other TMDs from groups VI and VI, and $\mathrm{PtS}_{2}$ was proposed as a selective probe for electron exchanging substances.

A few years later, TMDs continued to attract the attention of numerous scientists due to their conversion efficiencies in photoelectrochemical devices, but, more importantly, due to their very high stabilities [31]. Interestingly, both $\mathrm{WSe}_{2}$ and $\mathrm{MoSe}_{2}$ showed higher efficiencies of solar to electrical energy conversion when compared to their sulfide counterparts [32]. An outstanding behavior was found particularly for $\mathrm{WSe}_{2}$, in which its stable output was found to be 400,000 coulombs $/ \mathrm{cm}^{2}$, without showing signs of 
photocorrosion [32]. Nowadays, the fabrication of van der Waals solids by stacking monolayers of different TMDs opens up new avenues for applications and the possibility to find layered systems with unprecedented photovoltaic properties. Moreover, by forming nanohybrids (e.g. CdS-WS ${ }_{2}$ ), monolayer TMDs can achieve high catalytic activity and enhanced stability in hydrogen evolution reactions (HER) [33].

\subsection{Thermoelectric properties of TMDs}

The constant search for new and efficient thermoelectric materials has motivated researchers to probe deeper into the thermoelectric properties of bulk transition metal oxides and TMDs [34]. Thermoelectric properties of intercalated and substitutionally doped layered compounds has been widely studied by numerous research groups [35, 36]. The earliest study on determining thermoelectrical properties of natural crystals of Molybdenite was reported by Mansfield and Salaam in 1953 [37]. These authors measured the electrical conductivity, Hall coefficient and thermoelectric power (or Seeback coefficient) of $\mathrm{MoS}_{2}$ over a wide temperature range $\left(-183{ }^{\circ} \mathrm{C}\right.$ to $\left.500{ }^{\circ} \mathrm{C}\right)$. From the Hall coefficient values obtained for different specimens, it was determined that the charge carriers were of $p$-type [37]. In 1954, Goldsmid et al. firstly demonstrated that $\mathrm{Bi}_{2} \mathrm{Te}_{3}$ can be used as a high-performance thermoelectric material which can achieve 0 ${ }^{\circ} \mathrm{C}$ cooling. They also utilized the thermoelectric quality factor to identify the high mobility, effective mass and the low thermal conductivity in semiconductors $[38,39]$.

A more extensive study in determining the Seebeck coefficient (S), electrical resistivity $(\rho)$, and thermal conductivity $(\kappa)$ for $\mathrm{AB}_{2}$ type Selenides and Tellurides of $\mathrm{Nb}$, Ta, Mo and W was carried out by Brixner in 1962 [34]. Using these values, the Figure of 
Merit (ZT) was computed in order to gauge their thermoelectric power conversion efficiencies. The highest ZT value was observed for $\mathrm{WTe}_{2}\left(\mathrm{ZT}: 7.08 \times 10^{-4}{ }^{\circ} \mathrm{C}^{-1}\right)$, closely followed by $\mathrm{WSe}_{2}\left(\mathrm{ZT}: 1.15 \times 10^{-4}{ }^{\circ} \mathrm{C}^{-1}\right)$. The ZT values calculated for $\mathrm{MoSe}_{2}, \mathrm{NbSe}_{2}$, and $\mathrm{TaSe}_{2}$ fell in the range between $1 \times 10^{-5}{ }^{\circ} \mathrm{C}^{-1}$ and $3 \times 10^{-5}{ }^{\circ} \mathrm{C}^{-1}$. The values of Seebeck coefficient (S) at room temperature, reported by Brixner corresponded to: $-780 \mu \mathrm{V}^{\circ} \mathrm{C}^{-1}$ for $\mathrm{MoTe}_{2},-60 \mu \mathrm{V}^{\circ} \mathrm{C}^{-1}$ for $\mathrm{WTe}_{2},-900 \mu \mathrm{V}^{\circ} \mathrm{C}^{-1}$ for $\mathrm{MoSe}_{2}$ and +700 to $+1000 \mu \mathrm{V}^{\circ} \mathrm{C}^{-1}$ for $\mathrm{WSe}_{2}[34,40]$. However, these results were not in agreement with the values later reported by Revolinsky and Beernsten in 1964 [41]. The major difference in both reports, apart from their values for $\mathrm{S}$, was that Brixner concluded $\mathrm{MoSe}_{2}$ and $\mathrm{MoTe}_{2}$ to be of $n$-type, whereas Revolinsky and Beernsten reported both these materials to be of $p$-type [41]. Therefore, there appears to have been a problem of unintentional doping and further studies on the sample preparation/source were needed to be carried out.

Brixner also investigated the dependence of the Seebeck coefficient (S) on temperature and this was found to vary for different materials and for different sources for the same material [34]. For example, the values of $\mathrm{S}$ increased with increasing temperature for $\mathrm{MoTe}_{2}$ and $\mathrm{WSe}_{2}$, whereas it decreased for $\mathrm{MoSe}_{2}$ and remained relatively constant for $\mathrm{NbSe}_{2}$ from 25 to $130{ }^{\circ} \mathrm{C}$. The value of $\mathrm{S}$ for $\mathrm{NbTe}_{2}$ was found to increase above $80^{\circ} \mathrm{C}$, following a change from $p$ to $n$ type. In an effort to study the effect of substitutional doping on the thermoelectric properties of Group VI TMDs, Hicks has obtained results that support Brixner's findings [42]. $\mathrm{MoSe}_{2}$ and $\mathrm{MoTe}_{2}$ were reported as being $n$-type materials at $100{ }^{\circ} \mathrm{C}$ but at higher temperatures $\left(600{ }^{\circ} \mathrm{C}\right) \mathrm{MoSe}_{2}$ converts from $n$-type to $p$-type. Hicks also observed that the thermal conductivity values for $\mathrm{MoSe}_{2}$ and $\mathrm{MoTe}_{2}$ 
are comparatively higher than that for $\mathrm{WSe}_{2}$, explaining why the latter shows an improved thermoelectric behavior [42].

It is interesting to note that different findings indicate an anisotropy in the thermoelectric properties of bulk TMDs [34, 43]. This stems from an anisotropy in the factors that contribute to thermoelectricity, such as thermal conductivity and electrical resistivity. Recent studies on the thermal properties of thin films of $\mathrm{WSe}_{2}(62 \mathrm{~nm}$ thick) reveal that their cross-plane thermal conductivity values are much smaller $(0.048 \mathrm{~W}$ $\mathrm{m}^{-1} \mathrm{~K}^{-1}$ at $300 \mathrm{~K}$ ) than the $c$-axis thermal conductivity, and also a factor of 6 times smaller than the smallest value predicted for this material [44]. This is believed to arise from a random stacking of different crystallites of $\mathrm{WSe}_{2}$ which localizes lattice vibrations in these materials. It has also been mentioned in the same report, that the electrical conductivity in the cross-plane direction of as-synthesized $\mathrm{WSe}_{2}$ films is still poor. If their electrical conductivity can be improved, it will be ideal for obtaining a material with good thermoelectric energy conversion efficiencies. The disorder impedes localization of vibrational energy, thus promoting transfer of this energy into the material [44].

\subsection{Tribological properties of TMDs}

The crystal structure of layered materials, in which covalently-bonded atomic planes are stacked and held together by weak van der Waals forces, results in low shear resistance that confers them excellent lubricating properties to certain layered materials in their solid state. Among these materials, graphite has been known to be an excellent solid lubricant. However, graphite's electric conductivity has an adverse effect on applications involving metals, since graphite promotes galvanic corrosion in them [45]. It has also 
been found that the friction coefficient and the wear on graphite are dramatically increased under vacuum conditions, due to the lack of adsorbed condensates, which are needed to decrease the interaction between adjacent graphite's layers [46, 47].

TMDs exhibit a crystal structure similar to that of graphite, and each TMD layer consists of a plane of transition metal atoms sandwiched between two layers of chalcogen atoms (see above). The interlayer van der Waals interaction drives the good stacking of adjacent layers, thus resulting in good lubrication properties for TMDs [48]. Unlike graphite, TMDs can be semiconductors and hence do not promote galvanic corrosion, and their thermal stability make them excellent candidates for high temperature applications [49]. TMDs could be disadvantageous when lubrication is required in an oxidative or humid environment, because these contaminants have been shown to promote the conversion of sulfides into oxides, thus promoting a higher friction coefficient and consequently an increased wear rate of the lubricant surfaces [50]. By studying the friction coefficient of compressed pellets of $\mathrm{MoS}_{2}$ and $\mathrm{WS}_{2}$ powders under vacuum, it was early realized that $\mathrm{MoS}_{2}$ and $\mathrm{WS}_{2}$ preserved their lubricant properties under vacuum due to a self-lubricating process in which amorphous sulfur builds up on the crystal surface as a result of the initial friction, resulting in a fast decay of friction into a low steady-state at room temperature [51,52]. Similar properties were found for titanium and zirconium sulfides, selenides, and telurides [53]. Besides the formation of the amorphous sulfur condensates [52], high-resolution transmission electron microscopy (HRTEM) studies of $\mathrm{MoS}_{2}$ films during friction measurements under ultrahigh vacuum, revealed Moire patterns consistent with a rotational misfit of the $\mathrm{MoS}_{2}$ layers in the [0001] direction; responsible of creating a superlubric state caused by the incommensurability of 
the lattice when subjected to rotational disorder [54].

While a low friction coefficient of TMD coatings is needed for solid lubricant applications, it is as important for these materials to be wear resistant in order to increase their expected lifetime. Therefore, several efforts have been made in order to improve the hardness of TMDs while at the same time provide protection against environmental pollutants, such as oxygen or water vapor. In particular, multi-layered $\mathrm{MoS}_{2} / \mathrm{WS}_{2}$ films with a total film thickness of $500 \mathrm{~nm}$ and a hybrid layer period of $10 \mathrm{~nm}$ (thickness of each layer is $5 \mathrm{~nm}$ ), deposited by radio frequency sputtering, display a reduction of $c a$. $60 \%$ of both the wear rate and the indentation depth when compared to either $\mathrm{MoS}_{2}$ or $\mathrm{WS}_{2}$ thin films of the same total thickness [55]. Other approaches for achieving good lubrication surfaces include multi-layer structures with metallic layers, such as $\mathrm{MoS}_{2}$ in combination with $\mathrm{Cr}$ [56], $\mathrm{Ti}[56,57], \mathrm{Ni}$, and $\mathrm{Au}-\mathrm{Pd}$ [58], among others.

\section{Monolayer and few-layer TMD properties}

In all crystalline materials, surface effects exhibit particular properties that are different from bulk phases, and Van der Waals solids are not the exception. In fact, due to the weak interaction between layers, the exfoliation in these materials is possible in such a way that electronic and optical properties can be monitored as the layer number increases from monolayers to the bulk. As explained in the previous sections, bulk semiconducting trigonal prismatic (2H phases) TMDs possess an indirect band gap behavior which changes with deceasing number of layers, and when reaching a monolayer the system exhibits direct electronic and optical band gaps [59-61]. Moreover, TMDs could also exhibit a metallic behavior in the bulk and in monolayers, thus resulting 
in alternative applications of these fascinating 2-D materials which can also exhibit magnetic [62-64] and superconducting [65-67] behavior. In this section the main experimental properties and theoretical results of few-layer TMDs are summarized.

\subsection{Experimental evidence and properties of single and few layer semiconducting TMDs}

As in graphene and hexagonal boron nitride (h-BN), the idea of making atomically thin TMD is not new, and the seminal work of Frindt et al., using adhesive tape exfoliation $[25,68]$ and chemical exfoliation by lithium intercalation [69], have become the first efforts to reach monolayered TMDs. Specifically, in 1965 and in 1966 Frindt used the adhesive tape (or "Scotch tape") method to produce thin $\mathrm{MoS}_{2}$ crystals with different thicknesses varying from $500 \AA$ to $15 \AA$. In his work of 1965 , the optical absorption of relatively thin $\mathrm{MoS}_{2}$ layers up to $25 \AA$ thick (four molecular layers thick) was reported [25]. The authors analyzed the exciton behavior by varying the crystal thickness at different temperatures and found that the B exciton occurring $\sim 2.09 \mathrm{eV}(592.5 \mathrm{~nm})$ changes little in intensity with thickness, while excitons A (around $1.89 \mathrm{eV}$ or $654.5 \mathrm{~nm}$ ), and $\mathrm{A} 1, \mathrm{~A} 2$ and $\mathrm{A} 3$ (which appear for thick samples), get weaker when the samples get thinner [25]. Interestingly. exciton shifts to higher energies have also been found as the crystal thickness decreases in chemical vapor transport crystals of tape exfoliated $\mathrm{WSe}_{2}$, up to thicknesses of $40 \AA$ at $77 \mathrm{~K}$ [70]. In fact, this behavior is also observed in the other semiconducting TMDs and was recently confirmed by first principles calculations [71]. Therefore, as the crystal decreases in thickness the direct optical gap becomes dominant.

In 1987, Coehoorn, Hass and Groot, using angle resolved ultraviolet photo-electron 
spectroscopy (ARUPS), confirmed augmented-spherical wave band structure calculations, showing that $\mathrm{A}$ and $\mathrm{B}$ excitons are due to the smallest direct interband transitions at the $\mathrm{K}$ point in the Brillouin zone $[72,73]$; this result has important implications in few layer TMD optical properties (see below).

The thinnest 2D crystal that Frindt reported was about $15 \AA$ thick, which corresponds to bi-layers, and in the same manuscript, the possibility of reaching monolayers was also discussed [68]. Twenty years later, in 1986, Joensen, Frindt and Morrison published a manuscript entitled "Single Layer $\mathrm{MoS}_{2}$ " in which monolayers of $\mathrm{MoS}_{2}$ suspended in solution were obtained by lithium intercalation phases reacting with water [69]. When the solution was dried, the $\mathrm{MoS}_{2}$ monolayers rearrange themselves in a random way, according to X-Ray diffraction analysis. This early experiment indicated that it is possible to study different stacking orders of chemically exfoliated monolayers using different TMDs, thereby revealing fascinating electronic and thermal properties (see below).

In 2010, Mak, et al. confirmed the existence of a new direct band gap material from monolayers of trigonal prismatic $\mathrm{MoS}_{2}$ [60], and opened up the possibility of fabricating novel 2-D direct band gap materials out of semiconducting TMDs such as $\mathrm{WS}_{2}, \mathrm{WSe}_{2}$, $\mathrm{MoSe}_{2}$. These atomically thin layers could find applications in novel electronic and optical devices. Mak and colleagues produced thin $\mathrm{MoS}_{2}$ films ranging from 1 to 6 molecular layers (around $75 \AA$ thick) using the "Scotch tape" method and they characterized these samples using optical absorption, photoluminescence and photoconductivity [60]. The authors found that monolayered $\mathrm{MoS}_{2}$ exhibits an intense photoluminescence $(\mathrm{PL})$ signal around $1.84 \mathrm{eV}(673.8 \mathrm{~nm})$ that is related to the smallest 
direct gap transition at the $\mathrm{K}$ point in the Brillouin zone (A exciton), which is greatly quenched in the bi- and few-layered systems of $\mathrm{MoS}_{2}$; for films with more than one layer, the indirect band gap dominates. In particular, the indirect band gap $(1.29 \mathrm{eV}$ for the bulk which goes from the $\Gamma$ point to an intermediate point between $\Gamma$ and $\mathrm{K}$ ), increases as the number of layers decrease until becoming larger $(c a .1 .9 \mathrm{eV})$ than the direct band gap $(1.84 \mathrm{eV})$. The other direct transition at the $\mathrm{K}$ point (exciton B) is clearly identified at $c a$. $2 \mathrm{eV}(620 \mathrm{~nm})$ in the optical absorption spectra; this transition is mainly due to two factors: spin-orbit splitting at the $\mathrm{K}$ point and an interlayer interaction. The above results were subsequently confirmed by other groups in $\mathrm{MoS}_{2}$ samples prepared by mechanically exfoliated layers $[12,61]$. Other TMDs (e.g. $\mathrm{WS}_{2}, \mathrm{WSe}_{2}$ and $\mathrm{MoSe}_{2}$ ) with the same trigonal prismatic structure as $\mathrm{MoS}_{2}$, also exhibit direct band gaps for monolayers and indirect band gaps for bi-layer systems and for systems with more layers $[10,12,16,17$, 74].

Atomically thin crystals of $\mathrm{WS}_{2}$ have also been prepared using a CVD method, similar to that used by Tenne and co-workers for producing inorganic TMD Fullerenes and TMD nanotubes $[48,75]$. The $\mathrm{WS}_{2}$ monolayer crystals start growing with a triangular shape [76]. The PL signal for these monolayers was found at $c a .1 .94 \mathrm{eV}(637 \mathrm{~nm})$. Surprisingly, an extraordinary enhancement of the PL signal, not reported before, was found at the edges (or close to the edges) of these islands; so far, the explanation for this enhancement remains an open question. Moreover, in CVD grown crystals of $\mathrm{WS}_{2}$ that were mechanically exfoliated with adhesive tape, the PL peak is found at $c a .1 .96 \mathrm{eV}$ $(630 \mathrm{~nm})$ [77], and in large area CVD grown monolayers of $\mathrm{WS}_{2}$, the PL was reported to be at $c a .2 .02 \mathrm{eV}(613 \mathrm{~nm})$ [78]. This difference in PL wavelengths might be due to strain 
caused by the growing substrate used to synthesize the monolayers.

Raman spectroscopy constitutes a key characterization technique for identifying the number of layers in TMDs. For example, single layers of $\mathrm{WS}_{2}$ exhibits a unique double resonant process at a particular excitation wavelength which allows the precise identification of monolayers [79]. Therefore, double resonance Raman effects should also be present in other TMDs and could be used to identify the number of layers.

Density functional perturbation theory (DFPT) calculations have been carried out in order to study the vibrational modes, Raman and infrared spectra of several TMDs [80-83]. Table 1 shows the main Raman active modes of bulk and few layered TMDs: $A_{1 g}\left(A^{\prime}{ }_{1}\right), E_{2 g}^{1}\left(E^{\prime}\right)$ and $E_{1 g}\left(E^{\prime \prime}\right)$. As the layer thickness decreases, the $A_{1 g}$ mode intensity decreases and the $\mathrm{E}_{2 \mathrm{~g}}{ }^{1}$ mode increases. Further work needs to be carried out and understand the Raman signal from different TMDs. For example, the $A_{1 g}\left(A^{\prime}{ }_{1}\right)$ peak located at $c a .261 .1 \mathrm{~cm}^{-1}$ in $\mathrm{WSe}_{2}$ is not due to the $\mathrm{A}_{1 \mathrm{~g}}\left(\mathrm{~A}^{\prime}{ }_{1}\right)$, but rather to the $2 \mathrm{LA}(\mathrm{M})$ mode [84] (see Table 1).

Table 1 Experimental and DFPT calculated Raman active modes of different TMDs in bulk and few layered systems. The "*”" in $\mathrm{WSe}_{2}$ indicates that this mode is in fact the $2 \mathrm{LA}(\mathrm{M})$ mode.

\begin{tabular}{|c|c|c|c|c|c|c|}
\hline Structure/mode & $\begin{array}{l}\mathrm{A}_{1 \mathrm{~g}} \\
\left(\mathrm{~A}^{\prime}{ }_{1}\right) \mathrm{cm}^{-1} \\
(\mathrm{Exp})\end{array}$ & $\begin{array}{l}\mathrm{A}^{\prime}{ }_{1} \mathrm{~cm}^{-1} \\
(\mathrm{DFPT})\end{array}$ & $\begin{array}{l}\mathrm{E}_{2 \mathrm{~g}} \\
\left(\mathrm{E}^{\prime}\right) \mathrm{cm}^{-1} \\
(\mathrm{Exp})\end{array}$ & $\begin{array}{l}E^{\prime} \mathrm{cm}^{-1} \\
\text { (DFPT) }\end{array}$ & $\begin{array}{l}\mathrm{E}_{1 \mathrm{~g}}\left(\mathrm{E}^{\prime \prime}\right) \mathrm{cm}^{-1} \\
(\mathrm{EXP})\end{array}$ & $\begin{array}{l}\mathrm{E}^{\prime \prime} \mathrm{cm}^{-} \\
\text {(DFPT) }\end{array}$ \\
\hline \multirow{3}{*}{$\mathrm{MoS}_{2}$} & $408.3[82]$ & 402.7 & $383[82]$ & 383.6 & $286[82]$ & 284 \\
\hline & $409[80]$ & & $383[80]$ & & $287[80]$ & \\
\hline & $403.4[85]$ & & $384.6[85]$ & & & \\
\hline \multirow[t]{2}{*}{$\mathrm{MoSe}_{2}$} & $242[82]$ & 240.3 & $286[82]$ & 285.3 & $168[82]$ & 167.6 \\
\hline & $243[86]$ & & $287.2[86]$ & & & \\
\hline$\alpha-\mathrm{MoTe}_{2}$ & $174[82]$ & 173.7 & $234[82]$ & 238.7 & $116.8[82]$ & 119.7 \\
\hline \multirow[t]{3}{*}{$\mathrm{WS}_{2}$} & $423[82]$ & 418.7 & $357[82]$ & 359.2 & & 298.5 \\
\hline & $419[78]$ & & $358[78]$ & & & \\
\hline & $417.5[76]$ & & $356[76]$ & & & \\
\hline \multirow[t]{2}{*}{$\mathrm{WSe}_{2}$} & $251[82]$ & 250.2 & $247[82]$ & 249.3 & $186[82]$ & 175.7 \\
\hline & $253[81]$ & & $250[81]$ & & $178[81]$ & \\
\hline
\end{tabular}


$261.1[83]^{*}$

$251[84]$
249.1[83]

$248[84]$

$180.1[83]$

$\alpha-\mathrm{WTe}_{2}$

181.4

201.8

125.2

Although semiconducting TMDs share similarities regarding the presence of a direct band gap at the $\mathrm{K}$ point for monolayers of $\mathrm{MoS}_{2}$, and possess an indirect band gap in the bulk, there are particular unique differences that might be useful for applications in few layered systems. For example, in few layer exfoliated $\mathrm{MoSe}_{2}$ the indirect and direct band gaps are very close in energy, and it has been found that there is a PL enhancement with increasing temperature [87]; the direct band gap of monolayer $\mathrm{MoSe}_{2}$ according to PL measurements is around $1.55 \mathrm{eV}$, and it quenches as the number of layers increase. However, for 9 layers of $\mathrm{MoSe}_{2}$ the PL intensity increases as temperature increases up to $423 \mathrm{~K}$ [87]. This effect is the result of the closeness of the direct and indirect band gap energies in few layered systems (not present in $\mathrm{MoS}_{2}$ or $\mathrm{WS}_{2}$ ), and in certain TMD systems. High temperatures make the TMD layers decouple from their neighboring layers via an interlayer thermal expansion [87].

It turns out that the PL signal in $\mathrm{MoS}_{2}$ and $\mathrm{MoSe}_{2}$ could be enhanced by the physisorption of water and $\mathrm{O}_{2}$, due to the $n$-type unintentional doping which has been attributed to the presence of defects and substrate interactions [88-90], but the real reason for this doping has not been convincingly addressed so far. In this case, it has also been proposed that the physisorbed molecules deplete the electrons of $n$-type TMDs decreasing the electrostatic screening, thus creating a new radiative exciton recombination channel which helps enhancing the PL signal. It was also reported that for $\mathrm{WSe}_{2}$, the effect is just the opposite due to the $p$-type doping of this TMD material [91]. So far, controlling the $n$-type and $p$-type doping of TMDs remains a challenge, but this 
idea should be developed further into a novel line of research, thus widening the richness of few-layered TMDs.

Though most of the research thus far has been focused on semiconducting TMDs, it is worth mentioning that there are other TMD materials with metallic behavior such as: the octahedral metastable phases of $\mathrm{MoS}_{2}$ and $\mathrm{WS}_{2}$ [92-96] and the trigonal prismatic forms of $\mathrm{NbS}_{2}, \mathrm{NbSe}_{2}, \mathrm{TaS}_{2}$ and $\mathrm{TaSe}_{2}$, which besides being metallic are superconductors at low temperature, and reveal charge density wave effects (CDW), except for $\mathrm{NbS}_{2}$ systems [97-100] . Exploring the role of thickness in CDW behavior in these metallic TMDs also opens an alternative research line which should be developed further.

\subsection{Semiconducting monolayer and few layered TMDs}

The first early approaches to calculate the band structure of bulk $\mathrm{MoS}_{2}, \mathrm{MoSe}_{2}, \mathrm{WS}_{2}$ and $\mathrm{WSe}_{2}$, involved the use of semi-empirical tight binding methods, but the presence of the direct band gap at the $\mathrm{K}$ point in the Brillouin zone was not emphasized at that time $[101,102]$. In the book edited by Wieting and Schlüter, it reviews the different methods for calculating the electronic properties of TMDs that were used until the late seventies, but bulk crystals were considered at that time [103]. The implementation of first principles methods (ab-initio) using density functional theory (DFT) made possible the identification of the direct band gap at the $\mathrm{K}$ point in semiconducting monolayers of TMDs [104-115]. However, it is well known that in DFT, the electronic band gap is underestimated, and in order to obtain a more realistic approach, a GW approximation considering excitonic effects (solving the Bethe-Salpeter equation) and spin-orbit coupling is required $[106,114,116-119]$. In fact, it is a "lucky" coincidence that DFT 
calculations yield good values for the optical direct band gap. Therefore, DFT provides a reasonable first approximation, widely used, to understand some of the electronic and optical properties of different semiconducting TMDs.

For bi- and few-layers of semiconducting TMDs, the indirect band gap at the intermediate point between the path $\Gamma-\mathrm{K}$ dominates the electronic behavior of the 2-D material. Nevertheless, it is worth pointing out that for every layer stacked on top another layer, the electronic properties change drastically, and new stacked systems could be created. In this context, theoretical studies of few layered semiconducting TMDs involving twisting of stacked layers as well as the introduction of defects will be discussed below. Regarding bi-layers of TMDs, it has been demonstrated theoretically using DFT under the local density approximation (LDA) that the indirect band gap dominates their electronic behaviors. These calculations reveal how the direct and indirect band gaps change in bi-layer TMDs in comparison with the monolayer and the bulk; the lattice parameters are also shown in Ref. [109]. When considering spin-orbit coupling in ab-initio calculations, a band splitting at the $\mathrm{K}$ point appears in going from one layer to the bulk [117-119]. This is confirmed by optical absorption experiments in which the excitons A and B are identified, as discussed above. The spin-orbit splitting at the $\mathrm{K}$ point varies depending on the TMD system. For $\mathrm{MoS}_{2}$ the value corresponds to $0.15 \mathrm{eV}$, for $\mathrm{MoSe}_{2} 0.18 \mathrm{eV}$, for $\mathrm{WS}_{2} 0.43 \mathrm{eV}$ and for $\mathrm{WSe}_{2} 0.46 \mathrm{eV}$ [117-119]. In addition, the splitting of the valence band at the $\mathrm{K}$ point is also due to interlayer interactions and plays a role in few-layered systems. The breaking of inversion symmetry in TMDs and spin-orbit coupling lead to valleytronic effects which might be used in future optoelectronic applications [117, 120-123]. 


\section{Synthesis methods of TMDs: clusters, ribbons and}

\section{monolayers}

As mentioned above, mono- and few-layer TMDs reveal fascinating properties different from their bulk counterparts. In this context, the synthesis of monocrystalline layers of atomically thin TMDs are crucial for various applications. Up to now, various methods have been developed to obtain mono- and few-layer TMDs. All of them can be widely classified into two categories: "top-down routes", and "bottom-up routes". In this section, we summarize the state-of-the-art progress related to the synthesis techniques used to produce TMD atomic layers.

\subsection{Top-down routes}

"Top-down routes" denote those methods based on exfoliating bulk TMD crystals into atomically thin layers. Similar to graphite, the interaction between adjacent layers in bulk TMDs is dominated by the Van der Waals coupling, which is much weaker than the in-plane atomic bonds in TMDs. Therefore, if one can use physical (e.g., mechanical exfoliation [60,68, 89, 122, 124, 125]) or chemical (solvent exfoliation [126-129], Lithium-intercalation $[69,130-132])$ ways to weaken the Van der Waals forces in bulk TMD crystals, then few- or even single-layer materials could be obtained. Some examples of demonstrated "Top-down routes" are summarized in Figure 2. There are mainly two kinds of methods used for exfoliating bulk TMDs: one is mechanical exfoliation, and another is chemical exfoliation, as discussed below. 


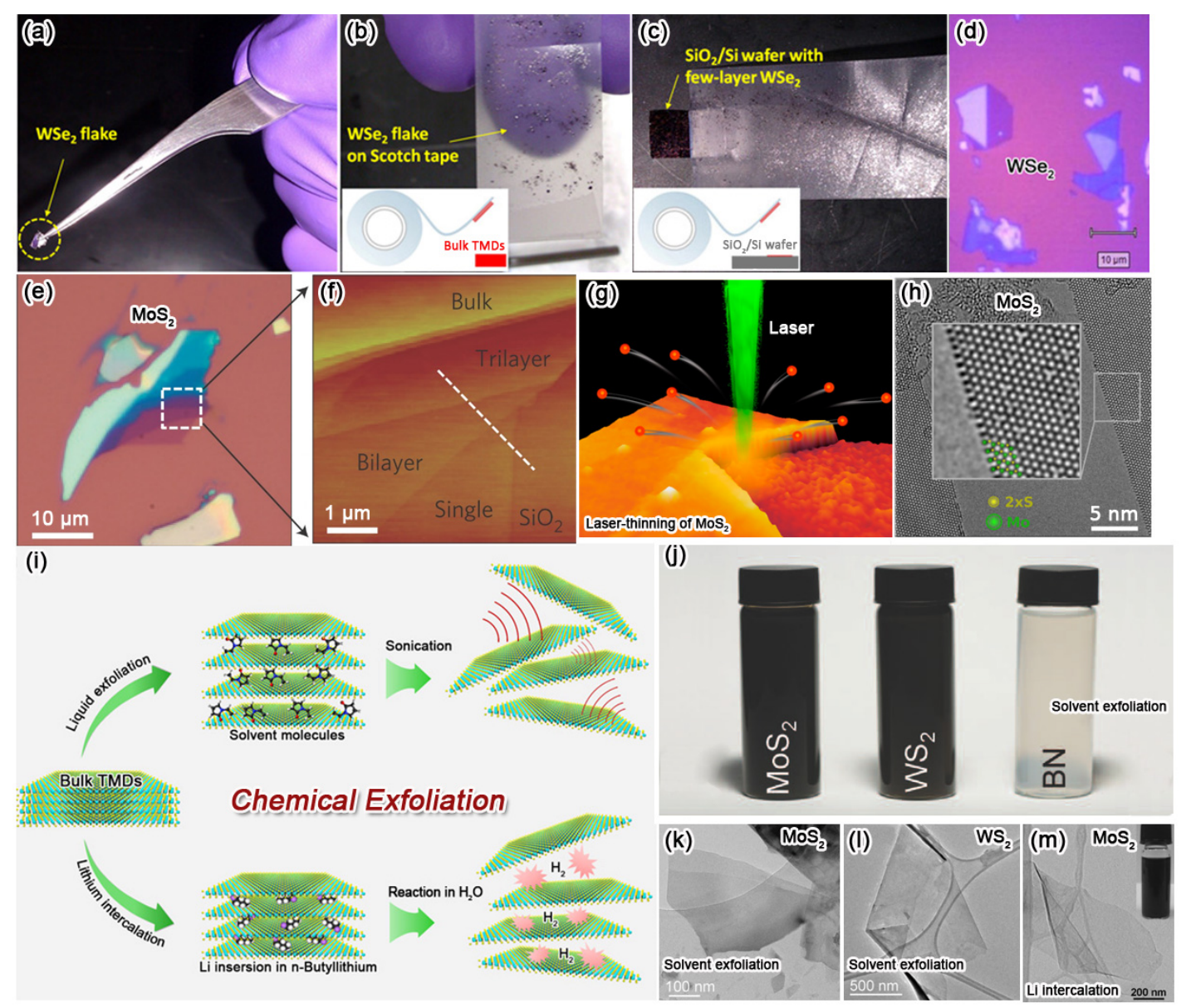

Figure 2 Top-down routes used for obtaining atomically thin TMDs. (a-c) Mechanical exfoliation method used for obtaining few-layer TMD sheets. The insets in (b) and (c) correspond to schemes for "Scotch tape" peeling steps; (d) Optical micrograph of as-exfoliated $\mathrm{WSe}_{2}$ flakes. (Images are kindly provided by Simin Feng and Jorge L. Castro Torres in the Terrones group); (e) Optical micrograph of a mechanically exfoliated $\mathrm{MoS}_{2}$ sample and (f) corresponding atomic force micrograph (AFM) of the highlighted area. Obvious contrast differences can be found in the optical micrograph of TMD sheets exhibiting different number of layers. Reprinted with permission from Ref. [122]. Copyrights 2013 Nature Publishing Group; (g) Laser-thinning techniques can be used to obtain single-layer $\mathrm{MoS}_{2}$ sheets from multi-layer systems. Reprinted with permission from Ref. [133]. Copyrights 2012 American Chemical Society; (h) An aberration-corrected high-resolution transmission electron microscopy (AC-HRTEM) image of monolayer $\mathrm{MoS}_{2}$. Reprinted with permission from Ref. [134]. Copyrights 2012 American Physical Society; (i) Chemical exfoliation method used for obtaining few-layer TMD sheets. Liquid (e.g. solvent[126]) or lithium[131] can be intercalated into the interlayer spacing of TMDs, and finally exfoliated into few- or single-layer sheets after sonication or chemical reactions (e.g. $\mathrm{H}_{2}$ generation by immersing the Li-intercalated TMDs into water); (j) Photographs of dispersions of $\mathrm{MoS}_{2}$ (in NMP), $\mathrm{WS}_{2}$ (in NMP), and BN (in IPA). Here NMP and IPA denote N-methyl-pyrrolidone and isopropanol, respectively. 
The corresponding TEM images of liquid exfoliated TMD sheets are shown in (k) $\mathrm{MoS}_{2}$ and (I) WS $\mathrm{WS}_{2}$. Reprinted with permission from Ref. [126]. Copyrights 2011 American Association for the Advancement of Science (AAAS), and (m) Typical TEM image of $\mathrm{MoS}_{2}$ nanosheets obtained by an electrochemical lithiation-exfoliation process. Reprinted with permission from Ref. [131]. Copyrights 2011 Wiley-VCH Verlag GmbH \& Co. KGaA, Weinheim.

\subsubsection{Mechanical exfoliation}

Mechanical exfoliation, or the so-called "Scotch-tape method", has been widely used for obtaining mono-layer graphene sheets by repeatedly peeling bulk graphite with adhesive tape [135]. As described above, in 1966, Frindt [68] used this method to peel bulk $\mathrm{MoS}_{2}$ into thin sheets exhibiting several molecular layers ( $\sim 3.5 \mathrm{~nm}$ thick). Actually, it is also possible to find single-layer TMDs just by rubbing the layered crystals against another solid surface (e.g., a $\mathrm{SiO}_{2} / \mathrm{Si}$ wafer), similar to "drawing by chalk on a blackboard" [124]. In addition, due to the high-transparency of atomically thin TMDs, their visibility under an optical microscope depends highly on the substrate used for supporting the samples. It was found that an oxidized $\mathrm{Si}$ wafer $\left(\right.$ i.e. $\mathrm{SiO}_{2} / \mathrm{Si}$ ) can serve as a better substrate material than mica due to the nice phase contrast between the TMD-covered area and the empty part on the $\mathrm{SiO}_{2} / \mathrm{Si}$ substrate [124]. A modified process for mechanically exfoliating TMDs is show in Figure 2a-d. Firstly, a piece of a bulk TMD flake (e.g., the $\mathrm{WSe}_{2}$ flake shown in Figure 2a) was adhered onto a piece of Scotch tape. Subsequently, the tape with the $\mathrm{WSe}_{2}$ flake was folded and separated repeatedly for many times to get few-layer sheets (Figure 2b). Next, the tape with few-layer $\mathrm{WSe}_{2}$ sheets was adhered onto a clean $\mathrm{SiO}_{2} / \mathrm{Si}$ wafer with a $285 \mathrm{~nm}$ thick $\mathrm{SiO}_{2}$ layer (Figure 2c). Finally, the as-obtained few-layered $\mathrm{WSe}_{2}$ sample was characterized by different techniques (e.g. optical microscopy, Raman spectroscopy, atomic force microscopy) in 
order to identify the number of layers and to be able to explore their electrical, optical or chemical properties. A typical optical micrograph of an as-obtained few-layer $\mathrm{WSe}_{2}$ sample was shown in Figure 2d. An obvious contrast difference between the bulk and few-layer TMD sheets can be seen in optical micrographs when $\mathrm{SiO}_{2}(285 \mathrm{~nm}$ thick $) / \mathrm{Si}$ was used as a substrate (see Figure 2e-f). After performing image analysis, the number of layers of as-exfoliated $\mathrm{MoS}_{2}$ can be identified using optical micrographs [136]. However, most of the products from mechanical exfoliation are multi-layer TMD sheets instead of single-layers. In order to further thin multi-layer sheets down to monolayered TMDs, some physical thinning techniques (e.g., laser-thinning [133] shown in Figure 2g) can be used. In particular, the shape and size of the final monolayers of TMD sheets can be controlled by varying the laser power and scanning area [133]. Figure $2 \mathrm{~h}$ shows a typical aberration-corrected high-resolution transmission electron microscopy (AC-HRTEM) image of monolayered $\mathrm{MoS}_{2}$ obtained after mechanical exfoliation [134]. The atomic scale structure of $\mathrm{MoS}_{2}$ can be clearly observed. Recently, Chen et al. have demonstrated that mechanical exfoliation of TMD alloys $\left(\mathrm{Mo}_{1-\mathrm{x}} \mathrm{W}_{\mathrm{x}} \mathrm{S}_{2}\right)$ can yield monolayer alloys with tunable band gaps by altering the alloy composition [137]. Among different methods, mechanical exfoliation appears to be the easiest way to obtain highly crystalline few-layer TMD sheets. More importantly, exfoliated TMD sheets can keep the inherent properties of the material, and are ideal for demonstrating their applications in the fabrication of electrical devices [125, 138], ultrasensitive photodetectors [139], photovoltaic devices [140], chemical vapor sensing [141], etc. 


\subsubsection{Chemical exfoliation}

Although mechanical exfoliation can generate high-quality single or few-layer TMD sheets, the method is not suitable for scale-up applications. In this context, chemical exfoliation constitutes an alternative way to exfoliate bulk TMD flakes into few- or single-layer sheets on a large scale by "intercalation-exfoliation" processes. Up to now, there are two main routes for chemically exfoliating TMD flakes (shown in Figure 2i). One is liquid exfoliation, and the other is lithium intercalation, followed by exfoliation. A recent review article summarizes the state-of-the-art progress on liquid exfoliation of different layered materials [74]. An effective strategy for exfoliating TMDs consists of exposing the bulk materials under ultrasonic waves in a suitable solvent [126]. Theoretical calculations have shown that if the surface energy of an as-used solvent is similar to that of the layered materials, the driving force for re-aggregation of exfoliated sheets can be overcome to form stable suspensions in solvents [142]. Different layered compounds including $\mathrm{MoS}_{2}, \mathrm{WS}_{2}, \mathrm{MoSe}_{2}, \mathrm{MoTe}_{2}, \mathrm{TaSe}_{2}, \mathrm{NbSe}_{2}, \mathrm{NiTe}_{2}, \mathrm{BN}$ and $\mathrm{Bi}_{2} \mathrm{Te}_{3}$, have been successfully exfoliated into few-layer sheets [126]. Typical photographs and TEM images of as-exfoliated $\mathrm{MoS}_{2}$ and $\mathrm{WS}_{2}$ are shown in Figure 2j and Figure 2k-1, respectively. N-methyl-pyrrolidone (NMP) has been proved to be an ideal solvent for exfoliating $\mathrm{MoS}_{2}$ and $\mathrm{WS}_{2}$ efficiently [126]. Furthermore, by mixing two "poor" solvents (e.g. water, ethanol), the dispersability of TMDs in the solvent mixture will work much better than when using a single solvent for exfoliating TMDs [128]. This method appears to be ideal for the low-cost and scalable production of few-layer TMD sheets.

Lithium intercalation could also be used to exfoliate TMDs by violent reactions involving Li intercalates and water. In 1975, Dines et al. successfully synthesized the 
stoichiometric Li-intercalation complexes in diverse TMDs using $n$-butyllithium/hexane [130]. It is well known that $\mathrm{Li}$ will react with water violently to generate hydrogen gas, which actually can be used to separate bulk TMDs into few-layer or monolayer nanosheets with the assistance of ultrasonication. In 1986, Joensen et al. exfoliated $\mathrm{MoS}_{2}$ into monolayers based on this concept [69]. However, the intercalation of Li into TMDs layers is time-consuming (at least 48 hours in a dry box) and not well-controlled [69]. An alternative, faster and more controllable route for $\mathrm{Li}$ intercalation consists of using an electrochemical cell with $\mathrm{Li}$ foil as an anode and TMD-containing a cathode. The intercalation process can be completed after several hours. More importantly, the intercalation of Li into bulk TMDs can be monitored and well controlled during the discharge process [131]. After reacting this intercalated material with water, mono- or few-layered TMDs nanosheets can be obtained. A typical TEM image of an as-exfoliated $\mathrm{MoS}_{2}$ nanosheet is shown in Figure $2 \mathrm{~m}$.

The obvious advantage of chemical exfoliation methods is their potential for mass production of few-layered TMD nanosheets. Unfortunately, the sonication process widely used in chemical exfoliation is detrimental to the exfoliated materials when obtaining few-layer TMD sheets with large lateral size (e.g. more than square microns or even $\mathrm{cm}^{2}$ ). Moreover, the degree of crystallinity, and the electrical properties such as the carrier mobility of as-exfoliated TMD sheets are different from their bulk crystalline counterparts [127, 143]. A novel approach using gentler Brønsted acid based intercalation seems promising in overcoming these problems. This technique was used to reversibly intercalate graphite and h-BN under solvent free conditions. During this intercalation process, the bulk layered material is subjected to acid treatment followed by heating to 
dryness, which is a crucial step leading to intercalation [144]. Nevertheless, because of their attractive physical/chemical properties and scale-up potential, TMD sheets obtained from chemical exfoliation may find applications in diverse areas such as hydrogen evolution [129, 145-148], biomolecule detection [149-151], photoelectrochemical cells $[132,152]$, etc.

\subsection{Bottom-up routes}

"Bottom-up routes" denote those methods based on the directed or the self-assembly of precursor molecules able to synthesize few- or single-layer TMD structures. So far, chemical vapor deposition (CVD) is the most widely used method for synthesizing TMD sheets $[78,153-159]$, triangular clusters $[76,79,160-164]$ or nanoribbons $[165,166]$. In this section, we summarize some progress achieved in the CVD synthesis of atomically thin TMD layers. Other "bottom-up" methods, such as physical vapor transport [167], wet chemical approaches [168], will also be introduced.

\subsubsection{Chemical vapor deposition}

CVD is a very important technique for synthesizing various inorganic nanomaterials (e.g. carbon nanotubes $[169,170]$, graphene $[171,172]$, and BN nanostructures $[173$, 174]). It is different from PVD techniques (e.g. electron-beam deposition, sputtering deposition) since a chemical reaction occurs at relatively high temperatures. In a typical CVD process for growing atomically thin TMDs, a substrate (usually a $\mathrm{SiO}_{2} / \mathrm{Si}$ wafer or sapphire) is coated with a thin layer of a transition metal or metal oxide (e.g. Mo, MoOx) 
using PVD (e.g. electron-beam deposition). Subsequently, the substrate is then exposed to a chalcogen atmosphere (e.g. sulfur powder $[76,153])$ at high temperature (e.g. $750{ }^{\circ} \mathrm{C}$ [153]), and finally a TMD thin layer structures is formed. Figures 3b-c show a typical optical micrograph and the corresponding Raman $\mathrm{E}_{2 \mathrm{~g}}^{1}$ mapping of as-synthesized $\mathrm{MoS}_{2}$ atomic layers grown on a $\mathrm{SiO}_{2} / \mathrm{Si}$ substrate. The number of layers in $\mathrm{MoS}_{2}$ are marked in Figure $3 \mathrm{c}$ [153]. Due to the high melting point of tungsten $\left(3422{ }^{\circ} \mathrm{C}\right)$, it is not easy to coat substrates with the pure $\mathrm{W}$ metal by electron-beam deposition. However, tungsten trioxide $\left(\mathrm{WO}_{3}\right)$, exhibiting a melting point of $1473{ }^{\circ} \mathrm{C}$, can be used as an alternative choice. Figure 3d depicts a scheme for growing $\mathrm{WS}_{2}$ atomic layers by sulfurizing thin $\mathrm{WO}_{3}$ coatings (5-10 $\AA$ thick) on $\mathrm{SiO}_{2} / \mathrm{Si}$ substrates at $\sim 800{ }^{\circ} \mathrm{C}$. A typical SEM image and the corresponding photoluminescence (PL) mappings of as-grown $\mathrm{WS}_{2}$ triangular clusters are shown in Figure 3e and f, respectively. It is found that the PL intensity weakens significantly by increasing the number of $\mathrm{WS}_{2}$ layers due to the transition from a direct band gap semiconductor for a monolayer to an indirect gap semiconductor, as it usually observed in multilayer semiconducting TMD systems. Interestingly, the structure and chemical composition of the edges also have a unique effect regarding the PL intensity enhancement. As shown in Figure 3f, the edges of a $\mathrm{WS}_{2}$ monolayer triangular cluster display much stronger PL signals when compared to the central region of the triangular island [76]. In addition to the electron-beam deposition of transition metals or their oxides onto substrates, some other chemical precursors (e.g. $\left(\mathrm{NH}_{4}\right)_{2} \mathrm{MoS}_{4}$ [154]) can also be vaporized and then sulfurized in order to synthesize metal disulfide nanosheets (see Figure 3g). Moreover, transition metal oxide powders (e.g. $\mathrm{WO}_{3}, \mathrm{MoO}_{3}$ ) can also be used as solid precursors (Figure $3 \mathrm{~h}$ ) in the presence of sulfur vapor. The obvious advantage of 
this technique is that it does not require the expensive electron-beam deposition process needed in the methods shown in Figure 3a and d. It is also noteworthy that in order to initiate the growth of atomically thin TMD layers instead of particles, nucleation sites on the substrate are necessary. In this context, perylene-3,4,9,10-tetracarboxylic acid tetrapotassium salt (PTAS) has been demonstrated to be an effective seed material for the growth of $\mathrm{MoS}_{2}$ and $\mathrm{WS}_{2}$ monolayers, possibly because PTAS can facilitate the adsorption of precursor molecules, thus initiating the heterogeneous nucleation process [164]. Uniform PL intensities in as-synthesized $\mathrm{WS}_{2}$ triangular monolayers can be found in Figure 3i [164]. This is different from the results shown in Figure 3f, where the edge sites demonstrate a much enhanced PL than the center parts [76]. Further investigations are still needed to clarify the chemical state difference between the edges and center parts of as-grown TMD thin layers. CVD has also proven to be an excellent method to synthesize lateral as well as vertical TMD heterostructures with interesting electronic properties. There has been a lot of effort in this direction, particularly in trying to understand inter-layer coupling between individual TMD layers in a heterostructure. These hybrid materials show great potential in photovoltaic and optoelectronic applications [175-177].

CVD is a versatile synthesis method and can also be used to synthesize TMD nanoribbons. For example, when using single-walled carbon nanotubes (SWCNTs) as templates, $\mathrm{MoS}_{2}$ [165] and $\mathrm{WS}_{2}$ [166] nanoribbons have been successfully synthesized inside the tubes. In a typical synthesis experiment, the transition metal precursor (e.g. $\mathrm{H}_{3} \mathrm{PMo}_{12} \mathrm{O}_{40}$ for Mo [165], $\mathrm{H}_{3} \mathrm{PW}_{12} \mathrm{O}_{40}$ for $\mathrm{W}$ [166]) is filled into the hollow core of CNTs using sonication and reflux. The filled CNTs are then separated from the precursor 
aqueous solution by filtration and subsequent rinsing in deionized water. The resulting sample is then dried and used to react with a chalcogen precursor (e.g. $\mathrm{H}_{2} \mathrm{~S}$ ) in a $\mathrm{H}_{2}$ rich atmosphere at $800{ }^{\circ} \mathrm{C}$ for $2 \mathrm{~h}$, and finally TMD nanoribbons form inside the CNTs. Interestingly, the size of the final TMD nanoribbons strongly depends on the CNT diameter. The widths of as-synthesized $\mathrm{MoS}_{2}$ and $\mathrm{WS}_{2}$ nanoribbons have been reported in the range of 1 and $4 \mathrm{~nm}$ when SWCNTs and DWCNTs are used as templates [165, $166]$.
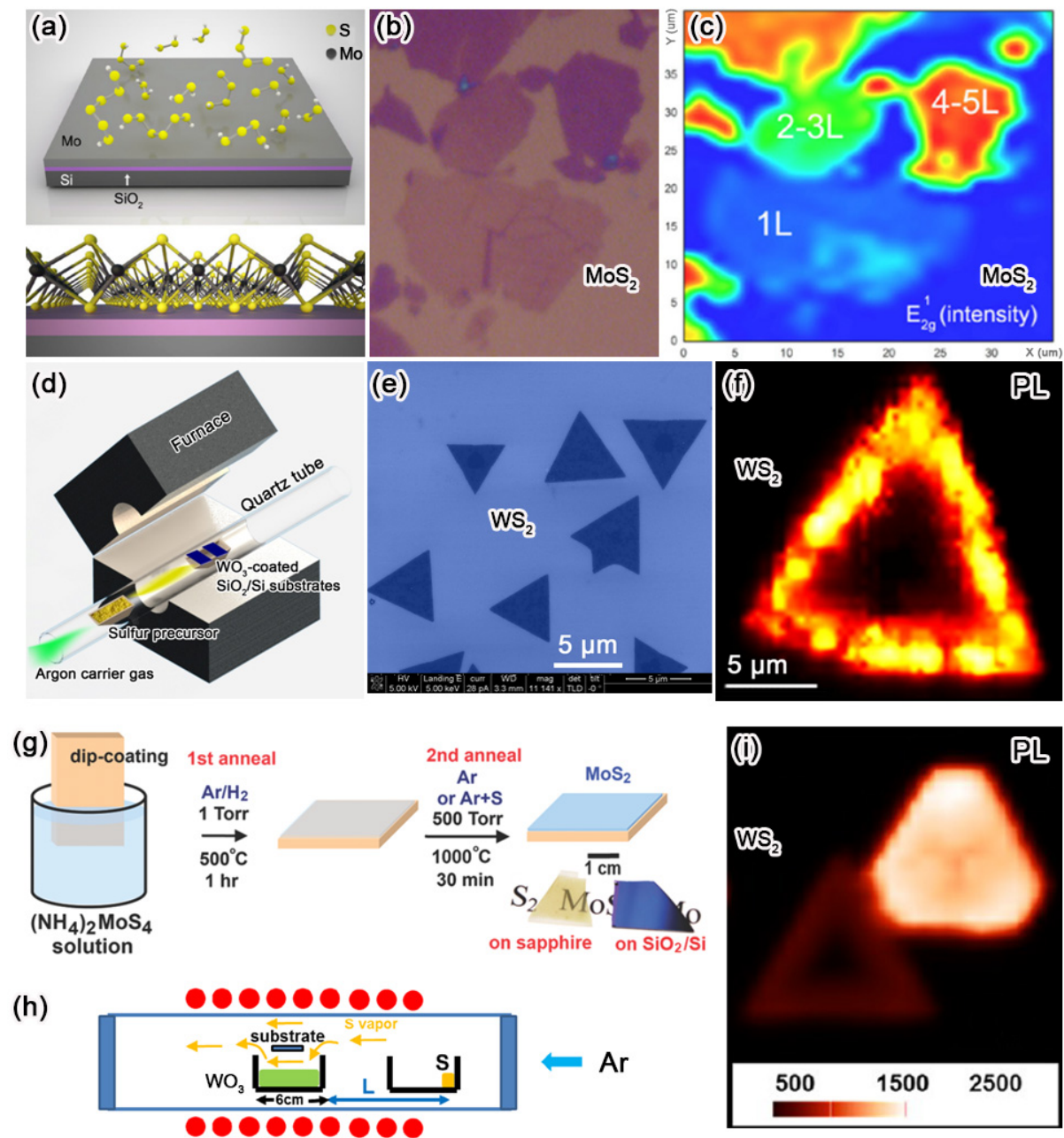

(h)

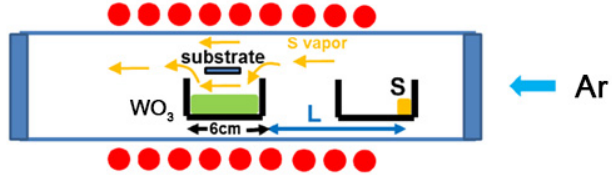

Figure 3 Schematics summarizing different chemical vapor deposition methods for obtaining atomically thin TMDs, including characterization of the materials. (a) Growth of $\mathrm{MoS}_{2}$ atomic layers, A Mo thin film (1-5 nm) was pre-deposited onto the $\mathrm{SiO}_{2} / \mathrm{Si}$ substrate by e-beam evaporator, then sulfur vapor is introduced to react with Mo at $750{ }^{\circ} \mathrm{C}$ in order to form $\mathrm{MoS}_{2}$ atomic layers; (b) Typical optical 
micrograph of as-grown $\mathrm{MoS}_{2}$ material, and its (c) corresponding Raman mapping of the Raman active $\mathrm{E}_{2 \mathrm{~g}}^{1}$ mode. The number of $\mathrm{MoS}_{2}$ layers is marked in the image. Reprinted with permission from Ref. [153]. Copyrights 2012 Wiley-VCH Verlag GmbH \& Co. KGaA, Weinheim; (d) Schematics showing the experimental set up used for growing $\mathrm{WS}_{2}$ atomic layers. In particular, a $\mathrm{WO}_{3}$ thin film and sulfur powders were used as $\mathrm{W}$ and $\mathrm{S}$ precursors, respectively; (e) Typical SEM images of as-synthesized triangular monolayers of $\mathrm{WS}_{2}$ and (f) its corresponding photoluminescence $(\mathrm{PL})$ mapping recorded at room temperature. Reprinted with permission from Ref. [76]. Copyrights 2013 American Chemical Society; (g) Thermolysis of Mo and S precursors (e.g. $\left.\left(\mathrm{NH}_{4}\right)_{2} \mathrm{MoS}_{4}\right)$ used for the synthesis of $\mathrm{MoS}_{2}$ thin layers. The precursor was dip-coated onto different insulating substrates (e.g. $\mathrm{SiO}_{2} / \mathrm{Si}$, sapphire), and then annealed using a two-step process. Reprinted with permission from Ref. [154]. Copyrights 2012 American Chemical Society; (h) Growth of monolayers of $\mathrm{WS}_{2}$ using $\mathrm{WO}_{3}$ and $\mathrm{S}$ as precursors; (i) PL mappings of as-synthesized $\mathrm{WS}_{2}$ triangles. It should be noted that nucleation sites (e.g. PTAS, perylene-3,4,9,10-tetracarboxylic acid tetrapotassium salt) are needed to initiate the $W^{2} S_{2}$ growth. Reprinted with permission from Ref. [164]. Copyrights 2013 American Chemical Society.

\subsubsection{Other methods}

Physical vapor transport ( PVT) is also an alternative to grow few layered TMD nanostructures $[167,178]$. When using TMD powder as a source material, a high optical quality monolayer TMD can be obtained via a vapor-solid (VS) growth mechanism [167]. In a typical run, $\mathrm{MoS}_{2}$ powder placed inside an alumina boat is located at the center of a horizontal quartz tube furnace; the pre-cleaned insulating substrates $\left(\mathrm{SiO}_{2} / \mathrm{Si}\right.$ wafer, sapphire or normal glass) is then placed in a downstream location in the low-temperature region $\left(\sim 650{ }^{\circ} \mathrm{C}\right.$ during growth). Subsequently, the quartz tube is heated up to $900{ }^{\circ} \mathrm{C}$ and a pressure of 20 mTorr is maintained under an Ar flow. After 15-20 min growth, triangular monolayers of $\mathrm{MoS}_{2}$ are obtained on the insulating substrates [167]. When compared with the CVD methods mentioned above, this PVD growth process appears to be simple, but only triangular clusters have been obtained with this method so far. In addition, the method has not yet been used to grow monolayers of $\mathrm{WS}_{2}$ or other 
atomically thin TMD films. Hydrothermal synthesis can also be used to grow TMD nanostructures via a "bottom-up" approach [168, 179]. For synthesizing $\mathrm{MoS}_{2}$ nanosheets, Mo precursors (e.g. $\left(\mathrm{NH}_{4}\right)_{6} \mathrm{Mo}_{7} \mathrm{O}_{24} \cdot 4 \mathrm{H}_{2} \mathrm{O}$ [179], $\mathrm{MoO}_{3}$ [168]) and $S$ precursor (elemental sulfur [179], potassium thiocyanate [168]) are added into deionized water in a Teflon-coated autoclave, and then react at a certain temperature (e.g. $150-180{ }^{\circ} \mathrm{C}$ ) for different periods of time (e.g., 24-48 h). The resulting product is then filtered, rinsed and dried. In this way, thin $\mathrm{MoS}_{2}$ nanosheets have been obtained. It is important to point out that the hydrothermal reaction offers an alternative scalable route for the synthesis of few-layered TMDs. However, the electrical and optical properties of as-synthesized products have not been studied in detail. Besides those mentioned above, many other methods, such as chemical synthesis using colloidal chemistry [180], have also been demonstrated. However, the wet chemical synthesis of TMD nanosheets is still in its infancy. Some challenges ahead include: (i) selection of appropriate metal and chalcogen precursors, (ii) careful control over metal oxidation states, and (iii) achieving high purity and yield.

\section{Raman spectroscopy of monolayer and few-layer TMDs}

Raman spectroscopy has become a key tool to study low-dimensional systems. This powerful and nondestructive technique has been widely used for decades to study layered TMDs in bulk [81, 181-185], carbon nanotubes [186] and fullerene-like structures [187]. More recently, the Raman response of atom-thick layers of TMDs has been studied as a function of the number of layers $[76,79,83,85,86,188-193]$, and excitation laser wavelengths $[79,85,194,195]$. Raman spectroscopy has also been used to provide 
insights about electron-phonon coupling interactions in TMDs [195, 196]. The sensitivity of the Raman response arises from changes in the (1) atomic restoring forces [188], (2) dielectric screening [188, 189], (3) electronic band structure [79], and (4) crystal symmetry [192]. In this section we summarize the most relevant vibrational Raman modes in mono- and few-layered TMDs.

\subsection{First-order Raman Modes in TMDs}

The thinnest film of $\mathrm{MX}_{2}$ (where $\mathrm{M}=[\mathrm{Mo}, \mathrm{W}]$ and $\mathrm{X}=[\mathrm{S}, \mathrm{Se}, \mathrm{Te}]$ ) is formed by stacking three elementary atomic layers; the central atomic layer of this stack contains positively charged metal atoms (M) with a 6-fold coordination symmetry, hexagonally packed between two trigonal atomic layers of negatively charged chalcogen atoms (X). Hence, the thinnest possible film of $\mathrm{MX}_{2}$ compounds could be represented as a $\mathrm{X}-\mathrm{M}-\mathrm{X}$ trilayer (TL). Although a $\mathrm{MX}_{2}$ single layer could belong to the symmetry space group P6m2 [189, 190, 192], the bulk phase (2H type) belongs to the P63/mmc space group [181, 189, 190, 192]. $\mathrm{MX}_{2}$ films containing few layers with an even (odd) number of TLs belong to the space group P3m1 (P6m2), respectively $[189,192]$. The primitive unit cell for bulk $\mathrm{MX}_{2}$, which contains six atoms, has 18 phonon branches corresponding to 6 acoustic and 12 optical phonon modes.

At the $\Gamma$ point of the Brillouin zone, the irreducible representation of the phonon modes can be described as [181]:

$\Gamma$ Bulk $=A 1 g \quad 2 A 2 u \quad B 1 u \quad 2 B 2 g \quad E 1 g \quad 2 E 1 u \quad E 2 u \quad 2 E 2 g$

Four of these modes are Raman-active $(E 2 g 1, A 1 g, E 1 g$ and $E 2 g 2)$ and two are 
IR-active $(A 2 u$ and $E 7 u)$. The $E$ modes are in-plane vibrations, while the $A$ modes are out-of-plane vibrations (along the z-axis). For a single-TL of $\mathrm{MX}_{2}$, the irreducible representation of the zone center phonons can be written as: $\Gamma 1 T L=A 1^{\prime} \quad E{ }^{\prime \prime}$ $2 A 2^{\prime \prime} 2 E^{\prime}$ [192], where the $A 1^{\prime}, E^{\prime \prime}$ and $E^{\prime}$ modes are the equivalents to the $A 7 g, E 1 g$ and $E 2 g 1$ modes of the bulk, respectively.

In general, the backscattering configuration is adopted to study single- and few-leyered $\mathrm{MX}_{2}$ films. In this geometry, the $E 1 g$ mode cannot be observed due to the Raman selection rules, and the $E 2 g 2$ mode is a low-frequency mode that requires special spectrometers with enhanced rejection of the Rayleigh scattered radiation to be observed [182, 192, 197, 198], will be shown in the next sections. For these reasons, many reports $[79,83,85,86,188-193]$ have focused on studying the $A 1 g$ and $E 2 g 1$ modes which can be easily probed by standard Raman spectrometers. In a recent study we investigated new Raman-active first order modes $\left(\mathrm{A}^{\prime}{ }_{1}^{2}\right.$ and $\left.\mathrm{A}_{1 \mathrm{~g}}{ }^{2}\right)$ in few layer semiconducting TMDs that are not observed in monolayer or bulk TMD. Polarized Raman spectroscopy as well as density functional perturbation theory (DFPT) were used to conduct detailed analysis in this direction [199].

\subsection{Dependence on the number of trilayers}




\subsubsection{E2g $1(\Gamma)$ and $A 1 g(\Gamma)$ phonon modes}

The thickness-dependent frequency of the $E 2 g 1(\Gamma)$ and $A 1 g(\Gamma)$ phonons have been studied for different TMDs, including $\mathrm{MoS}_{2}[188,192,195], \mathrm{WS}_{2}[79,191], \mathrm{MoSe}_{2}[86]$ and $\mathrm{WSe}_{2}[86,191,192]$. One can naively think that, when decreasing the number of TLs, the reduction of the van der Waals forces between TLs will decrease the effective restoring forces acting on the atoms, and thus the frequency of all the Raman-active phonon modes also decrease. In general, the above reasoning is valid for the out-of-plane A $1 g$ (Г) mode, mainly involving chalcogen atoms; this mode redshifts with decreasing number of TLs. However, a different trend (blue shift) has been observed for the E2g 1(Г), suggesting a hardening of this mode for a decreased number of TLs [79, 86, 188]. Molina-Sanchez and Wirtz [189] explained this anomalous behavior of the in-plane phonon mode $E 2 g 1(\Gamma)$ by analyzing each term of the real-space atomic force constant matrix. They found that due to a strong increase of the dielectric tensor components, the long-range Coulomb interactions are smaller for the bulk, when compared to a single-TL.

The frequency changes in both $A 1 g(\Gamma)$ and $E 2 g 1(\Gamma)$, as well as the difference between them, has been widely adopted $[79,83,85,86,188-193]$ as one of the methods to identify the number of TLs in few-TLs $\mathrm{MX}_{2}$ films. This behavior has been observed in both free-standing (suspended) and supported films (Figure 4a), and apparently does not depend on the excitation wavelength (Figure 4a-b). 

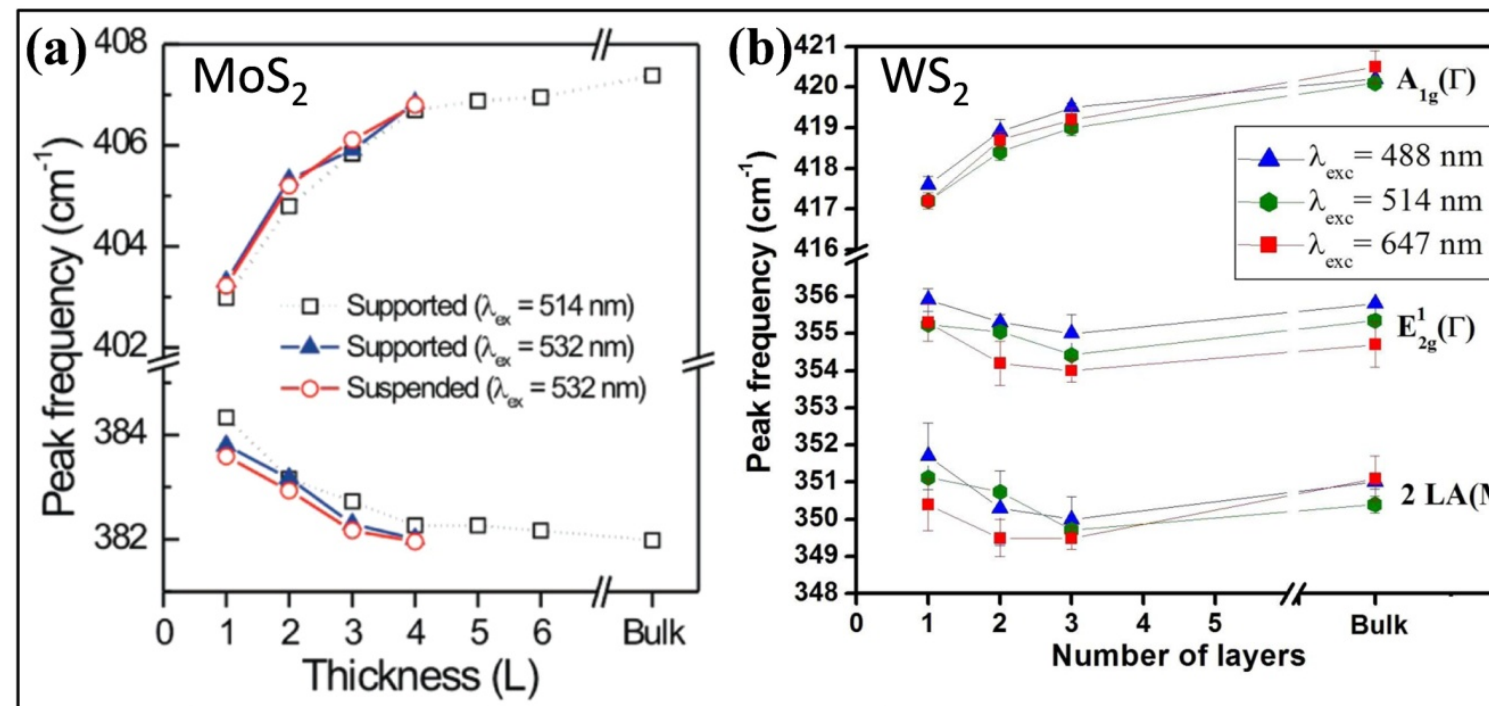

(c)

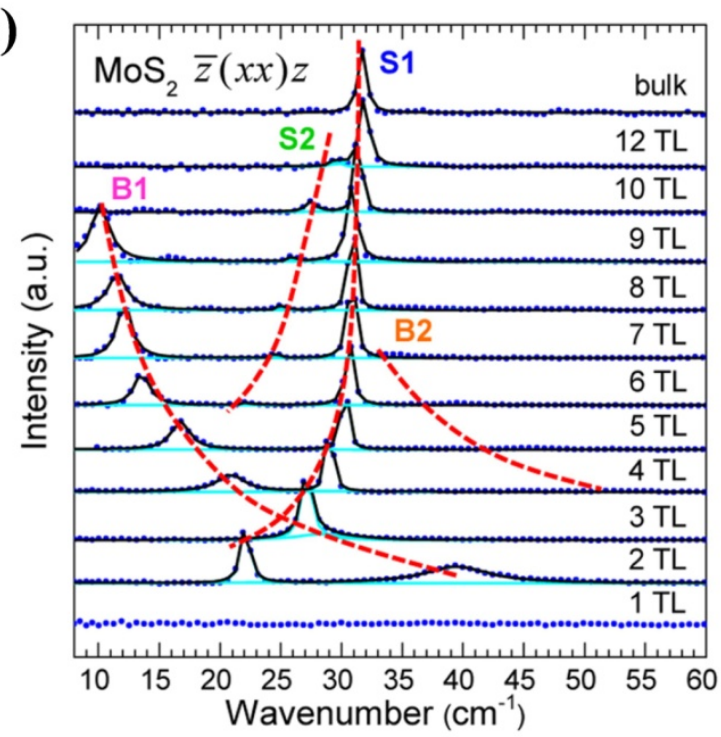

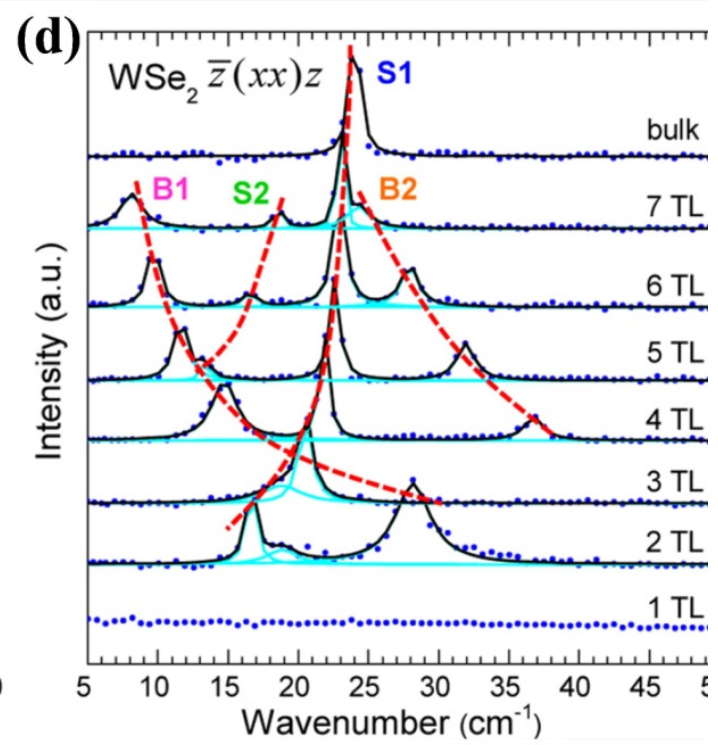
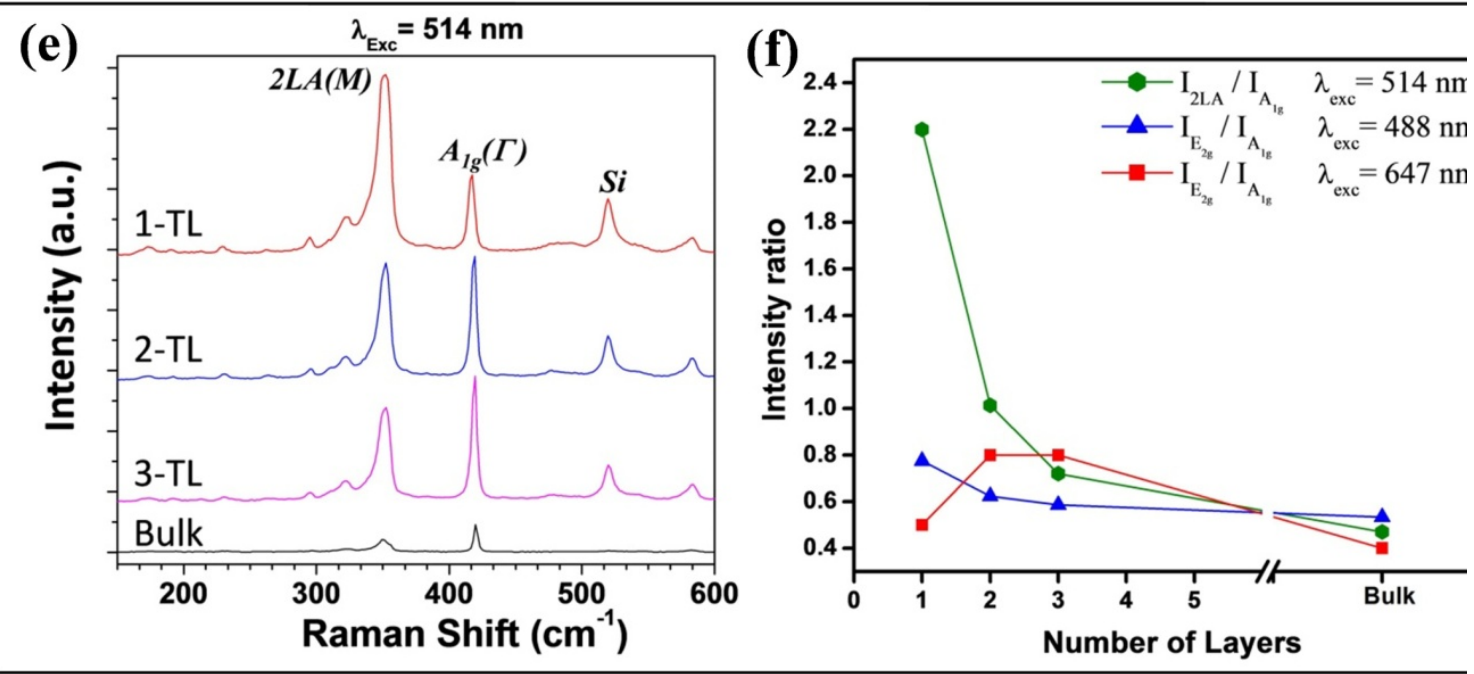
Figure 4 (a) Frequencies of the $E_{2 g}^{1}$ and $A_{1 g}$ Raman modes as a function of layer thickness for $\mathrm{MoS}_{2}$ samples supported on the substrate and suspended over holes. Reprinted with permission from Ref. [188]. Copyrights 2010 American Chemical Society. (b) Frequencies of $E_{2 g}^{1}, A_{1 g}$ and LA Raman modes as a function of layer thickness in $\mathrm{WS}_{2}$ samples using three different excitation wavelengths. Reprinted with permission from Ref. [79]. Copyrights 2013 Nature Publishing Group. (c) and (d) are the low-frequency Raman spectra evolutions as a function of trilayer (TL) number in (c) $\mathrm{MoS}_{2}$ and (d) $\mathrm{WSe}_{2}$, measured using $\bar{z}(x x) z$ polarization. Reprinted with permission from Ref. [192]. Copyrights 2013 American Chemical Society; S1, S2, B1 and B2 are the shear (S) and breathing (B) modes for this low-frequency range. (e) and (f) show the thickness dependence of the Raman peak intensities in $W_{2}$ few-layers films using $415 \mathrm{~nm}$ laser excitation wavelength and a resonant behavior is observed in single-TL. Reprinted with permission from Ref. [79]. Copyrights 2013 Nature Publishing Group.

\subsubsection{Interlayer breathing and shear modes}

Interlayer breathing and shear modes are commonly found in layered crystal structures.

In both modes, adjacent layers rigidly oscillate relative to each other. However, for the breathing mode, the oscillation amplitude is perpendicular to the layer plane (out-of-plane vibration), while in the shear mode, the oscillation amplitude lies in the layer plane (in-plane oscillation). For Bulk phases, these modes correspond to the $E 2 g 2$ (shear) and $B 2 g 2$ (breathing) phonons. Although the $B 2 g 2$ mode is optically inactive for the bulk, for few-TL films, the breathing modes become active [192].

Breathing and shear modes have very low frequencies (on the order of a few tens of wavenumbers) and are difficult to distinguish from the strong Rayleigh scattering background. Zhao et al. [192] recently studied these modes in few-TLs $\mathrm{MoS}_{2}$ and $\mathrm{WSe}_{2}$ films as a function of thickness, using a triple-grating Raman spectrometer, capable of filtering the side bands of the Rayleigh background, thereby reaching a detection limit of 
$\sim 5 \mathrm{~cm}^{-1}$. This group observed four low-frequency peaks associated with two shear modes (S1 and S2) and two breathing modes (B1 and B2). Interestingly, the frequencies of the shear modes (S1, S2) redshift as the number of TLs decreases, while the breathing modes (B1, B2) blueshift, as observed in Figure 4c-d. These modes are more sensitive to changes in the stacking order and number of TLs rather than the $A 1 g(\Gamma)$ and $E 2 g$ 1(Г) modes [192].

\subsection{Resonant Raman and second order modes}

Raman spectroscopy is useful not only to identify the number of layers, but also to probe details in the electronic band structure and phonon dispersion through resonant Raman scattering [200, 201]. A double Raman process involves [202-204]: (i) an initial electronic transition induced by the absorption of a photon (excitation laser); (ii) scattering of the free carrier, by two phonons with equal and opposite momentum $(q \neq 0)$, to (and from) an intermediate excited electronic state; and finally (iii) an electronic relaxation (with no change in momentum) accompanied with the emission of a photon with an energy difference equivalent to the sum of the energies of each phonon involved in the process. In order to achieve the double resonance (DR) condition, all the initial and final states of each electronic transition have to be real (instead of virtual) states. Even if the electronic states are virtual, but very close to a real state, a quasi-resonant condition could be achieved. Therefore, the DR condition will depend on the electronic band structure, phonon dispersion and laser excitation energy.

Resonant Raman scattering in bulk $\mathrm{MoS}_{2}$ and $\mathrm{WS}_{2}$, which are indirect band gap semiconductors, has been studied in the past by using excitation wavelengths close to the 
absorption edge. mainly red and near-IR excitation wavelengths [82, 185]. Under this condition, additionally to the first-order Raman modes, several other Raman peaks corresponding to multi-phonon modes appear in the spectrum. As we know, the electronic band structure for few-TLs of TMDs are different from the bulk [187] and evolve into a direct band gap semiconductor as the number of layers approaches unity. Consequently, the conditions required for resonant Raman scattering in a single-TL are different from the bulk.

Only recently, a pronounced double resonant Raman behavior was observed in single-TL $\mathrm{WS}_{2}$ [79]. Different from the bulk, in single-TL $\mathrm{WS}_{2}$ this condition was achieved with a $514.5 \mathrm{~nm}$ green excitation laser. When wavelengths of $488 \mathrm{~nm}$ and 647 $\mathrm{nm}$ were used, the most prominent Raman peaks corresponded to the first-order Raman modes $A 1 g(\Gamma)$ and $E 2 g 1(\Gamma)$. However, when using the intermediary wavelength 514.5 $\mathrm{nm}$, the intensity of the second-order Raman peak $2 L A(M)$ increases as the number of TLs decreases, being a maximum for a monolayer (Figure 4e-f) and stronger than that of the first-order modes. $L A(M)$ is a zone-edge longitudinal acoustic mode at the $\mathrm{M}$ point of the Brillouin zone. Since other semiconductor $\mathrm{MX}_{2}$ compounds suffer similar modifications in the band structure when transitioning to monolayers, it is possible to find the double resonant condition also for these 2D compounds when using, for instance, a laser with a tunable wavelength (e.g. dye lasers). This method is highly sensitive to changes in the electronic band structure and for this reason also serves as an alternative fingerprint for TMD monolayer identification. 


\section{Optical phenomena of monolayer and few-layer TMDs}

\subsection{Band to band photoluminescence}

Band to band photoluminescence (PL) in single layer TMDs is the physical phenomenon that demonstrates the existence of a direct band gap in many TMDs. The band to band PL was first observed in mechanically exfoliated single layer $\mathrm{MoS}_{2}$ in early 2010 by two independent groups. $[60,61]$ The main feature of the PL spectrum of monolayered $\mathrm{MoS}_{2}$ is an intense peak located at $675 \mathrm{~nm}(1.85 \mathrm{eV})$ with a FWHM of around $70 \mathrm{meV}$ (see Figure 5a). Ever since that remarkable observation, TMDs have attracted a great deal of interest because of their potential for multiple and varied applications in electronic and optical devices [89, 205-209].
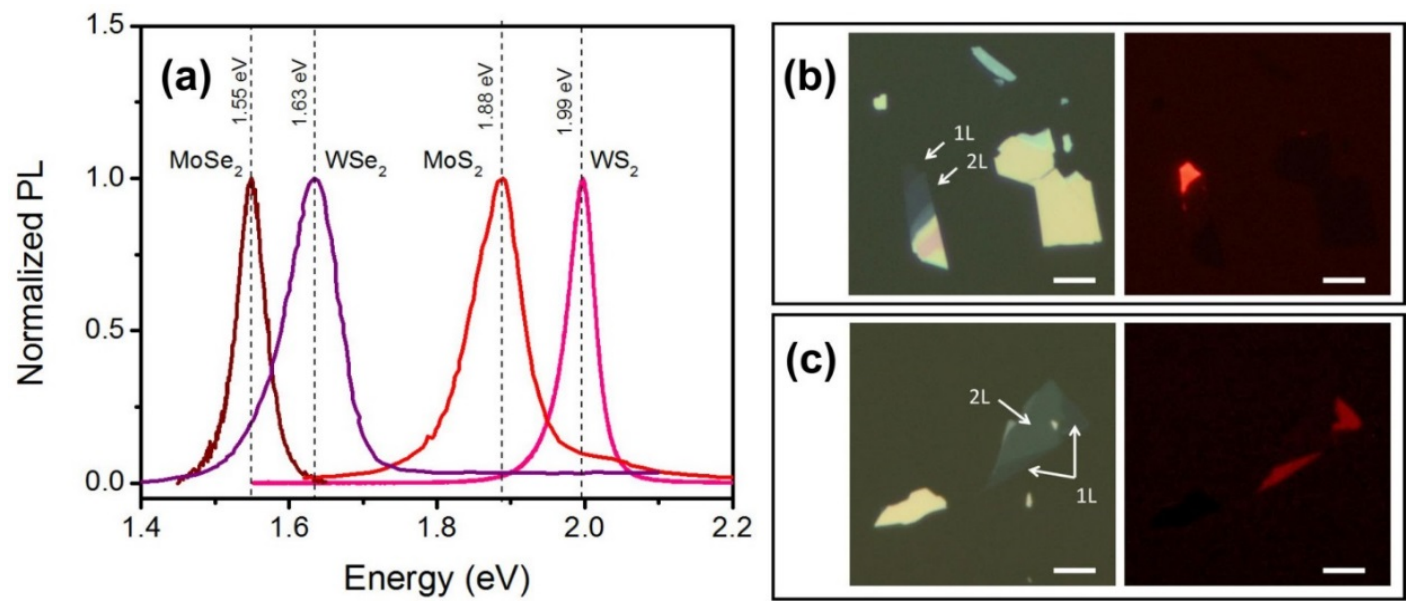

Figure 5 (a) Normalized PL spectra of Single layer TMDs. The data in this figure was extracted with permission from Ref [60] (Copyrights 2013 American Chemical Society) for $\mathrm{MoS}_{2}$, Ref [76] (Copyrights 2012 American Chemical Society) for $W_{2}$, Ref [91] (Copyrights 2013 American Chemical Society) for $\mathrm{WSe}_{2}$ and Ref [87] (Copyrights 2010 American Physical Society) for $\mathrm{MoSe}_{2}$. Bright field and fluorescence microscopy images of exfoliated $\mathrm{MoS}_{2}$ (b) and $\mathrm{WSe}_{2}$ (c). All the scale bars are $5 \mu \mathrm{m}$. Both (b) and (c) are reprinted with permission from Ref. [77]. Copyrights 2013 American Chemical Society. 
Simple optical techniques such as ellipsometry are very reliable and have been used for measuring the thickness of nanometer-thick films; however, when dealing with samples as thin as one or few atoms thick, like graphene and monolayers of TMDs, the ellipsometry technique is not useful. That is one of the reasons why PL has played such a very important role in the rapid development of the TMDs [59-61, 76, 87, 90, 137]. It is more convenient to perform a nondestructive spectroscopic measurement such as PL to determine the thickness of a flake or a film compared to other techniques, like AFM or TEM, which tend to compromise the intrinsic quality of samples. Furthermore, fluorescence microscopy which uses optical filters to select specific excitation ranges also filters the light coming out from the sample to the detector to selectively collect this light and to create an emitted light image. This technique has demonstrated to be flawless in detecting single layers TMDs like $\mathrm{MoS}_{2}, \mathrm{WS}_{2}$ and $\mathrm{WSe}_{2}$ in this connection. Figures 6.1b-c show the bright field and fluorescence images that are useful to locate the single layer portion of a given flake [77]. The first PL studies of isolated single layers of TMDs were performed on mechanically exfoliated samples because of the relative simplicity to obtain such samples[59]. However, when the CVD synthesis methods for single layer TMD determination were refined $[154,162]$, even more interesting results were obtained. Monocrystalline CVD grown samples of single layer TMDs, such as $\mathrm{WS}_{2}, \mathrm{MoS}_{2}$ and $\mathrm{WSe}_{2}$ exhibit perfectly shaped triangular islands $[76,162]$, and furthermore, it was found that under some specific growth conditions, multiple crystals merge in forming symmetric shapes. CVD grown samples also allowed study of the PL originating from well-defined crystalline edges and grain boundaries [162]. Particularly, for $\mathrm{WS}_{2}$ it was found that enhanced PL emission occurred from the edges of these monolayered triangles 
[76]. In these $\mathrm{WS}_{2}$ monocrystals, the PL intensity is centered at $635 \mathrm{~nm}(1.96 \mathrm{eV})$ and the PL intensity on the edges can be up to 25-40 times more intense than that observed in the central area and two orders of magnitude more intense than the very special Raman peaks with $\mathrm{A}_{1}{ }^{\prime}{ }_{\mathrm{g} g}$ and E' symmetry [76].

Table 2 lists the main reports regarding PL from single- and few-layer TMDs including the most important sample characteristics and PL emission properties. A general trend is observed in the spectral dispersion of the PL (FWHM), and it is evident that the PL from mechanically exfoliated samples have almost twice the FWHM linewidth, when compared with the corresponding FWHM coming from CVD grown monolayers. But there are many factors affecting the intensity, the distribution of PL emission energies and the energy of corresponding to PL of a monolayer TMD. For example, the PL emission is sensitive to temperature by an effect related to the depletion of thermally generated minority carriers and to the temperature dependence of the lattice parameters of TMDs. It has been demonstrated that the PL emission from $\mathrm{MoS}_{2}$ shifts by $90 \mathrm{meV}$ to higher energies (blueshift) when the sample temperature is dropped to $4.5 \mathrm{~K}$ [210]. Another mechanism that has been demonstrated to affect the PL emission is the surrounding atmosphere, of the TMD monolayer sample. For example, recently, Tongay S. et al. [91] performed an extensive study of the effect of $\mathrm{Ar}, \mathrm{N}_{2}, \mathrm{O}_{2}$ and $\mathrm{H}_{2} \mathrm{O}$-rich atmospheres on the PL emission of mechanically exfoliated single layer (SL) TMD samples that were thermally annealed in vacuum. In their study, these authors [91] found that specifically $\mathrm{O}_{2}$ and $\mathrm{H}_{2} \mathrm{O}$ molecules act as a "molecular gate" by modifying the charge density in the SL-TMD. They further found that a specific environment can produce an enhancement effect on the PL intensity for $\mathrm{MoS}_{2}$ could have an opposite effect on the 
$\mathrm{WSe}_{2}$ [91]. In a similar way they found that measurement of the changes in the PL from $\mathrm{MoS}_{2}$ exfoliated flakes caused by the effect of an insulating layer on top of either $\mathrm{Al}_{2} \mathrm{O}_{3}$ or $\mathrm{HfO}_{2}$ provided a simple way to test the integrity of the TMD after subsequent depositions for electronic device fabrication [211]. Another approach found to modulate the PL emission has been by creating alloys of molybdenum and tungsten disulfide. In this context, Chen $\mathrm{Y}$. et al. synthesized a series of $2 \mathrm{D}$ alloys of the system $\mathrm{Mo}_{1-\mathrm{X}} \mathrm{W}_{\mathrm{X}} \mathrm{S}_{2}$ and demonstrated a clear modulation of the direct band gap over the entire $0<\mathrm{X}<1$ composition range, as was evident from the shift of the PL peak from $1.99 \mathrm{eV}$ to $1.85 \mathrm{eV}$ over this composition range $[162,212]$. The Terrones group also demonstrated tunable PL signals depending on the stoichiometries of 2-D $\mathrm{Mo}_{1-\mathrm{X}} \mathrm{W}_{\mathrm{X}} \mathrm{S}_{2}$, which will open up a wide range of applications in electronics and optoelectronics [213]. In addition to synthetic, environmental and alloying effects on the PL emission, there is a strong effect of the light polarization on the PL of single layer TMDs, but given the importance of the phenomena and the great possibilities that alloying introduces, this topic will be discussed in the next section, which is related with valley polarization effects.

Table 2 List of different PL studies on monolayer TMDs and their photon emission characteristics.

\begin{tabular}{|c|c|c|c|c|c|c|c|}
\hline Material & $\begin{array}{l}\text { Synthesis } \\
\text { Method }\end{array}$ & $\begin{array}{l}\mathrm{T} \\
(\mathrm{K})\end{array}$ & $\begin{array}{l}\text { FWHM } \\
(\mathrm{meV})\end{array}$ & $\begin{array}{l}\text { Peak } \\
\text { Position }\end{array}$ & $\begin{array}{c}\text { No. } \\
\text { Layers }\end{array}$ & Substrate & Ref. \\
\hline $\mathrm{MoS}_{2}$ & $\begin{array}{l}\text { Mechanical } \\
\text { exfoliation }\end{array}$ & 300 & 51 & $1.88 \mathrm{eV}$ & 1 & $\begin{array}{l}\text { Suspe } \\
\text { nded }\end{array}$ & {$[60]$} \\
\hline $\mathbf{M o S}_{2}$ & $\begin{array}{l}\text { Mechanical } \\
\text { exfoliation }\end{array}$ & 300 & $>100$ & 1.83 & 1 & $\begin{array}{l}\mathrm{SiO}_{2} / \\
\mathrm{Si}, \\
\text { Quart } \\
\text { z }\end{array}$ & {$[61]$} \\
\hline $\mathbf{M o S}_{2}$ & $\begin{array}{l}\text { Mechanical } \\
\text { exfoliation }\end{array}$ & $\begin{array}{l}4.5 \text { to } \\
300\end{array}$ & $\sim 100$ & $\begin{array}{ll}1.88 & @ \\
4.7 \mathrm{~K} & \\
1.81 & @\end{array}$ & 1 & $\begin{array}{l}\mathrm{SiO}_{2} / \\
\mathrm{Si}\end{array}$ & [210] \\
\hline
\end{tabular}


$300 \mathrm{~K}$

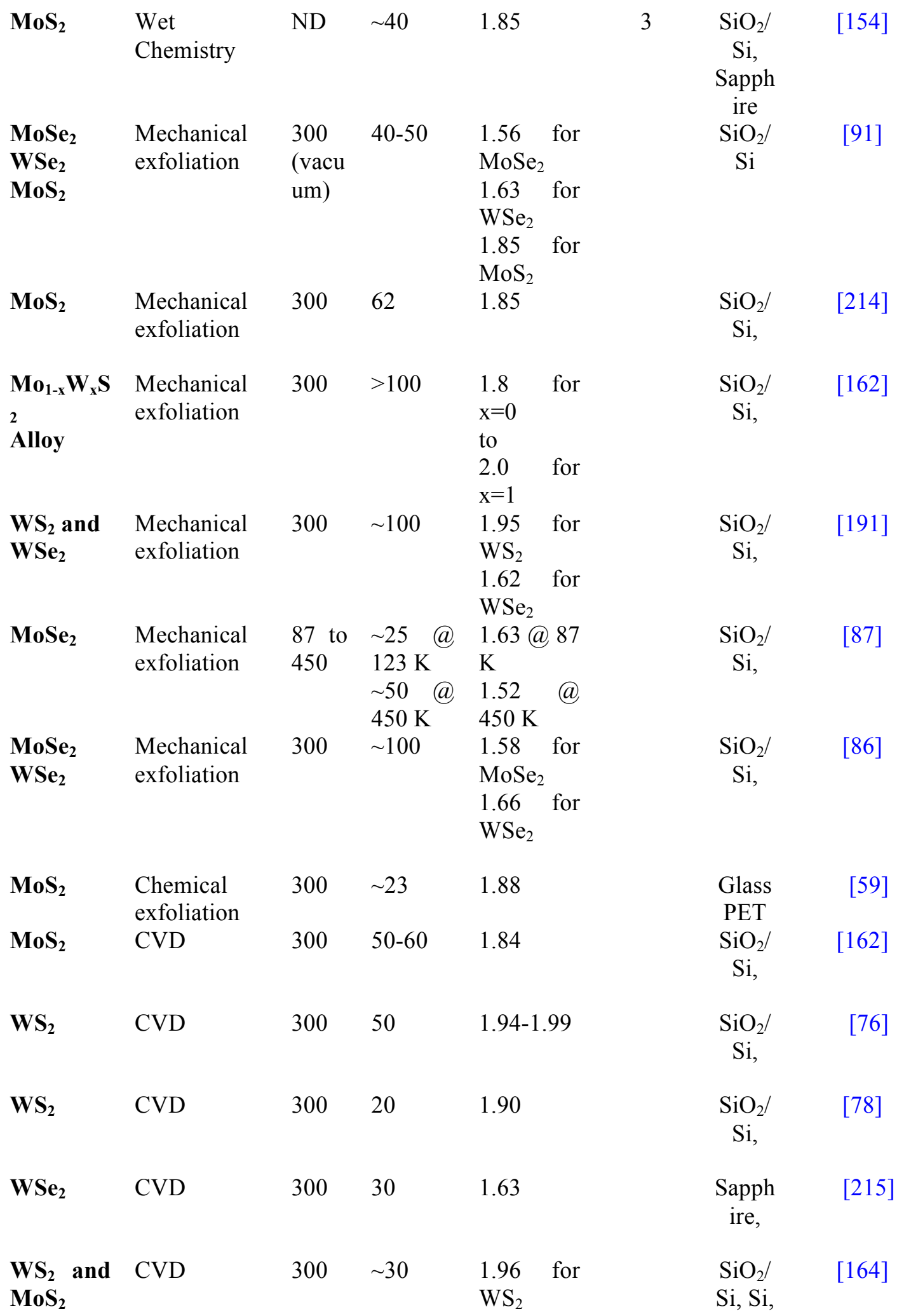




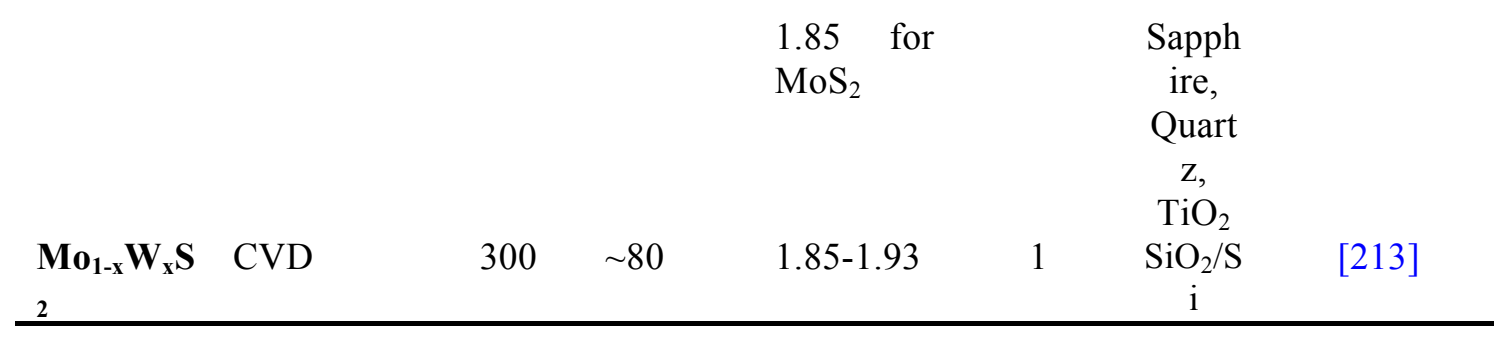

\subsection{Valleytronics}

Two dimensional materials offer the possibility to manipulate charge carriers (electrons and holes) using other properties in addition to the electric charge and spin. The study of such this phenomena is enabled by the lack of inversion symmetry present in the unit cell of monolayers of these materials. First, we note that the theoretical studies of the electronic band structure of graphene led to the discovery of the energy degenerate states at the valence band maximum (conduction band minimum) that enabled the realization of valley polarization effects [216-219]. By means of computational modeling, Rycerz et al. found that because of the very special electronic structure of very thin graphene stripes, it is possible to selectively populate a preferred valley by applying an electric field in a gated device consisting of a very narrow graphene stripe between two electrodes (quantum point of contact), thus enabling the possibility of the having an electron current with defined spin manipulated by valley polarization. [218, 219]

Despite the fact that graphene was the first material envisaged to be able to exhibit valley polarization, the experimental observation of this phenomena has not been achieved so far in that material, mainly because of the splitting of the bands is very small $(\mu \mathrm{eV})$ and the fact that graphene lacks of a band gap capable of producing luminescence in the visible or near IR limit the use of graphene for valleytronic application. 
Furthermore, the valley splitting is much smaller than the energy of thermally generated charge carriers at $300 \mathrm{~K}(\sim 26 \mathrm{meV})$.

With the advent of other layered materials analogous to graphene, specifically monolayers of TMDs, the theoretical study of the valley properties in such systems leads to the discovery of enhanced valley splitting produced by a giant spin-orbit coupling present in these materials $[117,118]$. Semiconducting layered materials of the type $\mathrm{MX}_{2}$ ( $\mathrm{M}=\mathrm{W}$ or $\mathrm{Mo}$ and $\mathrm{X}=\mathrm{S}$ or $\mathrm{Se}$ ) have the advantage of having strong band to band $\mathrm{PL}$ emission in the visible or near IR spectral regions, promoted by their direct band gap. Such emission is sensitive to circularly polarized light excitation, and enabled the first experimental observation of valley polarization in $\mathrm{MoS}_{2}$ monolayers [120, 121, 220]. Figure 5a shows the unit cell of $\mathrm{MoS}_{2}$ where the lack of inversion symmetry is evident.

According to density functional theory, the $\mathrm{K}$ and $\mathrm{K}$ ' points of the Brillouin zone host the extremes of the valence and conduction bands in $\mathrm{MoS}_{2}$ (and other $\mathrm{MX}_{2}$ semiconductors). The direct band gap transitions of light absorption and light emission by radiative recombination occur at these sites of momentum space. Additionally, the lack of inversion symmetry and the existence of time reversal symmetry in $\mathrm{MX}_{2}$ semiconductors promotes the coupling of spin and valley at the corners of the Brillouin zone ( $\mathrm{K}_{+}$and $\left.\mathrm{K}_{-}\right)$, as presented in Figure 6b, where the first Brillouin zone of $\mathrm{MoS}_{2}$ is shown in color to represent the different (+ and -) spins coupled to each nonequivalent point $\mathrm{K}_{+}$and $\mathrm{K}$. [120]. In such a situation, circular dichroism was first measured in single layer $\mathrm{MoS}_{2}$ by Cao T. et al. [120]. Circular dichroism (CD) is the optical phenomenon whereby circularly polarized light, with either right $\left(\sigma_{-}\right)$or left $\left(\sigma_{+}\right)$, circular polarization is absorbed at different rates by a material. Almost simultaneously, two other groups 
independently reported the observation of valley polarization promoted by optical excitation with circularly polarized light in monolayer $\operatorname{MoS}_{2}[121,123]$. Specifically, Zeng H. et al. demonstrated that the origin of the circularly polarized PL measured from $\mathrm{MoS}_{2}$ was not sensitive to a parallel magnetic field (see Figure 6c); in this way, the measurement eliminated the presence of the Hanle effect, as observed in other semiconductors where the PL polarization can be manipulated by an external magnetic field [123]. Another contribution in the study of the valley polarization effect was study of the temperature dependence of the valley polarization. In particular, Figure $6 \mathrm{~d}$ shows the behavior of the maximum polarization. Here we use that the maximum polarization has an almost constant value of $32 \%$ below $82 \mathrm{~K}$, and it decays rapidly above that temperature and approaches zero at room temperature. Lastly, Mak K. F. et al. [121] studied the behavior of the PL helicity ( $\rho$ ), which is the degree of PL polarization, as a function of the wavelength of the PL, finding that the value of the PL is a maximum $(\rho=1)$ when the excitation energy matches the A excitons of $\mathrm{MoS}_{2}$. Furthermore, these authors [121] also explored the effects of the substrate on the PL intensity when using h-BN and $\mathrm{SiO}_{2} / \mathrm{Si}$ substrates, and they found that the helicity is practically insensitive to the substrate as depicted in Figures 6.2e [121]. A Subsequent study explored a wider range of temperatures down to $4 \mathrm{~K}$ as well as higher magnetic fields up to $9 \mathrm{~T}$, but the results remained basically unaltered, thereby proving the robustness of the phenomena even at higher temperatures [221]. 


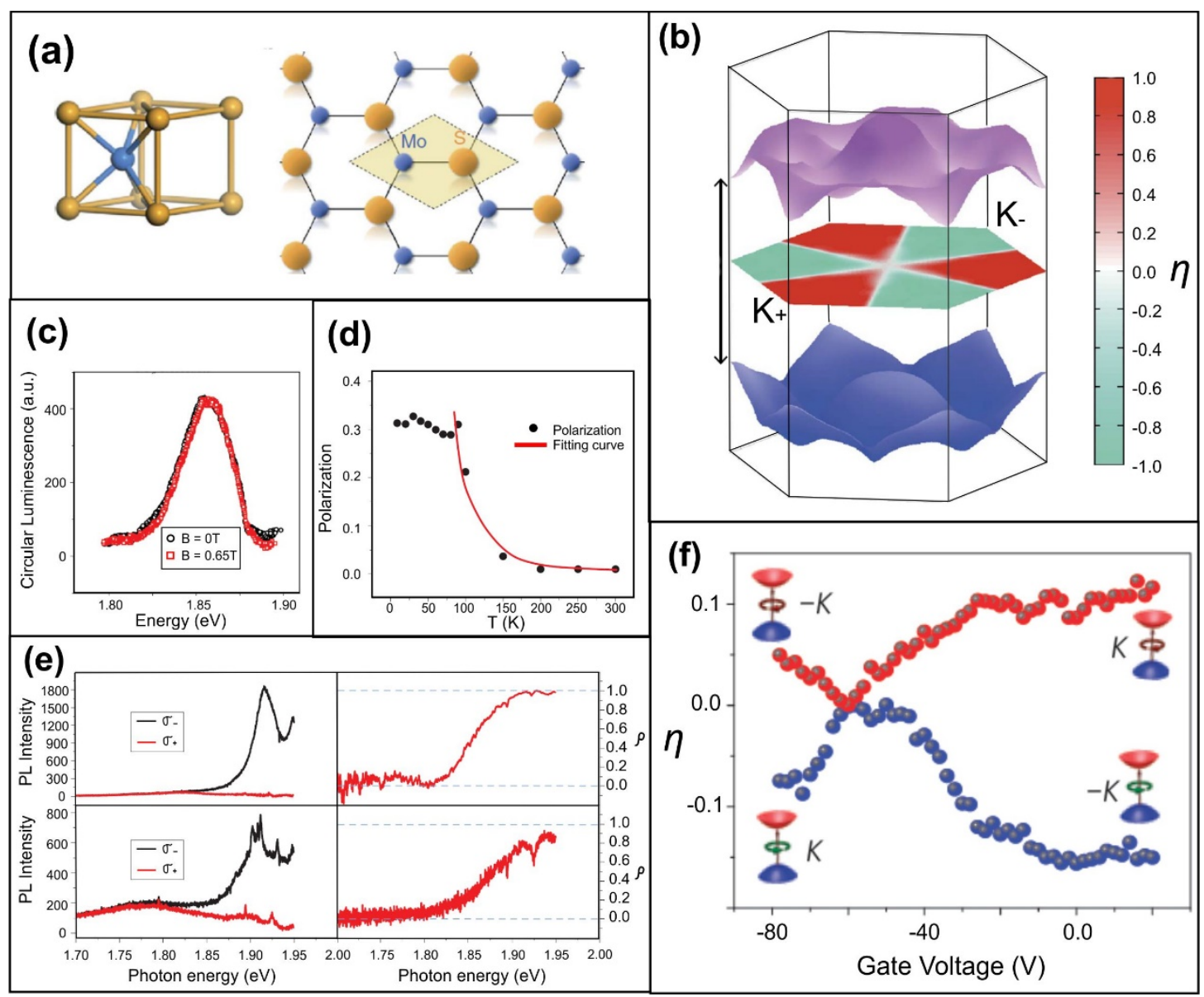

Figure 6 (a) Crystalline structure of $\mathrm{MoS}_{2}$, showing the Mo atom (blue) and its coordination with $S$ (yellow) atoms. On the right panel, the top view shows how two $\mathrm{S}$ atoms overlap but the hexagonal structure of $\mathrm{MoS}_{2}$ is more evident in this view. (b) The 3D (tridimesional) band structure of monolayer $\mathrm{MoS}_{2}$ is shown, the hexagon in the midlle between the valence (bottom) and conduction (top) bands is colored to show the degrre of polarization associated with each $\mathrm{K}$ and $\mathrm{K}^{\prime}$ point of the Brillouin zone, panels (a) and (b) are reprinted with permission from Ref. [120]. Copyrights 2012 Nature Publishing Group. (c) circularly polarized PL under different in plane magnetic field where the spectra match for 0 , and $0.65 \mathrm{~T}$, showing that the effect is independent of the magnetic field. (d) Temperature dependence of the degree of circular polarization showing a decay above $100 \mathrm{~K}$. Panels (c) and (d) are reprinted with permission from Ref. [123]. Copyrights 2012 Nature Publishing Group. (e) Comparision of the PL helicity for $\mathrm{MoS}_{2}$ on h-BN and $\mathrm{SiO}_{2} / \mathrm{Si}$ substrates showing no significative difference is found for different substrates. Reprinted with permission from Ref. [121]. Copyrights 2012 Nature Publishing Group. (f) Gate voltage controlled degree of PL polarization in $\mathrm{MoS}_{2}$ excited at $648 \mathrm{~nm}$. Reprinted with permission from Ref. [167]. Copyrights 2013 American Chemical Society. 
The observation of valley polarization was limited to mechanically exfoliated samples, with all samples coming from mineral sources. It is very likely that the magnitude of the polarization is dependent on the quality of the samples. The polarization reported from different groups $[120,121,123]$ were produced by the small degree of control that one has over the sample purity when working with mineral sources for samples. More recently the CVD synthesis methods have yielded samples with high quality that exhibit unit valley polarization at low temperatures $(80 \mathrm{~K})$ and even $40 \%$ of this polarization remains at room temperature [167].

The spin splitting that leads to valley polarization in single layers of $\mathrm{MoS}_{2}$ (and hypothetically in the other $\mathrm{MX}_{2}$ family of TMD materials) was well characterized by optical techniques $[120,121,123]$. However, a very important step is to transform this optical signal into electrical signals, and vice versa, in a way that we could use that additional degree of freedom of the charge carriers to convey valuable information. Recently, $\mathrm{Wu} \mathrm{S}$. et al. performed experiments where they electrically controlled the degree of valley polarization [122]. This was achieved by applying an external electric field using an FET structure constructed with bilayer $\mathrm{MoS}_{2}$ as the channel. The basic idea of this work [122] is related to breaking the symmetry of bilayer $\mathrm{MoS}_{2}$; in equilibrium, bilayer $\mathrm{MX}_{2}$ has inversion symmetry, which suppresses the spin valley coupling, but in the presence of an external electric field, the inversion symmetry can be broken and valley polarization can appear. In their results [122], they varied the degree of polarization from 0 to $15 \%$ by applying a gate voltage from $-60 \mathrm{~V}$ to $20 \mathrm{~V}$ while the sample was exposed to left circular polarized light $\left(\sigma^{+}\right)$excitation. A similar result was 
observed with right $\left(\sigma^{-}\right)$polarized light but for opposite signs, as can be seen in Figure $6 f$ [122].

Along the same direction, novel results on single layer $\mathrm{WSe}_{2}$ have demonstrated that by simultaneously applying an electric field and polarized optical pumping, it is possible to generate valley polarization and coherence. This work demonstrates experimentally that by applying an electric field in $\mathrm{WSe}_{2}$, it is possible to create charged excitons (trions). Those excitons are susceptible to being selectively excited by a predetermined polarized light (circular or linear) and their polarized PL response can be tuned with varying the gate electric field [222]. Similar to a field effect gate the surrounding dielectric environment can screen excitons and trions (charged excitons) which could allow the development of high efficiency valleytronics [223]. Finally, numerical calculations yield interesting predictions to build quantum bit devices based on valley control in semiconducting SL-TMDs [224]. However, it is clear that other semiconducting monolayers of TMDs as well as hybrid monolayers of TMDs now need to be studied under circularly polarized light.

\subsection{Non-linear optical properties}

\subsubsection{Second harmonic generation}

Another manifestation of the isolation of TMD monolayers is the change of their symmetry properties; bulk $\mathrm{MX}_{2}$ semiconductors exhibiting $2 \mathrm{H}$ stacking belong to the point group $D_{6 h}$, but for a monolayer it loses the inversion point symmetry operation, so it belongs to the point group $D_{3 h}$. The lack of inversion point symmetry is a prerequisite for efficient second harmonic generation (SHG). In the past, this non-linear optical property 
was explored in bulk TMDs finding no significant SHG for $10 \mu \mathrm{m}$ thick $2 H-\mathrm{MoS}_{2}$ [225], More recently, the study of optical SHG on exfoliated and restacked $\mathrm{MoS}_{2}$ crystallites intercalated with different amounts of malachite green (including pure $\mathrm{MoS}_{2}$ ) was used to correlate the interlayer distance as a function of the intercalant amount [226]. Here the optical SHG was presented as a complementary technique to XRD for the determination of the interlayer spacing in chemically exfoliated and restacked layered materials [226].

Zhang et al. observed SHG on mechanically exfoliated $\mathrm{WSe}_{2}$ and $\mathrm{WS}_{2}$ [191]. These authors provided experimental proof of the relation between the number of layers, inversion symmetry and the SHG. They found that when an odd number of layers were present, the efficiency of SHG was high and when an even number of layers were measured, the $\mathrm{SH}$ emission was below the detection limit. Therefore, SHG provides a useful technique to determine the sample thickness, and this technique is also able to distinguish between monolayer and few-layered TMDs; SHG is efficiently generated in mono-, triple- and penta-layers, while band to band PL is only efficient in monolayer TMDs (See Figure 7a-d). Subsequently, Malard et al. observed SHG on mechanically exfoliated $\mathrm{MoS}_{2}$ flakes [227], with the behavior of the SHG depending on the parity and the number of $\mathrm{MoS}_{2}$ layers in a similar way to that presented here for $\mathrm{WS}_{2}$ and $\mathrm{WSe}_{2}$. However, a brilliant approach was proposed by them when using linearly polarized light, whereby they were able to distinguish the crystalline orientation of the sample as schematized in Figure 7e. Such a structural distinction is possible because when the polarization vector is parallel to the zigzag direction, the SHG has a maximum; however, the opposite happens for the armchair direction which shows a minimum SHG for this case, as can be visualized in the polar plot of Figure $7 \mathrm{f}$ that shows the intensity of the 
SHG in monolayered $\mathrm{MoS}_{2}$. When non-polarized detection is used, the SH response is not sensitive to the sample orientation. Almost simultaneously Kumar et al. [228] realized in a similar study on both mechanically and CVD produced samples, and proposed a proof-of-principle for a second harmonic microscopy technique [228], a method capable of detecting the number of layers and its crystalline orientation in the sample as depicted in Figure 7e.

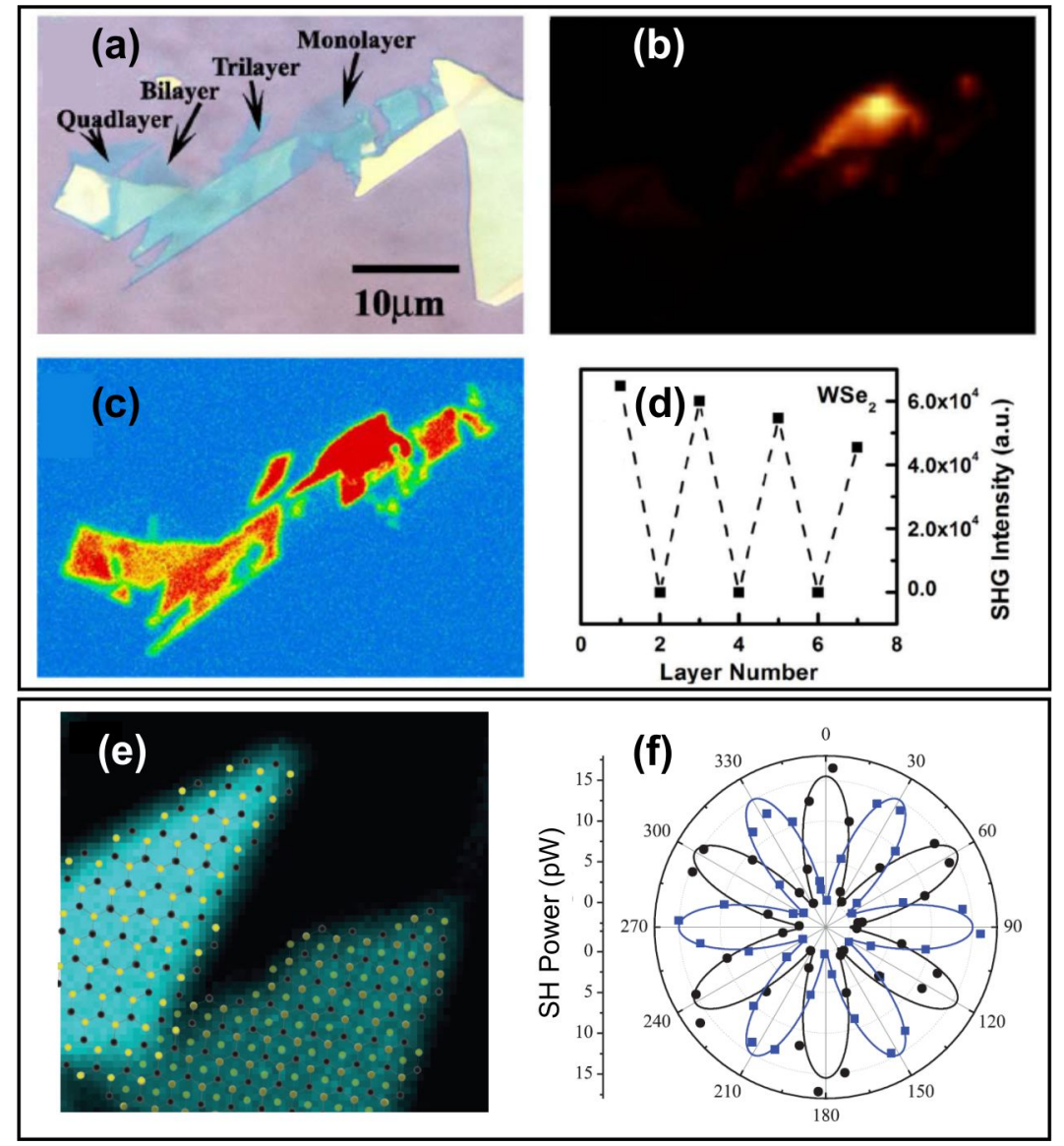

Figure 7 (a) Optical micrograph of mechanically exfoliated WSe $\mathrm{W}_{2}$. (b) PL image of the same area showing intense red emission in the single layer region. (c) Second harmonic generation (SHG) image map of the same area as in (a), notice that all regions with an odd number of layers show up and the even number of layers are suppressed. (d) Oscillation of the SHG intensity as a function of the number of layers. Panels a-d are reprinted with permission from Ref. [191]. Copyrights 2013 Nature Publishing Group. (e) SHG image of a single layer and tri-layer regions of $\mathrm{MoS}_{2}$. The overlay is the associated crystalline orientation for each region obtained from polarization resolved SHG. Reprinted with permission 
from Ref. [227]. Copyrights 2013 American Physical Society. (f) Polar plot of SH power for the parallel (black dots) and perpendicular (blue squares) components of the $\mathrm{SH}$ emission. The continuous lines are the expected dependence for CVD $\mathrm{MoS}_{2}$. Reprinted with permission from Ref. [228]. Copyrights 2013 American Physical Society.

A good demonstration of the usefulness of identifying the crystallographic orientation was provided by He et al. [229]. Using polarization effects, they were able to locate and orient a microscopic foil of $\mathrm{MoS}_{2}$ on a macroscopic polymethylmethacrylate (PMMA) sheet in order to study the effects of uniaxial strain on different crystallographic orientations of single layer $\mathrm{MoS}_{2}$. Polarization resolved SHG is a novel technique that will be valuable for the future device integration of single and few layered TMDs on a large production scale [230-232].

Table 3 Survey of the relevant studies of second harmonic generation from TMDs, including some of the key conditions used for each measurement.

\begin{tabular}{|c|c|c|c|c|c|c|c|}
\hline Material & Substrate & $\begin{array}{l}\text { Pump } \\
\lambda \\
(\mathrm{nm}) \\
\end{array}$ & $\begin{array}{l}X^{(2)} \\
\mathrm{pm} / \mathrm{V}\end{array}$ & $\mathbf{T}$ & $\begin{array}{l}\text { Synthesis } \\
\text { method }\end{array}$ & Polarization & Reference \\
\hline $\begin{array}{l}2 \mathrm{H}-\mathrm{MoS}_{2} \\
\text { in bulk }\end{array}$ & NA & 1064 & $5 \times 10^{-2}$ & RT & $\begin{array}{l}\text { Natural (10 } \\
\mu \mathrm{m} \text { thick) }\end{array}$ & $\begin{array}{l}\text { Non } \\
\text { polarized }\end{array}$ & $\begin{array}{l}\text { Wagoner } \\
{[225]}\end{array}$ \\
\hline $\begin{array}{l}\mathrm{MoS}_{2} / \\
\text { MG }\end{array}$ & Quartz & 800 & NA & RT & $\begin{array}{l}\mathrm{Li} \\
\text { intercalation }\end{array}$ & Linear & $\begin{array}{l}\text { Cetnarowski } \\
\text { [226] }\end{array}$ \\
\hline $\begin{array}{ll}\mathbf{W S}_{2} & \& \\
\mathbf{W S e}_{2} & \end{array}$ & $\begin{array}{l}\mathrm{SiO}_{2} / \mathrm{Si} \\
(300 \mathrm{~nm}) \& \\
\text { Quartz }\end{array}$ & 800 & NA & RT & Mech Exf & $\begin{array}{l}\text { Non } \\
\text { polarized }\end{array}$ & Zeng [191] \\
\hline $\mathrm{MoS}_{2}$ & $\begin{array}{l}\mathrm{SiO}_{2} / \mathrm{Si} \\
(300 \mathrm{~nm}) \& \\
\text { Quartz }\end{array}$ & $\begin{array}{l}800, \\
860 \\
900 \\
960\end{array}$ & $\begin{array}{l}8 \times 10^{4} \text { for } \\
1 \mathrm{~L} \\
6 \times 10^{4} \text { for } \\
3 \mathrm{~L}\end{array}$ & RT & Mech exf & Linear & $\begin{array}{l}\text { Malard } \\
{[227]}\end{array}$ \\
\hline $\mathrm{MoS}_{2}$ & $\begin{array}{l}\mathrm{SiO}_{2} / \mathrm{Si} \\
90 \mathrm{~nm}\end{array}$ & 810 & $\sim 1 \times 10^{5}$ & RT & $\begin{array}{l}\text { Mech exf \& } \\
\text { CVD }\end{array}$ & Linear & $\begin{array}{l}\text { Kumar } \\
{[228]}\end{array}$ \\
\hline $\mathbf{M o S}_{2}$ & Pmma & 800 & NA & RT & Mech exf & Linear & $\mathrm{He}$ [229] \\
\hline $\begin{array}{l}\mathrm{MoS}_{2} \\
\mathrm{BN}\end{array}$ & Fused silica & 810 & NA & RT & Mech exf & Linear & $\operatorname{Li}[137]$ \\
\hline
\end{tabular}




\subsubsection{Broadband and ultrafast saturable absorption}

Saturable absorption (SA) is another type of non-linear optical phenomena induced by the imaginary part of the complex $3^{\text {rd }}$-order susceptibility, which is an important light-matter interaction and commonly observed in semiconductor materials [233]. When a semiconductor is excited by photons with energy larger than its bandgap, electrons will be transferred from the valance band to the conduction band. If the excitation energy is high enough, the final states will be fully occupied and show SA phenomena [233]. Traditional SA materials, such as Cr:YAG, V:YAG and GaAs can only respond to a given wavelength band. In order to develop high-performance photonic devices, it would be crucial to explore materials with broadband and ultrafast SA responses. Wang et al. demonstrated that few-layer $\mathrm{MoS}_{2}$ nanosheets can exhibit strong SA for the femtosecond (fs) pulses at $800 \mathrm{~nm}$ [234]. Furthermore, by introducing suitable defects, a broadband SA can be achieved for $\mathrm{MoS}_{2}$ [233]. Very recently, it was demonstrated that the SA properties of $\mathrm{MoS}_{2}$ and $\mathrm{WS}_{2}$ is size-dependent. Zhou et al. found that $\mathrm{MoS}_{2}$ and $\mathrm{WS}_{2}$ samples with flake sizes over $100 \mathrm{~nm}$ exhibit SA, while those with flakes smaller than 50 nm show strong reverse saturable absorption (RSA) [235]. More theoretical and experimental work needs to be carried out along this line in order to clarify the mechanism and develop possible applications of TMDs in photonics and optoelectronics.

\section{Other characterization techniques for TMDs}

Early reports on TMDs describe the use of conventional electron diffraction and convergent beam electron diffraction for studying the stacking order and crystal symmetry of the charge density wave states of these materials $[236,237]$. While the 
scalability of growing monolayer, bi-layer or multilayer of TMDs using a bottom-up or top-down approach still remains a challenge, a wide array of state-of-the-art characterization techniques are now available to analyze these materials. Most of the characterization tools that have also been used in graphene research can be used to characterize TMDs, including optical microscopy, Raman spectroscopy, high-resolution transmission electron microscopy (HRTEM), scanning transmission electron microscopy (STEM), atomic force microscopy (AFM), and scanning tunneling microscopy (STM) and X-ray photoelectron spectroscopy.

\subsection{HRTEM}

The use of transmission electron microscopy (TEM) as a tool for understanding two-dimensional layered materials dates back to the early 1900s, and since then, there has been a lot of progress in the HRTEM field owing to major advances in TEM instrumentation [68]. Fortunately, it is now possible to see materials with atomic resolution [238] as opposed to how, a few decades ago, image contrast had to be used to measure crystal thicknesses of TMDs [239]. Currently, HRTEM is one of the most popularly used characterization techniques in studying two-dimensional layered materials. It has a lot to offer in terms of understanding TMDs, including the determination of the number of layers, crystal structure, elemental composition and defects [76, 240].

In our previous work we have demonstrated the use of electron diffraction patterns (EDP) and phase contrast HRTEM to differentiate between regions of single crystal domains and those multilayer domains in triangular $\mathrm{WS}_{2}$ (Fig.8a-d) [76]. There have also been efforts to study the vacancy diffusion mechanism in $\mathrm{MoS}_{2}$ using TEM [240]. Defect 
agglomeration has been observed when increasing the vacancy concentration in $\mathrm{MoS}_{2}$ under electron irradiation in a HRTEM using a primary beam energy of $80 \mathrm{keV}$ (Figure 8e). Recent studies carried out in the field of van der Waals heterostructures based on graphene, report cross-sectional imaging in place of top-view imaging. A high resolution aberration corrected bright field STEM has enabled easier interpretation of atomic layer structural details of these materials (Fig 8f) [241]. Cross-sectional TEM imaging of TMDs has also been found to be useful for comparison with the film thickness data obtained from AFM measurements [76]. Similar studies can give valuable information when theoretically predicted heterostructures of layered TMDs with tunable band gaps become a reality [109].

\subsection{STM}

In 1982, Binnig et al. discovered this fascinating tool that now allows us to perform surface studies of materials with high atomic precision [242]. The working principle of an STM is based on the tunneling effect of current from the STM tip to the sample surface on applying a bias voltage, allowing us to study the surface details of the material. In the late 1980s, STM became very popular for resolving geometric and electronic structures of metallic and semiconducting materials [243]. Now, this is a widely used technique in the field of layered materials including graphene, h-BN and TMDs. For instance, images of atom-resolved $\mathrm{MoS}_{2}$ nano clusters on gold substrates obtained using STM have been found to exhibit a triangular morphology (Fig.8g-h) [244]. The details of the edge termination patterns in these triangular clusters have also been investigated. STM has also been used for studying the crystallinity of graphene films by studying the occurrence 
of superstructures due to lattice distortions [245]. In addition, an STM tip has the potential for use as a nanofabrication device. There are many reports on how such tips are used to generate atomic scale nanostructures on TMDs by the application of voltage pulses between the STM tip and the sample [246].
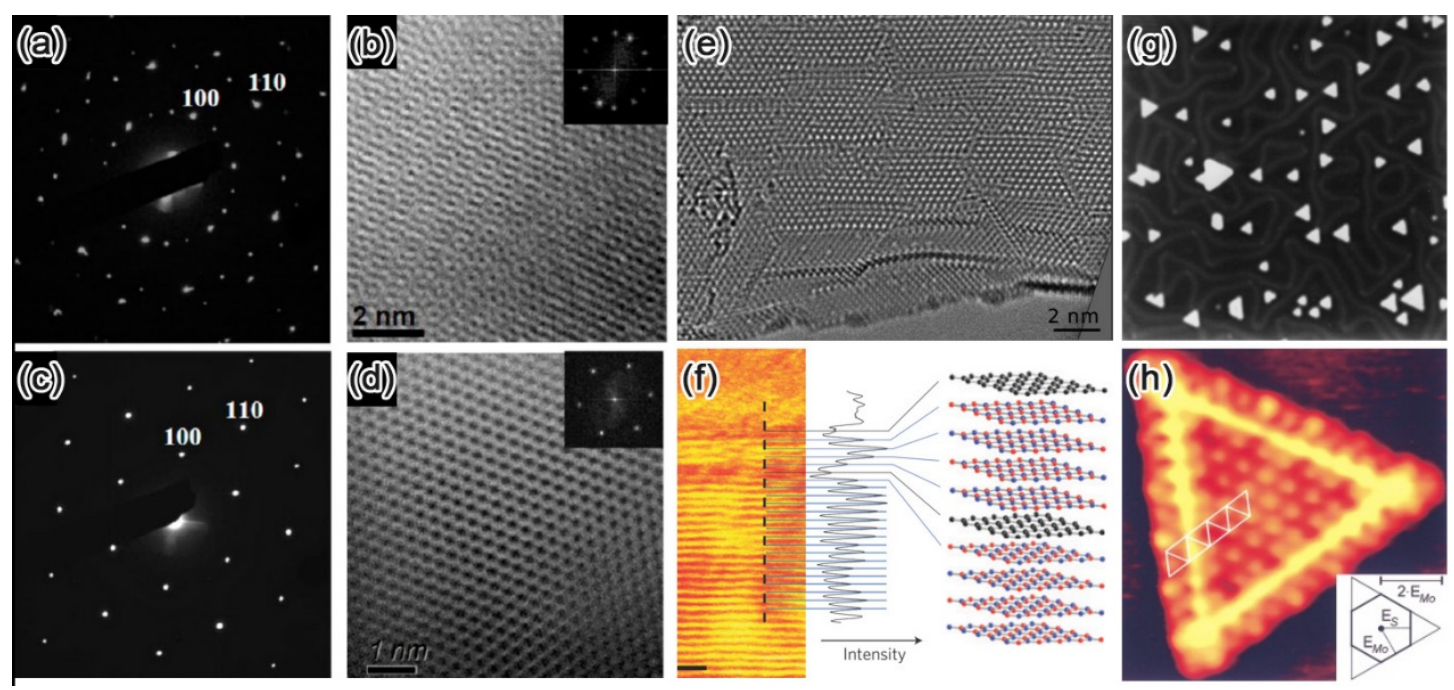

Figure 8 (a-b) Electron diffraction and HRTEM images, respectively, of a folded region of $\mathrm{WS}_{2}$ islands in comparison with single crystal domains of the same material shown in (c) and (d). Reprinted with permission from Ref. [76]. Copyrights 2013 American Chemical Society. (e) High concentration of line defects observed in HRTEM studies of freestanding $\mathrm{MoS}_{2}$ monolayers on irradiation with a $80 \mathrm{keV}$ electron beam for $220 \mathrm{~s}$. Reprinted with permission from Ref. [240]. Copyrights 2013 American Physical Society. (f) Cross-sectional image illustrating an atomic layer sequence of a Graphene-h-BN heterostructure obtained using a high-resolution bright field aberration corrected STEM. The dashed black line corresponds to the region where an averaged line scan of the intensity was taken and a schematic of the layer sequence is given on the right side of (f). Color coding is as follows: black, blue, and red for carbon, boron, and nitrogen atoms, respectively. Reprinted with permission from Ref. [241]. Copyrights 2012 Nature Publishing Group. In (g) and (h), STM images of triangular shaped MoS $_{2}$ clusters: (g) $744 \AA \times 721 \AA$ and (h) $41 \AA \times 42 \AA$. A Wulff construction of a $\mathrm{MoS}_{2}$ crystal is shown in the inset of (h). Reprinted with permission from Ref. [244]. Copyrights 2000 American Physical Society. 


\subsection{AFM}

AFM is also very useful when determining layer thickness, area and when generating a three dimensional surface profile of a two-dimensional layered material on an insulating substrate. Usually, color contrast in optical images of layered TMDs can give a rough idea about the number of layers present in a TMD sample. However, using the AFM, a thorough investigation of the sample can be carried out using height profiling analysis (Fig 9a-i) [247]. In many cases, a combination of optical imaging, Raman spectroscopy and AFM studies has been used to confirm the growth or exfoliation of monolayers of TMDs [247, 248].

AFM has also been used to study the tribological properties of TMDs. For example, there have been investigations on the effects of surface treatments on the layer morphology of materials, such as $\mathrm{MoS}_{2}$ and $\mathrm{NbSe}_{2}$, using AFM [249].

\subsection{XPS}

X-ray Photoelectron Spectroscopy (or XPS, earlier known as Electron Spectroscopy for Chemical Analysis (ESCA)) can be used to gain information about the atomic content and different chemical environments of various elements present on the surface of a material. Being a surface sensitive technique, XPS provides a unique tool for studying two dimensional materials. Almost a decade ago, there was a lot of interest among TMD researchers to study the stoichiometry of these materials because it was known that their properties varied with composition. The chemical shift or binding energy variations gave valuable information in this regard since such effects are directly related to core level electrons and these properties are affected by any variation in the electrostatic screening 
of these core level electrons by valence electrons $[250,251]$. XPS data can also provide information about the extent of contamination in the sample from the substrate and environment $[252,253]$. For example, the XPS spectrum reported for a cleaved surface of $\mathrm{MoSe}_{2}$ single crystals is shown in Fig.9j. The binding energy values of Mo and Se are in good agreement with theoretical predictions, and the absence of contaminants such as $\mathrm{Si}$, $\mathrm{Cl}$ and Te were confirmed in this work [252].

\subsection{UV-Vis}

One of the interesting areas that scientists are trying to investigate in TMDs and other layered materials, is their optoelectronic properties. Optically induced electronic transitions are believed to change the optical energy gap in these materials. UV-Vis spectroscopy renders a straightforward technique in studying this phenomenon. The absorption spectrum obtained from UV-Vis spectroscopy can be used to determine the optical energy bandgap, whereas the relationship between the absorption coefficient and the photon energy provides information about the electronic transitions and band structure of materials [254]. This has been demonstrated for graphene and its derivatives in many reports [174]. Figure 9k shows UV-Vis absorption spectra of different concentrations of h-BNC films [238].

The optical energy band gap $\mathrm{E}_{\mathrm{g}}$ is calculated using Tauc's equation $[174,255]$ :

$$
\omega^{2} \varepsilon=\left(\hbar \omega-E_{g}\right)^{2}
$$

Here $\varepsilon$ is the optical absorbance, and $\omega$ is the angular frequency of the incident radiation. For example, the Tauc's formulation has been applied recently to calculate the 
energy gap of atomic layers of h-BN which yielded a value of $5.56 \mathrm{eV}$ which compared fairly well with a theoretical value of $6 \mathrm{eV}$ for a single layer h-BN (Figure 91) [174] .
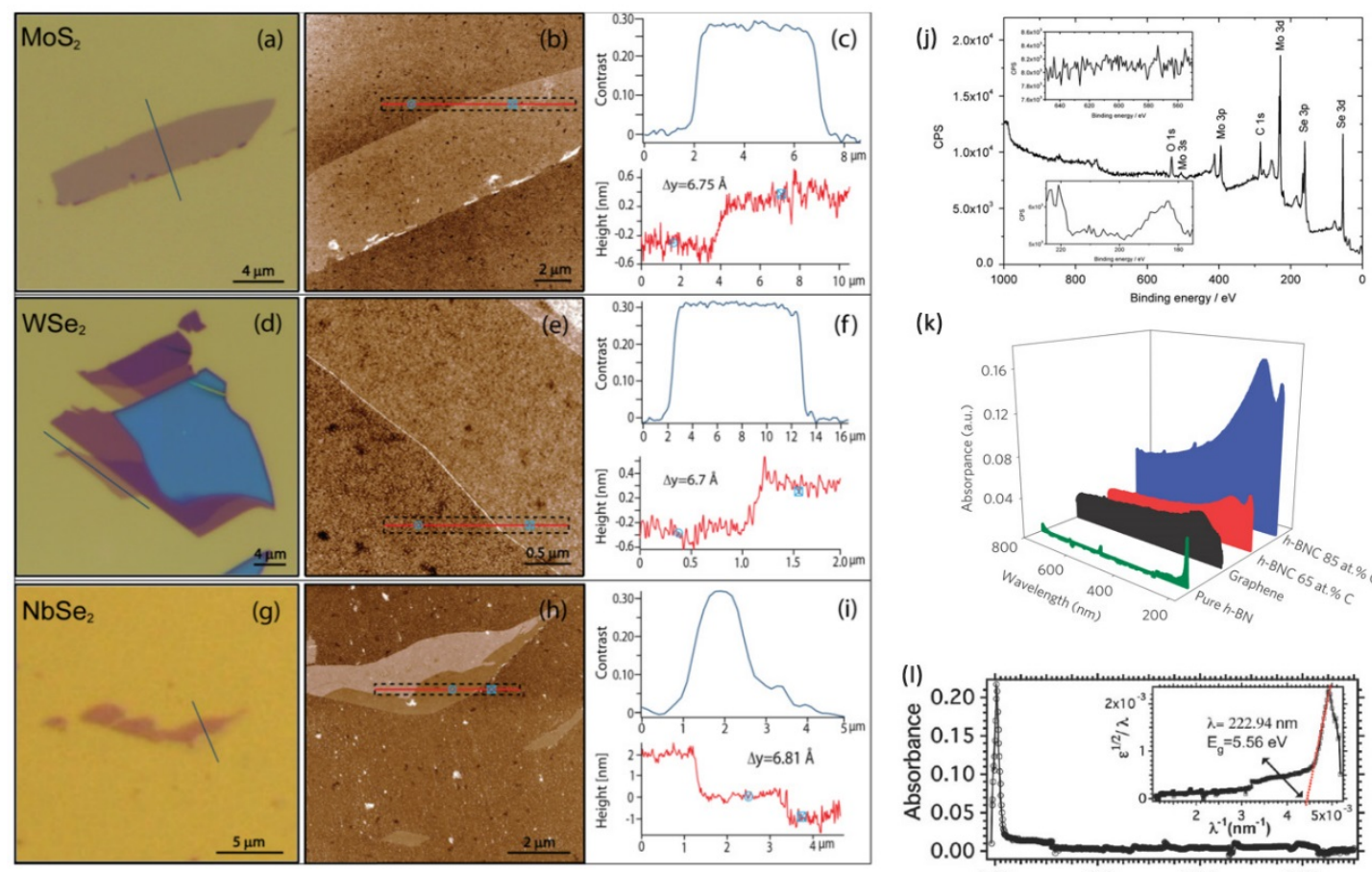

(k)
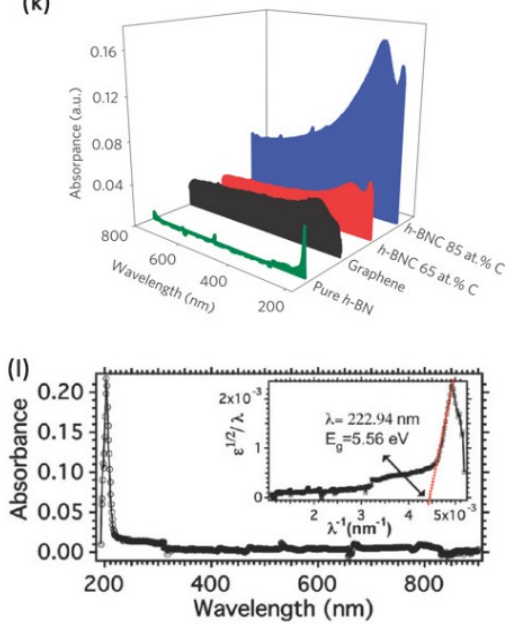

Figure 9 (a-i) Optical images and AFM line scan height profiles of $\mathrm{MoS}_{2}, \mathrm{WSe}_{2}$ and $\mathrm{NbSe}_{2}$ nanolayers on $270 \mathrm{~nm} \mathrm{SiO}{ }_{2}$. Layer thickness values obtained were $7.75 \AA$ for $\mathrm{MoS}_{2}, 7.7 \AA$ for $\mathrm{WSe}_{2}$ and $7.81 \AA$ for $\mathrm{NbSe}_{2}$ which correspond to single layer values. Reprinted with permission from Ref. [247]. Copyrights 2011 IOP Publishing Ltd. (j) XPS spectrum of a cleaved single crystal of $\mathrm{MoSe}_{2}$ showing characteristic $\mathrm{Mo}$ and Se peaks. The insets are characteristic peaks for $\mathrm{Te}$ and $\mathrm{Cl}$ showing the absence of these contaminants in the sample. Reprinted with permission from Ref. [252]. Copyrights 2013 Elsevier B.V. (k) UV-Vis absorption spectra of graphene, $\mathrm{h}-\mathrm{BN}$ and two different concentrations of h-BNC films showing a weak absorption edge for graphene at 190-800 nm, and h-BN at 218 $\mathrm{nm}$. Two absorption edges are seen for h-BNC 65 at.\%C and h-BNC 85 at.\%C, suggesting the presence of hybrid atomic layers of $h-B N$ and $C$ graphene nanodomains. Reprinted with permission from Ref. [238]. Copyrights 2010 Nature Publishing Group. (I) UV-Vis absorption spectra of h-BN showing a sharp absorption edge at $203 \mathrm{~nm}$. Inset shows a plot of $\varepsilon^{1 / 2} / \lambda$ versus $1 / \lambda$ and a calculation of the optical band gap using the Tauc's formulation. Reprinted with permission from Ref. [174]. Copyrights 2010 American Chemical Society. 


\subsection{Optical microscopy}

In addition to above-mentioned AFM, STM, HRTEM techniques, optical microscopy can also be used to identify the layer numbers of TMDs in a simple and fast way. Li et al. proposed to determine the layer numbers of nanosheets by comparing the optical contrast differences between the TMD nanosheets and the substrates (e.g. $\mathrm{SiO}_{2} / \mathrm{Si}$ ) [256]. Before starting identification, a standard reference needs to be established by collecting and tabulating the optical contrast differences and layer numbers. The former can be obtained by processing the color optical images of TMD nanosheets and the grayscale images of R, $\mathrm{G}$ and $\mathrm{B}$ channels with certain softwares (e.g. Image J). The latter can be obtained by AFM measurements on TMD nanosheets with different layer numbers. Once the reference is established, the layer numbers can be identified in a simple and rapid way, without needing any complex calculation or special instruments. This method has been applied to identify the layer numbers of mechanically exfoliated $\mathrm{MoS}_{2}, \mathrm{WSe}_{2}, \mathrm{TaS}_{2}$ and graphene nanosheets from monolayer to quindecuple-layer (1L-15L) on $\mathrm{Si}$ substrates coated with 90 or $300 \mathrm{~nm} \mathrm{SiO}_{2}$ [256]. This provides an efficient and cost-effective optical identification method for thickness-dependent research and applications on TMD nanomaterials.

\section{Optical and electronic devices based on TMDs}

The semiconducting TMDs $\left(\mathrm{MX}_{2} ; \mathrm{M}=\mathrm{W}\right.$ or $\mathrm{Mo}$ and $\mathrm{X}=\mathrm{S}$ or Se) are among the most promising materials for realizing the next generation electronic and optoelectronic devices. The first exploration of the electronic behavior of a monolayer of a TMD was reported in 2005 by the group of A. Geim and K. Novoselov [124]. In that seminal study, 
the authors explored the conductivity changes of single layers of $\mathrm{MoS}_{2}$ and other layered materials, and found that the carrier density could be modulated by applying a perpendicular electric field in a bottom gated field effect transistor device. Figure 10a shows the optical image of the device structure used in their study and the field induced conductivity change in the $\mathrm{MoS}_{2}$ film. The authors elucidated that 2D electronic materials could reach the development level then available for SWNTs.

A few years later, in 2010, it was discovered that the electronic structure of $\mathrm{MoS}_{2}$ crosses over from an indirect to a direct band gap when thinned to a single layer $[60,61]$. The same behavior was shortly after reported for other semiconducting TMDs such as $\mathrm{WS}_{2}$ and $\mathrm{WSe}_{2}[76,215]$. The discovery of a direct band gap attracted lots of attention towards the semiconducting monolayered TMDs because such materials give rise to unique optical phenomena including band to band photoluminescence (PL). As discussed above when using this property, a TMD monolayer could be unambiguously identified by a simple spectroscopic measurement. This discovery solved the very time consuming task of identifying monolayers using other techniques. In a very short period of time after this discovery TMDs were used to fabricate electronic devices that now include field effect transistors [247], diodes [257], phototransistors [258-261], solar cells [262], small signal amplifiers [263], logic gates [264] and photodetectors [139]. In the following section, the evolution of the use of semiconducting TMDs is presented.

\subsection{Field effect transistors (FET)}

The most basic electronic device architecture where a TMDs single layer can be used is in a field effect transistor (FET). These devices can be built simply by placing a TMD 
flake on a thin insulator such as that found on $\mathrm{SiO}_{2} / \mathrm{Si}$ wafers, and evaporating metallic electrodes for the source $(\mathrm{S})$ and drain $(\mathrm{D})$; the gate $(\mathrm{G})$ terminal is connected to the heavily doped silicon bulk wafer, as schematized in Figure 10b [89, 124, 138]. The use of $\mathrm{SiO}_{2}$ on $\mathrm{Si}$ offers the advantage of providing a good color contrast which facilitates the localization of the ultra-thin flakes for optical lithographic or e-beam lithography processing [124].

Initially, mechanically exfoliated monolayers of $\mathrm{MoS}_{2}$ were isolated by Radisavljevic and collaborators and were used as the channel in a FET [89]. In their design, a monolayer of $\mathrm{MoS}_{2}$ was contacted with gold leads deposited by means of electron beam lithography. The device had a back gate formed by the body of the silicon substrate and coupled through a $270 \mathrm{~nm} \mathrm{SiO} 2$ dielectric layer. Additionally, a top gate made of gold was coupled through a $30 \mathrm{~nm}$ thick high $k$ dielectric $\mathrm{HfO}_{2}$ and was integrated with the device (See Figure 10c). Among the most important results obtained from this study are the relatively high mobility of SL $\mathrm{MoS}_{2}$ (close to $200 \mathrm{~cm}^{2} \mathrm{~V}^{-1} \mathrm{~s}^{-1}$ ) and the very high on-off ratio (larger than $1 \times 10^{8}$ ). These properties brought the TMDs to a competitive position when compared to other well-known electronic materials such as crystalline Silicon. Shortly after the first implementation of a single FET with monolayered $\mathrm{MoS}_{2}$, integrated circuit prototypes for analog and digital operations were successfully fabricated by the same methods [263, 264]. 


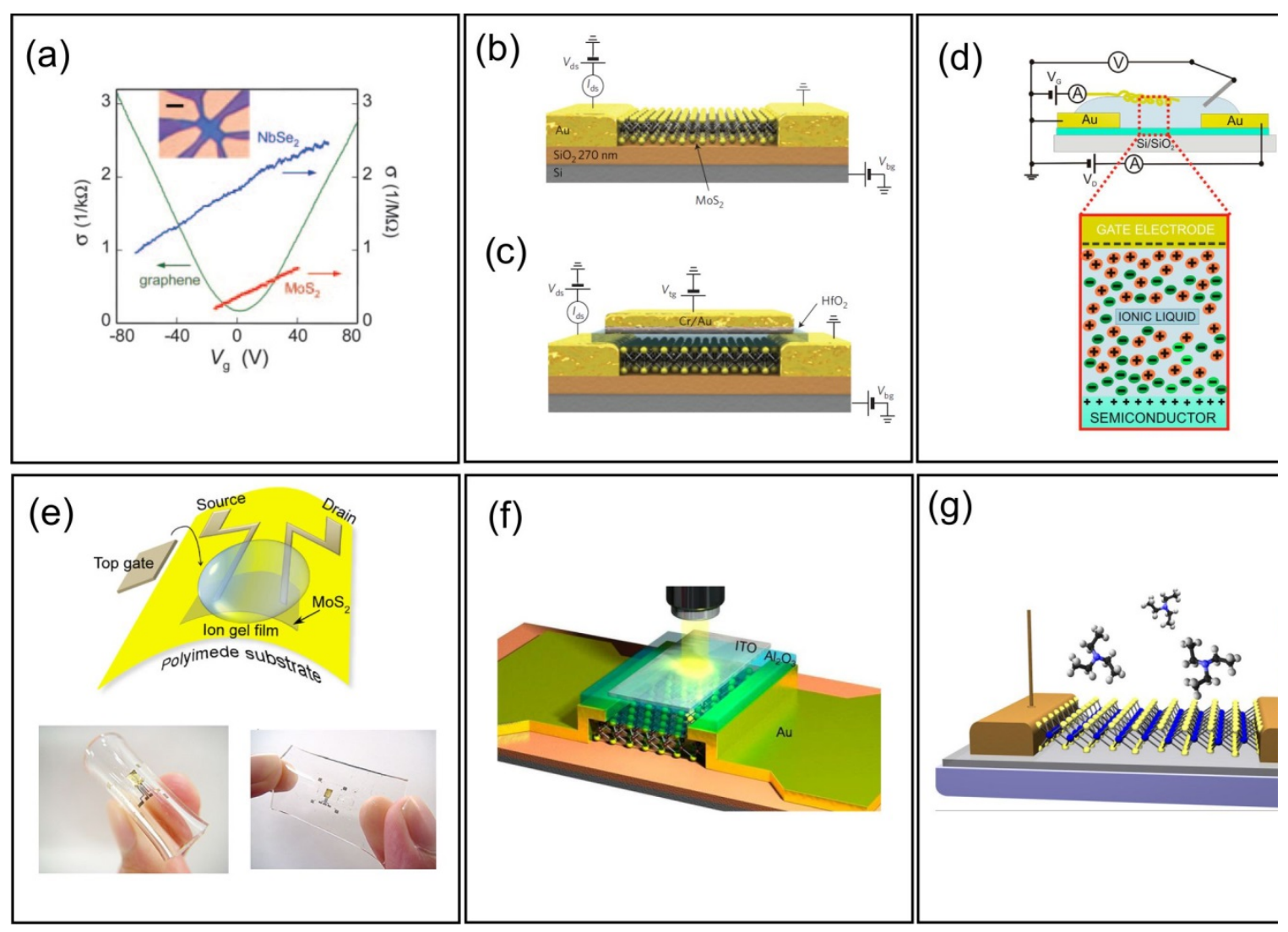

Figure 10 (a) Variation of the conductivity of single layer $\mathrm{MoS}_{2}$ and an optical image of the device used to measure this phenomenon. Reprinted with permission from Ref. [124]. Copyrights 2005 National Academy of Sciences. (b) Illustration of a FET with a bottom gate. (c) Illustration of a double gated FET where top and bottom gates are shown. Both (b) and (c) are reprinted with permission from Ref. [138]. Copyrights 2013 Nature Publishing Group. (d) Schematic representation of a Double Layer FEET top gated with an ionic liquid. Reprinted with permission from Ref. [205]. Copyrights 2012 American Chemical Society. (e) Schematic of a FET built on a flexible substrate. Reprinted with permission from Ref. [265]. Copyrights 2012 American Chemical Society. (f) Artistic rendering of a phototransistor device showing the light excitation source. Reprinted with permission from Ref. [259]. Copyrights 2012 American Chemical Society. (g) Schematic representation of a gas sensor device based on $\mathrm{MoS}_{2}$, where the sensed molecules are triethylamine (TEA). Reprinted with permission from Ref. [141]. Copyrights 2013 American Chemical Society.

Even though the physical and chemical properties of single layers of semiconducting TMDs seem almost ideal (i.e. ultra flat, free of dangling bonds, direct band gap), the materials are not rare and could be even inexpensive when compared to other electronic 
materials such as Indium or GaAs. Single layers of TMDs are not easy to obtain by the mechanical exfoliation (Scotch tape); this preparation method delivers samples formed by randomly oriented flakes in which the majority have more than one layer. Furthermore, it is unlikely to find a flake containing only one thickness throughout the flake. Because of this situation, various groups worldwide have explored the fabrication of devices with TMDs using few layered foils which are more abundant than mechanically exfoliated samples $[205,206,260,266]$. Alternatively, if the samples are prepared by CVD it is also possible to synthesize few layers and monolayers of TMDs $[78,156,260]$. As a result, a wide variety of devices have been fabricated with few-layered TMDs including FETs $[205,206]$, phototransistors [261, 267] and gas sensors [268].

A great deal of attention has been put into the characterization of the carrier mobility of TMDs. Table I summarizes the relevant studies that deal with the electronic properties of TMDs. 
Table 4 Survey of the reported carrier mobilities measured from electronic devices based on semiconducting TMDs

\begin{tabular}{|c|c|c|c|c|c|c|c|c|}
\hline Material & $\mathrm{N}$ of layers & $\begin{array}{l}\text { Synthesis } \\
\text { method }\end{array}$ & Device Type & $\begin{array}{l}\text { Electron } \mu \\
\left(\mathrm{cm}^{2} \mathrm{~V}^{-1} \mathrm{~s}^{-1}\right)\end{array}$ & $\begin{array}{c}\text { Hole } \boldsymbol{\mu} \\
\left(\mathrm{cm}^{2} \mathbf{V}^{-1} \mathbf{s}^{-1}\right)\end{array}$ & $\begin{array}{l}\text { On-OFF } \\
\text { ratio }\end{array}$ & Substrate & $\begin{array}{l}\text { Year and } \\
\text { Ref. }\end{array}$ \\
\hline $\mathrm{MoS}_{2}$ & 1 & $\begin{array}{l}\text { Mechanical } \\
\text { exfoliation }\end{array}$ & $\begin{array}{l}\text { FET, bottom and } \\
\text { top gated }\end{array}$ & 200 & ND & $1 \times 10^{8}$ & $\begin{array}{l}\mathrm{SiO}_{2} / \mathrm{Si} \\
(270 \mathrm{~nm})\end{array}$ & [89] \\
\hline $\mathrm{MoS}_{2}$ & 1 & CVD & $\begin{array}{l}\text { FET, Bottom and } \\
\text { top gated }\end{array}$ & $\begin{array}{l}21.6 \text { max } \\
\sim 13 \text { average }\end{array}$ & ND & ND & $\begin{array}{l}\mathrm{SiO}_{2} / \mathrm{Si} \\
(270 \mathrm{~nm})\end{array}$ & [269] \\
\hline $\mathrm{MoS}_{2}$ & 1 & CVD & FET, Bottom gated & 0.2 to 18 & ND & $6 \times 10^{6}$ & $\begin{array}{l}\mathrm{SiO}_{2} / \mathrm{Si} \\
(270 \mathrm{~nm})\end{array}$ & [163] \\
\hline $\mathrm{MoS}_{2}$ & 1 & $\begin{array}{l}\text { Mechanical } \\
\text { exfoliation }\end{array}$ & $\begin{array}{l}\text { FET, bottom and } \\
\text { top gated }\end{array}$ & $\begin{array}{l}\text { T dependent } \\
184 \text { Max }\end{array}$ & ND & $1 \times 10^{8}$ & $\begin{array}{l}\mathrm{SiO}_{2} / \mathrm{Si} \\
(270 \mathrm{~nm})\end{array}$ & [138] \\
\hline $\mathrm{MoS}_{2}$ & 1 & CVD & FET, bottom gated & 6 to 18 & ND & $1 \times 10^{9}$ & $\begin{array}{l}\mathrm{SiO}_{2} / \mathrm{Si} \\
(270 \mathrm{~nm})\end{array}$ & [270] \\
\hline $\mathrm{MoS}_{2}$ & 1, 2 and 3 & Mechanical & FET, top gated & 80,27 and 10 & ND & $1 \times 10^{7}$ & $\begin{array}{l}\mathrm{SiO}_{2} / \mathrm{Si} \\
(285 \mathrm{~nm})\end{array}$ & [259] \\
\hline $\mathrm{WSe}_{2}$ & 1 & CVD & $\begin{array}{l}\text { FET, top liquid } \\
\text { gated }\end{array}$ & 7 & 90 & ND & Sapphire & [215] \\
\hline $\mathrm{WSe}_{2}$ & 10 & $\begin{array}{l}\text { CVT } \quad \& \\
\text { mechanical } \\
\text { exfoliation }\end{array}$ & FET, bottom gated & $\begin{array}{l}650 @ 100 \mathrm{~K} \\
200 @ 300 \mathrm{~K}\end{array}$ & 90 & ND & $\mathrm{SiO}_{2}$ & [271] \\
\hline $\mathrm{MoS}_{2}$ & 2 or more & Wet Chemistry & FET, bottom gated & 6 & ND & $1 \times 10^{5}$ & $\begin{array}{l}\mathrm{SiO}_{2} / \mathrm{Si} \\
(300 \mathrm{~nm})\end{array}$ & [154] \\
\hline $\mathrm{MoS}_{2}$ & 2 or more & CVD & FET, top gated & 12.5 & ND & $1 \times 10^{5}$ & Polyimide & [265] \\
\hline $\mathrm{MoS}_{2}$ & $\begin{array}{ll}2 & \text { or } \\
\text { more }\end{array}$ & $\begin{array}{l}\text { Mechanical } \\
\text { exfoliation }\end{array}$ & FET, top gated & 44 & 86 & $1 \times 10^{2}$ & Sapphire & [205] \\
\hline $\mathrm{MoS}_{2}$ & $\sim 10$ & $\begin{array}{l}\text { Mechanical } \\
\text { exfoliation }\end{array}$ & FET, bottom gated & 184 & & ND & $\begin{array}{l}\mathrm{SiO}_{2} / \mathrm{Si} \\
(100 \mathrm{~nm})\end{array}$ & [206] \\
\hline $\mathrm{MoS}_{2}$ & $\sim 19$ & $\begin{array}{l}\text { Mechanical } \\
\text { exfoliation }\end{array}$ & $\begin{array}{l}\text { FET, bottom gated, } \\
2 \text { probe }\end{array}$ & 91 & ND & $1 \times 10^{6}$ & $\begin{array}{l}\mathrm{SiO}_{2} / \mathrm{Si} \\
(270 \mathrm{~nm})\end{array}$ & [272] \\
\hline $\mathrm{MoS}_{2}$ & $\sim 19$ & $\begin{array}{l}\text { Mechanical } \\
\text { exfoliation }\end{array}$ & $\begin{array}{l}\text { FET, bottom gated, } \\
4 \text { probe }\end{array}$ & 306.5 & ND & $1 \times 10^{6}$ & $\begin{array}{l}\mathrm{SiO}_{2} / \mathrm{Si} \\
(270 \mathrm{~nm})\end{array}$ & [272] \\
\hline $\mathbf{W S}_{2}$ & 30 to 90 & $\begin{array}{l}\text { Mechanical } \\
\text { exfoliation }\end{array}$ & $\begin{array}{l}\text { FET, top liquid } \\
\text { gated }\end{array}$ & $\sim 65$ & $\sim 105$ & ND & $\mathrm{SiO}_{2} / \mathrm{Si}$ & [273] \\
\hline WS $_{2}$ & $\sim 30$ & $\begin{array}{l}\text { Mechanical } \\
\text { exfoliation }\end{array}$ & FET, Bottom gated & ND & ND & $1 \times 10^{5}$ & $\mathrm{SiO}_{2} / \mathrm{Si}$ & [260] \\
\hline
\end{tabular}

From the data shown in Table 4, it can be easily concluded that $\mathrm{MoS}_{2}$ is the most studied material among the semiconducting TMDs, and others remain to be fully investigated. In addition, $\mathrm{MoS}_{2}$ is the only one that has so far allowed fabrication of transistors with monolayer flakes. Other materials such as $\mathrm{WS}_{2}$ has been used, but in the few-layered form. Some attempts to use $\mathrm{WS}_{2}$ single layers have been made but the contact resistance has been extremely high usually in the range of G $\Omega$ [156]. Another characteristic fact is that the mobility in $\mathrm{MoS}_{2}$ appears to strongly depend on the device 
geometry and can vary considerably from one device to the next [269]. In this matter, Balicas et al. found that for few-layer $\mathrm{MoS}_{2}$ the two-terminal configuration underestimates the intrinsic field effect mobility of $\mathrm{MoS}_{2}$ on $\mathrm{SiO}_{2}$; the authors found that four probe measurements were more accurate [272]. They also analyzed the performance of mechanically exfoliated $\mathrm{MoTe}_{2}$ based FET and were able to obtain $\mathrm{I}_{\mathrm{ON}} / \mathrm{I}_{\mathrm{OFF}}$ ratios in the range similar to that of $\mathrm{MoS}_{2}$ and $\mathrm{MoSe}_{2}$ on $\mathrm{SiO}_{2}$. However, the material behaved as a hole-doped semiconductor in comparison with the electron-doped $\mathrm{MoS}_{2}$ [274].

\subsection{Optoelectronic devices}

Because of the match between the visible spectrum and the direct band gap energy of semiconducting single-layer TMDs, the use of these materials in the fabrication of optoelectronic devices is straightforward. The first report of a light sensitive device based on a monolayered TMD was carried out by Yin Z. Y. et al., in which the authors used a mechanically exfoliated $\mathrm{MoS}_{2}$ flake to build a phototransistor [258]. In the beginning, TMD phototransistors were not very sensitive and were capable of achieving photoresponsivities in the range of few $\mathrm{mA} / \mathrm{W}$. However, the tremendous research activity in TMD photosensors has recently lead to the achievement of very high responsivities, six orders of magnitude larger in just a couple of years [139]. It is clear that TMDs could be implemented in very small detectors at device densities beyond what has been achieved for conventional silicon technology. Additionally, TMDs

photodetectors exhibit some grade of band gap modulation as additional layers are added to the single layer device $[79,259]$.

Furthermore, TMDs can also be used in photovoltaic conversion devices. DFT 
calculations have demonstrated monolayer TMDs (e.g. $\mathrm{MoS}_{2}, \mathrm{MoSe}_{2}, \mathrm{WS}_{2}$ ) can absorb up to $5-10 \%$ of the incident sunlight, which is one order of magnitude higher than that of Si and GaAs [275]. It has been experimentally demonstrated that by stacking different layered materials (TMDs, graphene, etc) can lead to the development of ultrasensitive light detectors [139], or flexible energy harvesting devices [262, 276]. Very recently, it is demonstrated that TMDs can serve as an interfacial layer to improve the power conversion efficiency (PCE) of graphene/silicon solar cells [277, 278]. TMDs (e.g. $\mathrm{MoS}_{2}$ ) can act as both an effective passivation layer and electron-blocking/hole-transporting layer and lead to a PCE up to $11.1 \%$ [278]. This may open up new avenues for developing high-efficiency and cost-effective inorganic solar cells.

\subsection{Other devices}

Besides FETs and photosensors, TMDs have been used to build devices for other purposes, such as gas sensors [141, 268, 279], electron emission sources [280], memory devices [281, 282], light emitting sources by electroluminescence [283] and p-n junctions [257]. In addition, it was proposed that TMDs have intrinsic piezoelectricity which could allow them to be strain sensing elements and actuators in nanoscale devices [284]. Experimental evidence of piezoelectricity has been recently reported for SL-MoS 2 [285]. TMDs are also suitable candidates for ultrashort optical pulse characterization as demonstrated by Janisch et al. in a recent study [286]. Exfoliated TMDs also exhibit better electrochemical storage capacities in comparison with their bulk counterparts as reported by Winchester et al., thus opening up a less explored avenue for these materials [287]. 


\section{Defect engineering of TMDs}

Similar to 3-D crystals, few layered TMDs possess defects which play a role in changing their opto-electronic properties. Due to the 2-D nature of TMD monolayers, defects can also be categorized similarly as in graphene [7]: vacancies, topological defects (grain boundaries and extended line of defects), edge effects and functionalization in nanoribbons, and defects that change the curvature of the structure (inorganic fullerenes, nanotube caps, etc.,) $[48,288,289]$. In addition, defects associated with doping or alloying and different stackings can be added to the types of defects found in TMD. This section is devoted to study the properties of defective sheets of TMD and their importance in tuning the band gap in semiconducting TMDs.

\subsection{Vacancies}

The use of aberration corrected high-resolution transmission electron microscopes (AC-HRTEM) has made possible to identify and even generate, with the electron beam, sulfur vacancies in monolayered $\mathrm{MoS}_{2}$ [290]. Since in the trigonal prismatic TMD structure of the type $\mathrm{MX}_{2}(\mathrm{M}=$ transition metal, $\mathrm{X}=$ challcogen atom $)$ there are two layers of chalcogen atoms, there can be single $\left(\mathrm{V}_{\mathrm{x}}\right)$ and double vacancies $\left(\mathrm{V}_{\mathrm{x} 2}\right)$ if one or two sulfurs are removed (see Figure 11a). In addition, vacancies involving the absence of the transition metal atom are also possible $\left(\mathrm{V}_{\mathrm{M}}\right)$. Moreover, anti-site defects in which the transition metal atom substitutes a column of two chalcogen atoms denoted as $\mathrm{M}_{\mathrm{x} 2}$ can be produced [291]. Similarly, the transition metal atom can be substituted by two sulfurs referred as $\mathrm{X} 2_{\mathrm{M}}$ [291]. Since vacancies make more reactive the TMD layers, they might 
also play an important role for doping. Yakobson's group has studied the stability of these vacancy defects using DFT, and they showed that $V_{x}$ type single vacancy exhibits the lower formation energy and the anti-site defects possess the higher formation energies; this result agrees with experimental observations [291].

\subsection{Topological defects: grain boundaries in TMD}

Through chemical vapor deposition (CVD) techniques, in which the TMD starts growing by triangular clusters, the clusters get bigger and coalesce forming grain boundaries with different structures and properties (see Figure 11b-f) [162, 163, 292]. Ajayan's group reported grain boundaries in monolayer $\mathrm{MoS}_{2}$ obtained by CVD composed of heptagonal-pentagonal dislocations (Figure 11b-d), and Yakobson's group described them using first principles calculations showing that different grain boundaries introduce mid-gap electronic states acting as sinks for carriers. In particular, the $60^{\circ}$ grain boundary which bisects two misoriented $\mathrm{MoS}_{2}$ domains exhibits metallic behavior along the boundary [291, 293]. In the same context, van der Zande et al., found, also using a CVD technique, grain boundaries containing square-octagon-like defects which produce crystal tilt and mirror boundaries exhibiting also mid-gap localized states as in the case of heptagon-pentagon-like defects [162] (Figure 11e-f). Interestingly, the crystal tilt boundary revealed an enhanced PL, while the mirror boundary showed a quenched PL. Defect dynamics at the grain boundary in TMD has been studied extensively using HRTEM by Azizi et al in a recent report. Very interesting observations have been made about the migration of dislocation as well as the strain fields they cause at the grain boundaries of monolayer $\mathrm{WS}_{2}$ [294]. 

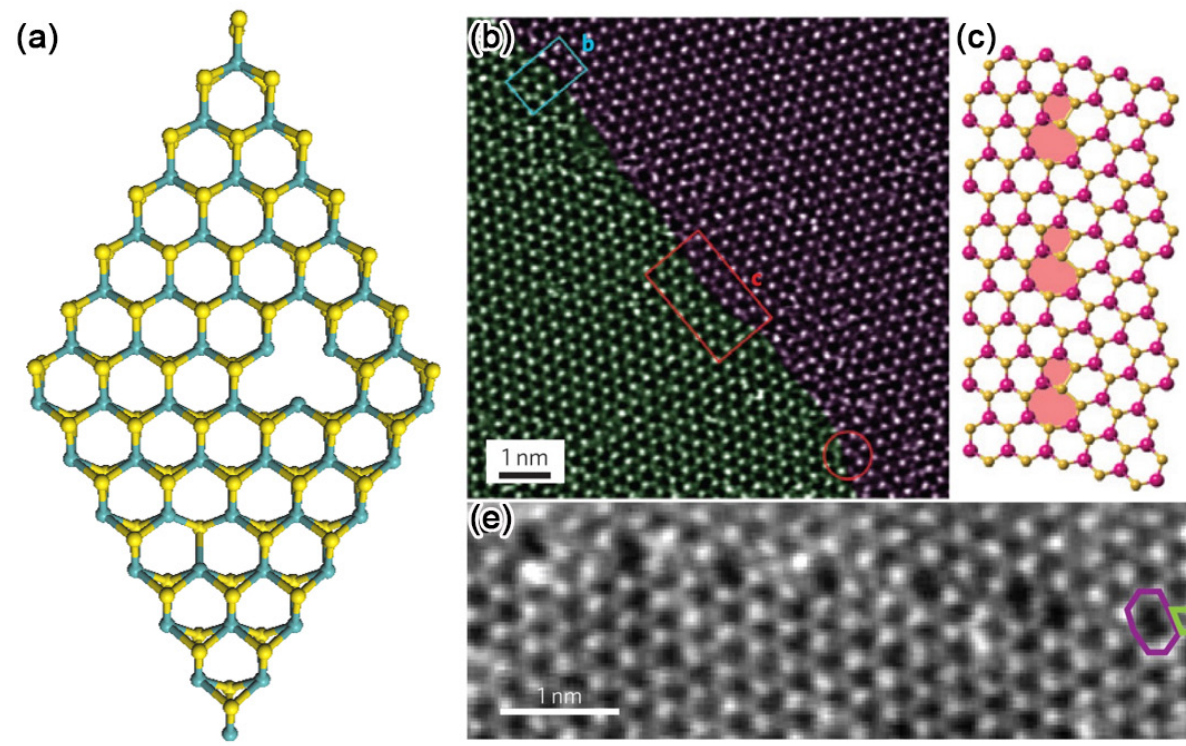

(d) $\mathrm{M}$

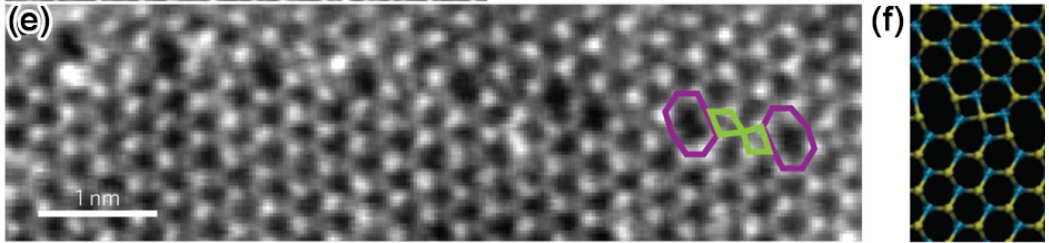

Figure 11 Sulfur vacancies and grain boundaries in $\mathrm{MoS}_{2}$ (a) Model of monolayer $\mathrm{MoS}_{2}$ showing a double sulfur vacancy $\left(\mathrm{V}_{\mathrm{s} 2}\right.$. Black circle) and a monovacancy $\left(\mathrm{V}_{\mathrm{s}}\right.$. Red circle). (b) AC-HRTEM micrograph of a grain boundary made of pentagonal-heptagonal dislocation defects. (c) and (d) Models of the pentagon-heptagon defects and different orientations showing the top and bottom chalcogen atoms (yellow top atoms and orange down atoms. ((b-d) are reprinted with permission from Ref. [163]. Copyrights 2013 Nature Publishing Group). (e) Observation of a grain boundary composed of 8-4 defects. (f) Model of (e). Reprinted with permission from Ref. [162]. Copyrights 2013 Nature Publishing Group).

\subsection{Doping and alloying}

Since single $V_{x}$ vacancies exhibit smaller formation energies than the other point defects, it is natural to think that these could be filled by another type of atom which can be another chalcogen such as Se or Te, thus preserving the trigonal prismatic arrangement, but with different bond lengths. DFT-LDA calculations on $\mathrm{MoS}_{2}$ show that when all the sulfur atoms in the upper layer are replaced by selenium (see Figure 12a-b), the band gap continues being direct at the $\mathrm{K}$ point with a magnitude of $1.71 \mathrm{eV}$, which is the average value of the gaps for $\mathrm{MoS}_{2}(1.82 \mathrm{eV})$ and $\mathrm{MoSe}_{2}(1.60 \mathrm{eV})$. Therefore, the band gap can 
be easily tuned when exchanging the chalcogen atoms. Recently, using CVD, an alloy of the type $\mathrm{MoS}_{2 \mathrm{x}} \mathrm{Se}_{2(1-\mathrm{x})}$ was reported [212]; in this case DFT-LDA calculations show a direct band gap with a linear decrease from $1.82 \mathrm{eV}$ to $1.6 \mathrm{eV}$. Another possibility is to from alloys by substituting the transition metal atom $\mathrm{M}_{\mathrm{ay}} \mathrm{M}_{\mathrm{b}(1-\mathrm{y})} \mathrm{X}_{2}$. Using chemical vapor transport techniques with Bromine, 3-D crystals with compositions $\mathrm{Mo}_{\mathrm{y}} \mathrm{W}_{(1-\mathrm{y})} \mathrm{S}_{2}$ have been obtained and their 2-D layers have been isolated by adhesive tape exfoliation [137, 295]. PL measurements on different concentrations of these systems show that the gap can be tuned from $1.82 \mathrm{eV}(\mathrm{y}=0.8)$ to $1.99 \mathrm{eV}(\mathrm{y}=0)$ [137]. Still there is a lot of work to do in this area. For example, doping or alloying with $\mathrm{Nb}$ or $\mathrm{Re}$ could provide unprecedented properties since $\mathrm{NbS}_{2}$ and $\mathrm{NbSe}_{2}$ are trigonal prismatic metals and $\mathrm{ReS}_{2}$ is a semiconductor with distorted octahedral structure.

\subsection{Different stackings, heterostructures and van der Waals}

\section{Solids}

It has been mentioned in this account that as the number of layers in semiconducting TMDs are reduced, the indirect band gap increases and at the monolayer limit, the layer becomes a direct band gap semiconductor. This behavior opens fascinating possibilities when studying few-layered systems since they possess different electronic properties from the bulk and from the monolayer (see Figure 12c). Recently, transistors fabricated from $\mathrm{MoS}_{2}$ bilayers have been built [296]. In addition, it has been found using DFT-LDA calculations that in bilayers of $\mathrm{WS}_{2}, \mathrm{WSe} 2, \mathrm{MoS}_{2}$ and $\mathrm{MoSe}_{2}$, the indirect and direct band gaps can become very close to one another with differences of around $0.14 \mathrm{eV}$, depending on the stacking order, nevertheless, the indirect band gap always dominates 
[109]. However, if different semiconducting TMDs are used to form heterobilayers, band gap tuning and direct band gaps might be possible (see Figure 12d-h) [109, 266, 297, 298]. Another interesting possibility that has been studied is a heterobilayer out of a semiconductor $\left(\mathrm{MoS}_{2}, \mathrm{WSe}_{2}, \mathrm{WS}_{2}, \mathrm{MoSe}_{2}\right)$ with a metal such as $\mathrm{NbS}_{2}$ or $\mathrm{NbSe}_{2}$ [109]. For these cases, metallic systems are obtained with a characteristic band structure above the Fermi level in which the bands almost touch in one point (at the K point). Further experimental studies along this direction need to be carried out.

(a)

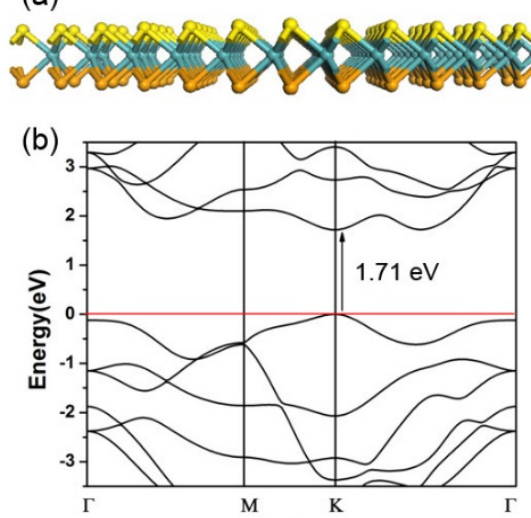

(d)
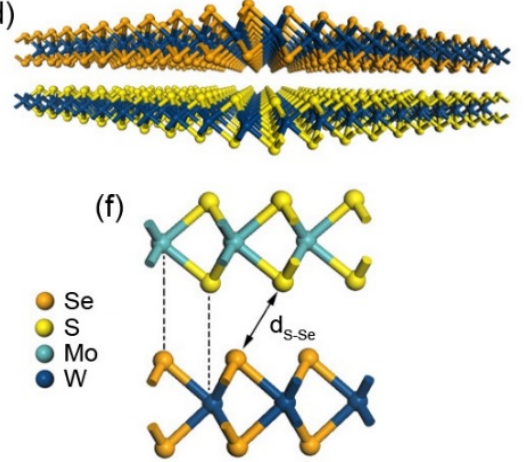

(c) $\mathrm{WSe}_{2}$ Monolayer

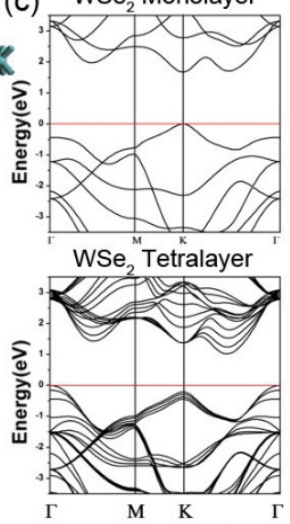

(e)

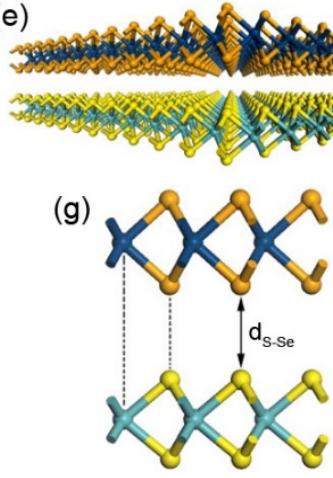

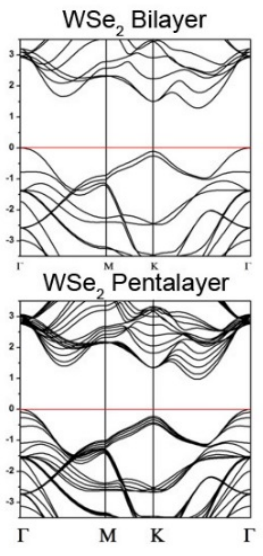

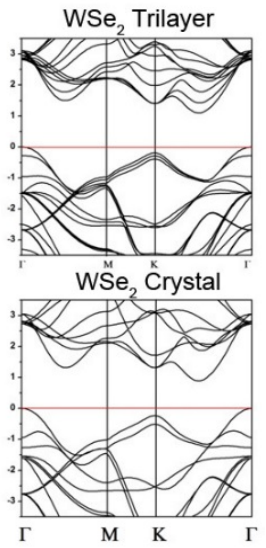

( $\mathrm{h}$

(h) Bilayer $\left(\mathrm{WS}_{2}, \mathrm{NbSe}_{2}, \mathrm{~A}\right)$

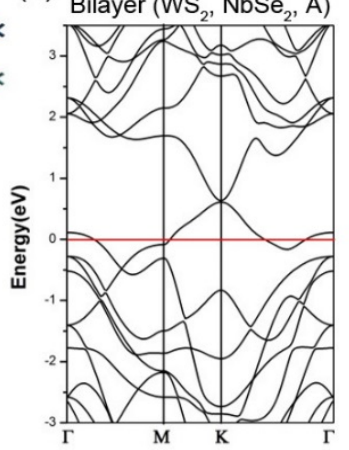

Figure 12 (a) Computer model of MoSSe alloy: top yellow atoms are sulfur, bottom orange atoms are selenium. (b) DFT-LDA band structure of MoSSe alloy (the red line is at the top of the valence band). (c) DFT-LDA band structures of $W e_{2}$ from $L=1$ to $L=5$ and bulk (the red line is at the top of the valence band. (d-e) Heterobilayers of TMD with two different stackings. (f) Stacking type B (bulk). (g) Stacking type A. $d_{S-S e}$ is the distance between chalcogen atoms of adjacent layers. Reprinted with permission from Ref. [109]. Copyrights 2013 Nature Publishing Group. (h) Band structure of a heterobilayer made out of $\mathrm{WS}_{2}$ and $\mathrm{NbSe}_{2}$. 


\subsection{Edge effects in TMD nanoribbons}

Motivated by the edge effects in graphene nanoribbons [7, 299-301], researchers started to shed light in the behavior of edges in semiconducting TMD nanoribbons. It has been reported experimentally that triangular clusters exhibiting zig-zag edge terminations of $\mathrm{MoS}_{2}$ exhibit metallic behavior, exactly at the edges, thus forming a nanowire embedded in a semiconductor [302]. This result has been confirmed theoretically by several authors using first principles calculations based on density functional theory (DFT), adding also the possibility of having ferromagnetic states for the zig-zag configuration [62-64]. Armchair edges exhibit semiconducting behavior when saturating the edges with hydrogen [62-64]. Interestingly, $\mathrm{WS}_{2}$ triangular clusters grown by the CVD method, possess a huge PL response at the edges [76] which is caused by the edge passivation. Certainly functionalizing edge states with different atoms at the edges of TMDs is going to provide different responses depending on the atom used. One of the challenges which needs to be addressed is to gain more control during the synthesis process for selective edge passivation and the generation of nanorribbons with the desired edge geometry.

\subsection{Chemical functionalization of TMDs by defect engineering}

In order to tailor the surface properties, the functionalization of TMD nanosheets is necessary to improve their dispersion in common solvents (e.g. acetone, isopropanol (IPA)) or affinity to some biomolecules (e.g. proteins). However, it is still a challenge to functionalize TMDs due to their relative chemical inertness. If some defects can be 
created in TMD nanosheets, it will be possible to improve the surface activity of TMDs and lead to the grafting of some chemical groups. Chou et al. demonstrated the ligand conjugation of chemically exfoliated $\mathrm{MoS}_{2}$ nanosheets [303]. Due to the violent nature during chemical exfoliation, many defects, including internal edges and perimeter edges, will be generated and play important roles in improving the ligand modifications. In this manner, it is possible to tune the surface functionality of TMD nanosheets and enable their bio-applications, such as in artificial receptors for enzymes [303]. Very recently, Backes et al. found that the reaction of liquid-exfoliated 2D $\mathrm{MoS}_{2}$ nanosheets with $\mathrm{M}(\mathrm{OAc})_{2}$ slats $(\mathrm{M}=\mathrm{Ni}, \mathrm{Cu}, \mathrm{Zn}$; OAc=acetate $)$ can yield functionalized $\mathrm{MoS}_{2}-\mathrm{M}(\mathrm{OAc})_{2}$, which can lead to a nice dispersion of TMD nanosheets in more conventional laboratory solvents (e.g. acetone, IPA) besides N-methyl-2-pyrrolidone (NMP) [304]. This actually provides a facile route for the functionalization of TMD nanosheets and facilitates their applications. It is worthy to point out that the research progress on chemical functionalization on 2D TMDs is still very scarce so far. More efforts need to be paid along this direction to boost the possible applications of TMDs in a broader fields.

\section{Conclusions and perspectives}

Layered materials have received an explosive increase in attention in recent years since the work of Novoselov and Geim on graphene. Despite the many wonderful properties, one application area not accessible to graphene is aspects of optoelectronics where the absence of a bandgap greatly limits the scope of graphene applications. For this reason, researchers have been attracted to layered materials which do have a bandgap that is suitable for optoelectronic applications. In this article we have shown that TMDs are 
layered compounds with a variety of unique properties that are interesting from the viewpoint of basic science as well as for the special potential for applications. These properties are quite different from the properties of silicon, which has been the workhorse of the information revolution taking place during the past 50 or more years in accordance with the Moore's Law schedule.

Graphene, has a set of wonderful properties in its own right, that are also different from those of silicon. But it is already clear to most people that the absence of a bangap precludes its usage as a silicon replacement. But TMDs do possess a band gap and can be used in optoelectronics. This point has of course been emphasized in this article, but we stress here in the summary that we should not think of TMD materials as a replacement for silicon nor as a replacement for graphene.

TMDs are instead a materials class with many highly desirable properties in addition to having a bandgap. The transition metal species can be changed to make a semiconductor or a metal, a material with a variety of magnetic properties ranging from a ferromagnet, to an antiferromagnet, or a paramagnet. These materials can also be superconducting or not. Changing the chalcogen species can be used for smaller property changes than changing the transition metal species. They can also be prepared in bulk form, in layered form, in wire form and as a quantum dot, with different optical, electronic and other properties. For all these reasons, we should think of TMDs as an interesting class of materials now under intense investigation for their own potential to form fascinating materials with their own special properties that can be exploited for many unprecedented applications with a potential for high societal impact. 


\section{Acknowledgments}

This work is supported by the U.S. Army Research Office MURI grant

W911NF-11-1-0362. R.L. acknowledges the support from the National Natural Science Foundation of China (Grant No. 51372131) and the National Basic Research Program of China (2014CB932401, 2015CB932500). M.T. also acknowledges support from the National Science Foundation EFRI-2DARE project \# 1542707. H.R.G. acknowledges support from National Science Foundation (DMR-1454240). M.S.D. acknowledges support from NSF (DHR-1004147). The authors also acknowledge the Center for 2-Dimensional and Layered Materials.

\section{References}

[1] G.H. Gao, W. Gao, E. Cannuccia, J. Taha-Tijerina, L. Balicas, A. Mathkar, T.N. Narayanan, Z. Liu, B.K. Gupta, J. Peng, Y.S. Yin, A. Rubio, P.M. Ajayan, Nano Lett., 12 (2012) 3518.

[2] K.S. Novoselov, Z. Jiang, Y. Zhang, S.V. Morozov, H.L. Stormer, U. Zeitler, J.C. Maan, G.S. Boebinger, P. Kim, A.K. Geim, Science, 315 (2007) 1379.

[3] A.K. Geim, K.S. Novoselov, Nat. Mater., 6 (2007) 183.

[4] W.W. Cai, A.L. Moore, Y.W. Zhu, X.S. Li, S.S. Chen, L. Shi, R.S. Ruoff, Nano Lett., $10(2010) 1645$.

[5] A.A. Balandin, S. Ghosh, W.Z. Bao, I. Calizo, D. Teweldebrhan, F. Miao, C.N. Lau, Nano Lett., 8 (2008) 902.

[6] C. Lee, X.D. Wei, J.W. Kysar, J. Hone, Science, 321 (2008) 385.

[7] H. Terrones, R.T. Lv, M. Terrones, M.S. Dresselhaus, Rep. Prog. Phys., 75 (2012) 062501.

[8] R.T. Lv, M. Terrones, Mater. Lett., 78 (2012) 209.

[9] R. Mas-Balleste, C. Gomez-Navarro, J. Gomez-Herrero, F. Zamora, Nanoscale, 3 (2011) 20.

[10] Q.H. Wang, K. Kalantar-Zadeh, A. Kis, J.N. Coleman, M.S. Strano, Nat. Nanotechnol., 7 (2012) 699.

[11] M. Osada, T. Sasaki, Adv. Mater., 24 (2012) 210.

[12] M. Chhowalla, H.S. Shin, G. Eda, L.J. Li, K.P. Loh, H. Zhang, Nat. Chem., 5 (2013) 263.

[13] X.F. Song, J.L. Hu, H.B. Zeng, J. Mater. Chem. C, 1 (2013) 2952. 
[14] S.Z. Butler, S.M. Hollen, L.Y. Cao, Y. Cui, J.A. Gupta, H.R. Gutierrez, T.F. Heinz, S.S. Hong, J.X. Huang, A.F. Ismach, E. Johnston-Halperin, M. Kuno, V.V. Plashnitsa, R.D. Robinson, R.S. Ruoff, S. Salahuddin, J. Shan, L. Shi, M.G. Spencer, M. Terrones, W. Windl, J.E. Goldberger, ACS Nano, 7 (2013) 2898.

[15] Q. Tang, Z. Zhou, Prog. Mater. Sci., 58 (2013) 1244.

[16] M. Xu, T. Liang, M. Shi, H. Chen, Chem. Rev., 113 (2013) 3766.

[17] X. Huang, Z.Y. Zeng, H. Zhang, Chem. Soc. Rev., 42 (2013) 1934.

[18] A.K. Geim, I.V. Grigorieva, Nature, 499 (2013) 419.

[19] C. Tan, H. Zhang, Chem. Soc. Rev., 44 (2015) 2713.

[20] C. Tan, Z. Liu, W. Huang, H. Zhang, Chem. Soc. Rev., 44 (2015) 2615.

[21] Y. Chen, C. Tan, H. Zhang, L. Wang, Chem. Soc. Rev., 44 (2015) 2681.

[22] H. Li, J. Wu, Z. Yin, H. Zhang, Acc. Chem. Res., 47 (2014) 1067.

[23] X. Huang, C. Tan, Z. Yin, H. Zhang, Adv. Mater., 26 (2014) 2185.

[24] R. Lv, J.A. Robinson, R.E. Schaak, D. Sun, Y.F. Sun, T.E. Mallouk, M. Terrones, Acc. Chem. Res., 48 (2015) 56.

[25] R.F. Frindt, Phys. Rev., 140 (1965) 536.

[26] H. Tributsch, Berichte der Bunsengesellschaft für physikalische Chemie, 81 (1977) 361.

[27] H.J. Lewerenz, A. Heller, F.J. DiSalvo, J. Am. Chem. Soc., 102 (1980) 1877.

[28] H. Tributsch, Faraday Discuss. Chem. Soc., 70 (1980) 189.

[29] R. Audas, J.C. Irwin, J. Appl. Phys., 52 (1981) 6954.

[30] G. Razzini, L.P. Bicelli, G. Pini, B. Scrosati, J Electrochem Soc, 128 (1981) 2134.

[31] B. Parkinson, Acc. Chem. Res., 17 (1984) 431.

[32] G. Kline, K.K. Kam, R. Ziegler, B.A. Parkinson, Solar Energy Mater., 6 (1982) 337.

[33] J. Chen, X.-J. Wu, L. Yin, B. Li, X. Hong, Z. Fan, B. Chen, C. Xue, H. Zhang, Angew. Chem. Int. Ed., 54 (2015) 1210.

[34] L.H. Brixner, J. Inorg. Nuclear Chem., 24 (1962) 257.

[35] T.C. Holgate, Y. Liu, D. Hitchcock, T.M. Tritt, J. He, J. Electronic Mater., 42 (2013) 1751.

[36] L.H. Brixner, G. Teufer, Inorg. Chem., 2 (1963) 992.

[37] R. Mansfield, S.A. Salam, Proc. Phys. Soc. B, 66 (1953) 677.

[38] H.J. Goldsmid, Proc. Phys. Soc. London B, 67 (1954) 360.

[39] H.J. Goldsmid, R.W. Douglas, British J. Appl. Phys., 5 (1954) 386.

[40] J.A. Champion, British J. Appl. Phys., 16 (1965) 1035.

[41] E. Revolinsky, D. Beerntsen, J. Appl. Phys., 35 (1964) 2086.

[42] W.T. Hicks, J Electrochem Soc, 111 (1964) 1058.

[43] A. Conan, G. Goureaux, M. Zoaeter, J. Phys. Chem. Solids, 36 (1975) 315.

[44] C. Chiritescu, D.G. Cahill, N. Nguyen, D. Johnson, A. Bodapati, P. Keblinski, P. Zschack, Science, 315 (2007) 351.

[45] L.H. Hihara, R.M. Latanision, Corrosion, 48 (1992) 546.

[46] R.H. Savage, J. Appl. Phys., 19 (1948) 1.

[47] B.K. Yen, B.E. Schwickert, M.F. Toney, Appl. Phys. Lett., 84 (2004) 4702.

[48] R. Tenne, L. Margulis, M. Genut, G. Hodes, Nature, 360 (1992) 444.

[49] M.T. Lavik, T.M. Medved, G.D. Moore, A S L E Transactions, 11 (1968) 44.

[50] S.V. Prasad, J.S. Zabinski, J. Mater. Sci. Lett., 12 (1993) 1413.

[51] V.R. Johnson, G.W. Vaughn, J. Appl. Phys., 27 (1956) 1173. 
[52] V.R. Johnson, M.T. Lavik, G.W. Vaughn, J. Appl. Phys., 28 (1957) 821.

[53] F. McTaggart, A. Moore, Aust. J. Chem., 11 (1958) 481.

[54] J.M. Martin, C. Donnet, T. Le Mogne, T. Epicier, Phys. Rev. B, 48 (1993) 10583.

[55] S. Watanabe, J. Noshiro, S. Miyake, Surface Coatings Technol., 183 (2004) 347.

[56] X. Ding, X.T. Zeng, X.Y. He, Z. Chen, Surface Coatings Technol., 205 (2010) 224.

[57] D.G. Teer, Wear, 251 (2001) 1068.

[58] M.R. Hilton, R. Bauer, S.V. Didziulis, M.T. Dugger, J.M. Keem, J. Scholhamer, Surface Coatings Technol., 53 (1992) 13.

[59] G. Eda, H. Yamaguchi, D. Voiry, T. Fujita, M.W. Chen, M. Chhowalla, Nano Lett., 11 (2011) 5111.

[60] K.F. Mak, C. Lee, J. Hone, J. Shan, T.F. Heinz, Phys. Rev. Lett., 105 (2010) 136805.

[61] A. Splendiani, L. Sun, Y.B. Zhang, T.S. Li, J. Kim, C.Y. Chim, G. Galli, F. Wang, Nano Lett., 10 (2010) 1271.

[62] A.R. Botello-Mendez, F. Lopez-Urias, M. Terrones, H. Terrones, Nanotechnology, 20 (2009) 325703.

[63] J. Zhang, J.M. Soon, K.P. Loh, J.H. Yin, J. Ding, M.B. Sullivian, P. Wu, Nano Lett., 7 (2007) 2370.

[64] C. Ataca, H. Sahin, E. Akturk, S. Ciraci, J. Phys. Chem. C, 115 (2011) 3934.

[65] R.E. Jones, H.R. Shanks, B. Morosin, Finnemor.Dk, Phys. Rev. B, 6 (1972) 835.

[66] I. Guillamon, H. Suderow, S. Vieira, L. Cario, P. Diener, P. Rodiere, Phys. Rev. Lett., 101 (2008) 4.

[67] V.G. Tissen, M.R. Osorio, J.P. Brison, N.M. Nemes, M. Garcia-Hernandez, L. Cario,

P. Rodiere, S. Vieira, H. Suderow, Phys. Rev. B, 87 (2013) 6.

[68] R.F. Frindt, J. Appl. Phys., 37 (1966) 1928.

[69] P. Joensen, R.F. Frindt, S.R. Morrison, Mater. Res. Bull., 21 (1986) 457.

[70] Consadori.F, R.F. Frindt, Phys. Rev. B, 2 (1970) 4893.

[71] H. Terrones, M. Terrones, J. Mater. Res., 29 (2014) 373.

[72] R. Coehoorn, C. Haas, R.A. Degroot, Phys. Rev. B, 35 (1987) 6203.

[73] R. Coehoorn, C. Haas, J. Dijkstra, C.J.F. Flipse, R.A. Degroot, A. Wold, Phys. Rev. B, 35 (1987) 6195.

[74] V. Nicolosi, M. Chhowalla, M.G. Kanatzidis, M.S. Strano, J.N. Coleman, Science, 340 (2013) DOI: 10.1126/science.1226419.

[75] R. Tenne, L. Margulis, G. Hodes, Adv. Mater., 5 (1993) 386.

[76] H.R. Gutiérrez, N. Perea-López, A.L. Elías, A. Berkdemir, B. Wang, R. Lv, F. López-Urías, V.H. Crespi, H. Terrones, T. M., Nano Lett., 13 (2013) 3447.

[77] W.J. Zhao, Z. Ghorannevis, L.Q. Chu, M.L. Toh, C. Kloc, P.H. Tan, G. Eda, ACS Nano, 7 (2013) 791.

[78] A.L. Elias, N. Perea-Lopez, A. Castro-Beltran, A. Berkdemir, R.T. Lv, S.M. Feng, A.D. Long, T. Hayashi, Y.A. Kim, M. Endo, H.R. Gutierrez, N.R. Pradhan, L. Balicas, T.E.M. Houk, F. Lopez-Urias, H. Terrones, M. Terrones, ACS Nano, 7 (2013) 5235.

[79] A. Berkdemir, H.R. Gutierrez, A.R. Botello-Mendez, N. Perea-Lopez, A.L. Elias, C.I. Chia, B. Wang, V.H. Crespi, F. Lopez-Urias, J.C. Charlier, H. Terrones, M. Terrones, Sci. Rep., 3 (2013) 1755.

[80] T.J. Wieting, J.L. Verble, Phys. Rev. B, 3 (1971) 4286.

[81] D.G. Mead, J.C. Irwin, Can. J. Phys., 55 (1977) 379. 
[82] T. Sekine, M. Izumi, T. Nakashizu, K. Uchinokura, E. Matsuura, J. Phys. Soc. Jpn., 49 (1980) 1069.

[83] H. Li, G. Lu, Y.L. Wang, Z.Y. Yin, C.X. Cong, Q.Y. He, L. Wang, F. Ding, T. Yu, H. Zhang, Small, 9 (2013) 1974.

[84] W. Zhao, Z. Ghorannevis, A.K. Kumar, J.R. Pang, M. Toh, X. Zhang, C. Kloc, P. Tan, G. Eda, Nanoscale, 5 (2013) 9677.

[85] H. Li, Q. Zhang, C.C.R. Yap, B.K. Tay, T.H.T. Edwin, A. Olivier, D. Baillargeat, Adv. Funct. Mater., 22 (2012) 1385.

[86] P. Tonndorf, R. Schmidt, P. Bottger, X. Zhang, J. Borner, A. Liebig, M. Albrecht, C. Kloc, O. Gordan, D.R.T. Zahn, S.M. de Vasconcellos, R. Bratschitsch, Opt. Express, 21 (2013) 4908.

[87] S. Tongay, J. Zhou, C. Ataca, K. Lo, T.S. Matthews, J.B. Li, J.C. Grossman, J.Q. Wu, Nano Lett., 12 (2012) 5576.

[88] A. Ayari, E. Cobas, O. Ogundadegbe, M.S. Fuhrer, J. Appl. Phys., 101 (2007) 014507.

[89] B. Radisavljevic, A. Radenovic, J. Brivio, V. Giacometti, A. Kis, Nat. Nanotechnol., $6(2011) 147$.

[90] K.F. Mak, K.L. He, C. Lee, G.H. Lee, J. Hone, T.F. Heinz, J. Shan, Nat. Mater., 12 (2013) 207.

[91] S. Tongay, J. Zhou, C. Ataca, J. Liu, J.S. Kang, T.S. Matthews, L. You, J.B. Li, J.C. Grossman, J.Q. Wu, Nano Lett., 13 (2013) 2831.

[92] V. Petkov, S.J.L. Billinge, J. Heising, M.G. Kanatzidis, J. Am. Chem. Soc., 122 (2000) 11571.

[93] X. Zhou, D. Yang, R.F. Frindt, J. Phys. Chem. Solids, 57 (1996) 1137.

[94] R. Bissessur, M.G. Kanatzidis, J.L. Schindler, C.R. Kannewurf, Journal of the Chemical Society-Chemical Communications, (1993) 1582.

[95] F. Wypych, R. Schollhorn, Journal of the Chemical Society-Chemical Communications, (1992) 1386.

[96] D. Yang, S.J. Sandoval, W.M.R. Divigalpitiya, J.C. Irwin, R.F. Frindt, Phys. Rev. B, 43 (1991) 12053.

[97] A.R. Beal, H.P. Hughes, W.Y. Liang, J. Phys. C-Solid State Phys., 8 (1975) 4236.

[98] M. Naito, S. Tanaka, J. Phys. Soc. Jpn., 51 (1982) 219.

[99] Y. Nishio, M. Shirai, N. Suzuki, K. Motizuki, J. Phys. Soc. Jpn., 63 (1994) 156.

[100] T. Straub, T. Finteis, R. Claessen, P. Steiner, S. Hufner, P. Blaha, C.S. Oglesby, E.

Bucher, Phys. Rev. Lett., 82 (1999) 4504.

[101] R.A. Bromley, Phys. Lett. A, A33 (1970) 242.

[102] R.A. Bromley, A.D. Yoffe, R.B. Murray, J. Phys. C Solid State Phys., 5 (1972) 759.

[103] T.K. Wieting, Schluter, M., in: E. Mooser (Ed.) Physics and Chemistry of Materials with Layered Structures, D.Reidel, Boston, 1979.

[104] T. Boker, R. Severin, A. Muller, C. Janowitz, R. Manzke, D. Voss, P. Kruger, A. Mazur, J. Pollmann, Phys. Rev. B, 64 (2001) 235305.

[105] T. Cheiwchanchamnangij, W.R.L. Lambrecht, Phys. Rev. B, 85 (2012) 205302.

[106] H. Jiang, J. Phys. Chem. C, 116 (2012) 7664.

[107] A. Enyashin, S. Gemming, G. Seifert, European Phys. J.- Special Topics, 149 (2007) 103. 
[108] S. Lebegue, O. Eriksson, Phys. Rev. B, 79 (2009) 115409.

[109] H. Terrones, F. Lopez-Urias, M. Terrones, Sci. Rep., 3 (2013) 1549.

[110] A. Kuc, N. Zibouche, T. Heine, Phys. Rev. B, 83 (2011) 245213.

[111] Y. Ding, Y. Wang, J. Ni, L. Shi, S. Shi, W. Tang, Physica B-Condensed Matter, 406 (2011) 2254.

[112] T.S. Li, G.L. Galli, J. Phys. Chem. C, 111 (2007) 16192.

[113] A. Ramasubramaniam, D. Naveh, E. Towe, Phys. Rev. B, 84 (2011) 205325.

[114] A. Ramasubramaniam, Phys. Rev. B, 86 (2012) 115409.

[115] A. Kumar, P.K. Ahluwalia, European Phys. J. B, 85 (2012) 186.

[116] H.L. Shi, H. Pan, Y.W. Zhang, B.I. Yakobson, Phys. Rev. B, 87 (2013) 155304.

[117] D. Xiao, G.-B. Liu, W. Feng, X. Xu, W. Yao, Phys. Rev. Lett., 108 (2012) 196802.

[118] Z.Y. Zhu, Y.C. Cheng, U. Schwingenschlogl, Phys. Rev. B, 84 (2011) 153402.

[119] A. Molina-Sanchez, D. Sangalli, K. Hummer, A. Marini, L. Wirtz, Phys. Rev. B, 88 (2013) 045412.

[120] T. Cao, G. Wang, W.P. Han, H.Q. Ye, C.R. Zhu, J.R. Shi, Q. Niu, P.H. Tan, E.

Wang, B.L. Liu, J. Feng, Nat. Commun., 3 (2012) 887.

[121] K.F. Mak, K.L. He, J. Shan, T.F. Heinz, Nat. Nanotechnol., 7 (2012) 494.

[122] S.F. Wu, J.S. Ross, G.B. Liu, G. Aivazian, A. Jones, Z.Y. Fei, W.G. Zhu, D. Xiao,

W. Yao, D. Cobden, X.D. Xu, Nat. Phys., 9 (2013) 149.

[123] H.L. Zeng, J.F. Dai, W. Yao, D. Xiao, X.D. Cui, Nat. Nanotechnol., 7 (2012) 490.

[124] K.S. Novoselov, D. Jiang, F. Schedin, T.J. Booth, V.V. Khotkevich, S.V. Morozov, A.K. Geim, Proc. Natl. Acad. Sci. U. S. A., 102 (2005) 10451.

[125] W.J. Yu, Z. Li, H.L. Zhou, Y. Chen, Y. Wang, Y. Huang, X.F. Duan, Nat. Mater., $12(2013) 246$.

[126] J.N. Coleman, M. Lotya, A. O'Neill, S.D. Bergin, P.J. King, U. Khan, K. Young, A.

Gaucher, S. De, R.J. Smith, I.V. Shvets, S.K. Arora, G. Stanton, H.Y. Kim, K. Lee, G.T. Kim, G.S. Duesberg, T. Hallam, J.J. Boland, J.J. Wang, J.F. Donegan, J.C. Grunlan, G. Moriarty, A. Shmeliov, R.J. Nicholls, J.M. Perkins, E.M. Grieveson, K. Theuwissen, D.W. McComb, P.D. Nellist, V. Nicolosi, Science, 331 (2011) 568.

[127] K. Lee, H.Y. Kim, M. Lotya, J.N. Coleman, G.T. Kim, G.S. Duesberg, Adv. Mater., 23 (2011) 4178.

[128] K.G. Zhou, N.N. Mao, H.X. Wang, Y. Peng, H.L. Zhang, Angew. Chem. Int. Ed., 50 (2011) 10839.

[129] D. Voiry, H. Yamaguchi, J. Li, R. Silva, D.C.B. Alves, T. Fujita, M. Chen, T.

Asefa, V.B. Shenoy, G. Eda, M. Chhowalla, Nat Mater, (2013) DOI: 10.1038/NMAT3700.

[130] M.B. Dines, Mater. Res. Bull., 10 (1975) 287.

[131] Z.Y. Zeng, Z.Y. Yin, X. Huang, H. Li, Q.Y. He, G. Lu, F. Boey, H. Zhang, Angew. Chem. Int. Ed., 50 (2011) 11093.

[132] L.A. King, W. Zhao, M. Chhowalla, D.J. Riley, G. Eda, J. Mater. Chem. A, 1 (2013) 8935.

[133] A. Castellanos-Gomez, M. Barkelid, A.M. Goossens, V.E. Calado, H.S.J. van der Zant, G.A. Steele, Nano Lett., 12 (2012) 3187.

[134] H.-P. Komsa, J. Kotakoski, S. Kurasch, O. Lehtinen, U. Kaiser, A.V. Krasheninnikov, Phys. Rev. Lett., 109 (2012) 035503.

[135] K.S. Novoselov, A.K. Geim, S.V. Morozov, D. Jiang, Y. Zhang, S.V. Dubonos, I.V. 
Grigorieva, A.A. Firsov, Science, 306 (2004) 666.

[136] H. Li, G. Lu, Z.Y. Yin, Q.Y. He, Q. Zhang, H. Zhang, Small, 8 (2012) 682.

[137] Y.F. Chen, J.Y. Xi, D.O. Dumcenco, Z. Liu, K. Suenaga, D. Wang, Z.G. Shuai,

Y.S. Huang, L.M. Xie, ACS Nano, 7 (2013) 4610.

[138] B. Radisavljevic, A. Kis, Nat Mater, 12 (2013) 815.

[139] O. Lopez-Sanchez, D. Lembke, M. Kayci, A. Radenovic, A. Kis, Nat. Nanotechnol., 8 (2013) 497.

[140] M. Fontana, T. Deppe, A.K. Boyd, M. Rinzan, A.Y. Liu, M. Paranjape, P. Barbara,

Sci. Rep., 3 (2013) 1634.

[141] F.K. Perkins, A.L. Friedman, E. Cobas, P.M. Campbell, G.G. Jernigan, B.T. Jonker, Nano Lett., 13 (2013) 668.

[142] J.N. Coleman, Acc. Chem. Res., 46 (2013) 14.

[143] R.A. Gordon, D. Yang, E.D. Crozier, D.T. Jiang, R.F. Frindt, Phys. Rev. B, 65 (2002) 125407.

[144] N.I. Kovtyukhova, Y.X. Wang, A. Berkdemir, R. Cruz-Silva, M. Terrones, V.H. Crespi, T.E. Mallouk, Nat. Chem., 6 (2014) 957.

[145] X. Huang, Z. Zeng, S. Bao, M. Wang, X. Qi, Z. Fan, H. Zhang, Nat Commun, 4 (2013) 1444.

[146] Z. Zeng, C. Tan, X. Huang, S. Bao, H. Zhang, Energy \& Environ. Sci., 7 (2014) 797.

[147] C.-B. Ma, X. Qi, B. Chen, S. Bao, Z. Yin, X.-J. Wu, Z. Luo, J. Wei, H.-L. Zhang, H. Zhang, Nanoscale, 6 (2014) 5624.

[148] C. Xu, S. Peng, C. Tan, H. Ang, H. Tan, H. Zhang, Q. Yan, J. Mater. Chem. A, 2 (2014) 5597.

[149] S.X. Wu, Z.Y. Zeng, Q.Y. He, Z.J. Wang, S.J. Wang, Y.P. Du, Z.Y. Yin, X.P. Sun,

W. Chen, H. Zhang, Small, 8 (2012) 2264.

[150] C.F. Zhu, Z.Y. Zeng, H. Li, F. Li, C.H. Fan, H. Zhang, J. Am. Chem. Soc., 135 (2013) 5998.

[151] Y. Zhang, B. Zheng, C. Zhu, X. Zhang, C. Tan, H. Li, B. Chen, J. Yang, J. Chen, Y. Huang, L. Wang, H. Zhang, Adv. Mater., 27 (2015) 935.

[152] Z. Yin, B. Chen, M. Bosman, X. Cao, J. Chen, B. Zheng, H. Zhang, Small, 10 (2014) 3537.

[153] Y.J. Zhan, Z. Liu, S. Najmaei, P.M. Ajayan, J. Lou, Small, 8 (2012) 966.

[154] K.K. Liu, W.J. Zhang, Y.H. Lee, Y.C. Lin, M.T. Chang, C. Su, C.S. Chang, H. Li,

Y.M. Shi, H. Zhang, C.S. Lai, L.J. Li, Nano Lett., 12 (2012) 1538.

[155] Y.C. Lin, W.J. Zhang, J.K. Huang, K.K. Liu, Y.H. Lee, C.T. Liang, C.W. Chu, L.J.

Li, Nanoscale, 4 (2012) 6637.

[156] N. Perea-López, A.L. Elías, A. Berkdemir, A. Castro-Beltran, H.R. Gutiérrez, S.

Feng, R. Lv, T. Hayashi, F. López-Urías, S. Ghosh, B. Muchharla, S. Talapatra, H.

Terrones, M. Terrones, Adv. Funct. Mater., 23 (2013) 5511.

[157] Y.F. Yu, C. Li, Y. Liu, L.Q. Su, Y. Zhang, L.Y. Cao, Sci. Rep., 3 (2013) 1866.

[158] X.S. Wang, H.B. Feng, Y.M. Wu, L.Y. Jiao, J. Am. Chem. Soc., 135 (2013) 5304.

[159] Q. Ji, Y. Zhang, T. Gao, Y. Zhang, D. Ma, M. Liu, Y. Chen, X. Qiao, P.-H. Tan, M.

Kan, J. Feng, Q. Sun, Z. Liu, Nano Lett., 13 (2013) 3870.

[160] J.V. Lauritsen, J. Kibsgaard, S. Helveg, H. Topsoe, B.S. Clausen, E. Laegsgaard, F.

Besenbacher, Nat. Nanotechnol., 2 (2007) 53. 
[161] Y.H. Lee, X.Q. Zhang, W.J. Zhang, M.T. Chang, C.T. Lin, K.D. Chang, Y.C. Yu, J.T.W. Wang, C.S. Chang, L.J. Li, T.W. Lin, Adv. Mater., 24 (2012) 2320.

[162] A.M. van der Zande, P.Y. Huang, D.A. Chenet, T.C. Berkelbach, Y.M. You, G.H. Lee, T.F. Heinz, D.R. Reichman, D.A. Muller, J.C. Hone, Nat. Mater., 12 (2013) 554.

[163] S. Najmaei, Z. Liu, W. Zhou, X.L. Zou, G. Shi, S.D. Lei, B.I. Yakobson, J.C. Idrobo, P.M. Ajayan, J. Lou, Nat. Mater., 12 (2013) 754.

[164] Y.H. Lee, L.L. Yu, H. Wang, W.J. Fang, X. Ling, Y.M. Shi, C.T. Lin, J.K. Huang, M.T. Chang, C.S. Chang, M. Dresselhaus, T. Palacios, L.J. Li, J. Kong, Nano Lett., 13 (2013) 1852.

[165] Z.Y. Wang, H. Li, Z. Liu, Z.J. Shi, J. Lu, K. Suenaga, S.K. Joung, T. Okazaki, Z.N. Gu, J. Zhou, Z.X. Gao, G.P. Li, S. Sanvito, E.G. Wang, S. Iijima, J. Am. Chem. Soc., 132 (2010) 13840.

[166] Z.Y. Wang, K.K. Zhao, H. Li, Z. Liu, Z.J. Shi, J. Lu, K. Suenaga, S.K. Joung, T. Okazaki, Z.X. Jin, Z.N. Gu, Z.X. Gao, S. Iijima, J. Mater. Chem., 21 (2011) 171.

[167] S.F. Wu, C.M. Huang, G. Aivazian, J.S. Ross, D.H. Cobden, X.D. Xu, ACS Nano, 7 (2013) 2768.

[168] H. Matte, A. Gomathi, A.K. Manna, D.J. Late, R. Datta, S.K. Pati, C.N.R. Rao, Angew. Chem. Int. Ed., 49 (2010) 4059.

[169] H.W. Zhu, C.L. Xu, D.H. Wu, B.Q. Wei, R. Vajtai, P.M. Ajayan, Science, 296 (2002) 884.

[170] R.T. Lv, T.X. Cui, M.S. Jun, Q. Zhang, A.Y. Cao, D.S. Su, Z.J. Zhang, S.H. Yoon, J. Miyawaki, I. Mochida, F.Y. Kang, Adv. Funct. Mater., 21 (2011) 999.

[171] X. Li, W. Cai, J. An, S. Kim, J. Nah, D. Yang, R. Piner, A. Velamakanni, I. Jung, E.

Tutuc, S.K. Banerjee, L. Colombo, R.S. Ruoff, Science, 324 (2009) 1312.

[172] R. Lv, Q. Li, A.R. Botello-Mendez, T. Hayashi, B. Wang, A. Berkdemir, Q.Z. Hao,

A.L. Elias, R. Cruz-Silva, H.R. Gutierrez, Y.A. Kim, H. Muramatsu, J. Zhu, M. Endo,

H. Terrones, J.C. Charlier, M.H. Pan, M. Terrones, Sci. Rep., 2 (2012) 586.

[173] D. Golberg, Y. Bando, C.C. Tang, C.Y. Zhi, Adv. Mater., 19 (2007) 2413.

[174] L. Song, L.J. Ci, H. Lu, P.B. Sorokin, C.H. Jin, J. Ni, A.G. Kvashnin, D.G.

Kvashnin, J. Lou, B.I. Yakobson, P.M. Ajayan, Nano Lett., 10 (2010) 3209.

[175] X.-Q. Zhang, C.-H. Lin, Y.-W. Tseng, K.-H. Huang, Y.-H. Lee, Nano Lett., 15 (2015) 410.

[176] Y. Gong, J. Lin, X. Wang, G. Shi, S. Lei, Z. Lin, X. Zou, G. Ye, R. Vajtai, B.I. Yakobson, H. Terrones, M. Terrones, B.K. Tay, J. Lou, S.T. Pantelides, Z. Liu, W.

Zhou, P.M. Ajayan, Nat. Mater., 13 (2014) 1135.

[177] M.-H. Chiu, M.-Y. Li, W. Zhang, W.-T. Hsu, W.-H. Chang, M. Terrones, H. Terrones, L.-J. Li, ACS Nano, 8 (2014) 9649.

[178] M. Remskar, A. Mrzel, Z. Skraba, A. Jesih, M. Ceh, J. Demsar, P. Stadelmann, F.

Levy, D. Mihailovic, Science, 292 (2001) 479.

[179] Y.Y. Peng, Z.Y. Meng, C. Zhong, J. Lu, W.C. Yu, Y.B. Jia, Y.T. Qian, Chem Lett, 8 (2001) 772.

[180] J.-t. Jang, S. Jeong, J.-w. Seo, M.-C. Kim, E. Sim, Y. Oh, S. Nam, B. Park, J.

Cheon, J. Am. Chem. Soc., 133 (2011) 7636.

[181] J.L. Verble, T.J. Wieting, Phys. Rev. Lett., 25 (1970) 362.

[182] J.L. Verble, T.J. Wietling, P.R. Reed, Solid State Commun., 11 (1972) 941.

[183] T.J. Wieting, A. Grisel, F. Levy, Physica B \& C, 99 (1980) 337. 
[184] C. Sourisseau, F. Cruege, M. Fouassier, M. Alba, Chem. Phys., 150 (1991) 281.

[185] A.M. Stacy, D.T. Hodul, J. Phys. Chem. Solids, 46 (1985) 405.

[186] M.S. Dresselhaus, G. Dresselhaus, R. Saito, A. Jorio, Phys. Rep.-Rev. Sec. Phys.

Lett., 409 (2005) 47.

[187] G.L. Frey, R. Tenne, M.J. Matthews, M.S. Dresselhaus, G. Dresselhaus, J. Mater.

Res., 13 (1998) 2412.

[188] C. Lee, H. Yan, L.E. Brus, T.F. Heinz, J. Hone, S. Ryu, ACS Nano, 4 (2010) 2695.

[189] A. Molina-Sanchez, L. Wirtz, Phys. Rev. B, 84 (2011) 155413.

[190] H. Sahin, S. Tongay, S. Horzum, W. Fan, J. Zhou, J. Li, J. Wu, F.M. Peeters, Phys.

Rev. B, 87 (2013) 165409.

[191] H.L. Zeng, G.B. Liu, J.F. Dai, Y.J. Yan, B.R. Zhu, R.C. He, L. Xie, S.J. Xu, X.H.

Chen, W. Yao, X.D. Cui, Sci. Rep., 3 (2013) 1608.

[192] Y.Y. Zhao, X. Luo, H. Li, J. Zhang, P.T. Araujo, C.K. Gan, J. Wu, H. Zhang, S.Y.

Quek, M.S. Dresselhaus, Q.H. Xiong, Nano Lett., 13 (2013) 1007.

[193] S. Najmaei, Z. Liu, P.M. Ajayan, J. Lou, Appl. Phys. Lett., 100 (2012) 013106.

[194] M.A. Pimenta, E. del Corro, B.R. Carvalho, C. Fantini, L.M. Malard, Acc. Chem. Res., 48 (2015) 41.

[195] B.R. Carvalho, L.M. Malard, J.M. Alves, C. Fantini, M.A. Pimenta, Phys. Rev. Lett., 114 (2015) 136403.

[196] E. del Corro, H. Terrones, A. Elias, C. Fantini, S. Feng, N. Minh An, T.E. Mallouk,

M. Terrones, M.A. Pimenta, ACS Nano, 8 (2014) 9629.

[197] G. Plechinger, S. Heydrich, J. Eroms, D. Weiss, C. Schuller, T. Korn, Appl. Phys. Lett., 101 (2012) 101906.

[198] G. Plechinger, S. Heydrich, M. Hirmer, F.X. Schrettenbrunner, D. Weiss, J. Eroms,

C. Schuller, T. Korn, Nanoengineering: Fabrication, Properties, Optics, and Devices

Ix. Proceedings of SPIE 8463 (2012).

[199] H. Terrones, E. Del Corro, S. Feng, J.M. Poumirol, D. Rhodes, D. Smirnov, N.R.

Pradhan, Z. Lin, M.A.T. Nguyen, A.L. Elias, T.E. Mallouk, L. Balicas, M.A. Pimenta,

M. Terrones, Sci. Rep., 4 (2014) 4215.

[200] A.C. Ferrari, J.C. Meyer, V. Scardaci, C. Casiraghi, M. Lazzeri, F. Mauri, S.

Piscanec, D. Jiang, K.S. Novoselov, S. Roth, A.K. Geim, Phys. Rev. Lett., 97 (2006) 187401.

[201] P. Venezuela, M. Lazzeri, F. Mauri, Phys. Rev. B, 84 (2011) 035433.

[202] C. Thomsen, S. Reich, Phys. Rev. Lett., 85 (2000) 5214.

[203] J. Maultzsch, S. Reich, C. Thomsen, Phys. Rev. B, 70 (2004) 155403.

[204] S. Reich, C. Thomsen, Phil. Trans. Royal Soc. A-Mathematical Phys. Eng. Sci., 362 (2004) 2271.

[205] Y.J. Zhang, J.T. Ye, Y. Matsuhashi, Y. Iwasa, Nano Lett., 12 (2012) 1136.

[206] S. Das, H.Y. Chen, A.V. Penumatcha, J. Appenzeller, Nano Lett., 13 (2013) 100.

[207] W.J. Zhang, C.P. Chuu, J.K. Huang, C.H. Chen, M.L. Tsai, Y.H. Chang, C.T.

Liang, Y.Z. Chen, Y.L. Chueh, J.H. He, M.Y. Chou, L.J. Li, Sci. Rep., 4 (2014) 3826.

[208] S.H. Lee, D. Lee, W.S. Hwang, E. Hwang, D. Jena, W.J. Yoo, Appl. Phys. Lett., 104 (2014) 193113.

[209] N. Perea-López, Z. Lin, N.R. Pradhan, A. Iñiguez-Rábago, A.L. Elías, A. McCreary, J. Lou, P.M. Ajayan, H. Terrones, L. Balicas, M. Terrones, 2D Mater., 1 (2014) 011004. 
[210] T. Korn, S. Heydrich, M. Hirmer, J. Schmutzler, C. Schuller, Appl. Phys. Lett., 99 (2011) 102109.

[211] G. Plechinger, F.X. Schrettenbrunner, J. Eroms, D. Weiss, C. Schuller, T. Korn, Phys. Status Solidi-R, 6 (2012) 126.

[212] Y.J. Gong, Z. Liu, A.R. Lupini, G. Shi, J.H. Lin, S. Najmaei, Z. Lin, A.L. Elias, A. Berkdemir, G. You, H. Terrones, M. Terrones, R. Vajtai, S.T. Pantelides, S.J. Pennycook, J. Lou, W. Zhou, P.M. Ajayan, Nano Lett., 14 (2014) 442.

[213] Z. Lin, M. Thee, A.L. Elias, S. Feng, C. Zhou, K. Fujisawa, N. Perea-Lopez, H. Terrones, M. Terrones, APL Mater., 2 (2014) 092514.

[214] R. Wang, B.A. Ruzicka, N. Kumar, M.Z. Bellus, H.Y. Chiu, H. Zhao, Phys. Rev. B, 86 (2012) 045406.

[215] J.-K. Huang, J. Pu, C.-P. Chuu, C.-L. Hsu, M.-H. Chiu, Z.-Y. Juang, Y.-H. Chang, W.-H. Chang, Y. Iwasa, M.-Y. Chou, T. Takenobu, L.-J. Li, arXiv, (2013) $1304.7365 \mathrm{v} 1$.

[216] D. Xiao, W. Yao, Q. Niu, Phys. Rev. Lett., 99 (2007) 236809.

[217] W. Yao, D. Xiao, Q. Niu, Phys. Rev. B, 77 (2008) 235406.

[218] A. Rycerz, J. Tworzydlo, C.W.J. Beenakker, Nat. Phys., 3 (2007) 172.

[219] A. Rycerz, Phys. Status Solidi A, 205 (2008) 1281.

[220] Z.Y. Zeng, T. Sun, J.X. Zhu, X. Huang, Z.Y. Yin, G. Lu, Z.X. Fan, Q.Y. Yan, H.H. Hng, H. Zhang, Angew. Chem. Int. Ed., 51 (2012) 9052.

[221] G. Sallen, L. Bouet, X. Marie, G. Wang, C.R. Zhu, W.P. Han, Y. Lu, P.H. Tan, T. Amand, B.L. Liu, B. Urbaszek, Phys. Rev. B, 86 (2012) 081301.

[222] A.M. Jones, H. Yu, N.J. Ghimire, S. Wu, G. Aivazian, J.S. Ross, B. Zhao, J. Yan, D.G. Mandrus, D. Xiao, W. Yao, X. Xu, Nat. Nanotechnol., 8 (2013) 634.

[223] Y.X. Lin, X. Ling, L.L. Yu, S.X. Huang, A.L. Hsu, Y.H. Lee, J. Kong, M.S.

Dressehaus, T. Palacios, Nano Lett., 14 (2014) 5569.

[224] Z.R. Gong, G.B. Liu, H.Y. Yu, D. Xiao, X.D. Cui, X.D. Xu, W. Yao, Nat. Commun., 4 (2013) 2053.

[225] G.A. Wagoner, P.D. Persans, E.A. Van Wagenen, G.M. Korenowski, J. Optical Soc. Am. B-Optical Phys., 15 (1998) 1017.

[226] G. Cetnarowski, G.W. Leach, Langmuir, 22 (2006) 8995.

[227] L.M. Malard, T.V. Alencar, A.P.M. Barboza, K.F. Mak, A.M. de Paula, Phys. Rev.

B, 87 (2013) 201401.

[228] N. Kumar, S. Najmaei, Q.N. Cui, F. Ceballos, P.M. Ajayan, J. Lou, H. Zhao, Phys.

Rev. B, 87 (2013) 161403(R).

[229] K. He, C. Poole, K.F. Mak, J. Shan, Nano Lett., 13 (2013) 2931.

[230] Y.L. Li, Y. Rao, K.F. Mak, Y.M. You, S.Y. Wang, C.R. Dean, T.F. Heinz, Nano Lett., 13 (2013) 3329.

[231] G. Eda, S.A. Maier, ACS Nano, 7 (2013) 5660.

[232] C. Janisch, Y. Wang, D. Ma, N. Mehta, A.L. Elias, N. Perea-Lopez, M. Terrones, V. Crespi, Z. Liu, Sci. Rep., 4 (2014) 5530.

[233] S. Wang, H. Yu, H. Zhang, A. Wang, M. Zhao, Y. Chen, L. Mei, J. Wang, Adv. Mater., 26 (2014) 3538.

[234] K. Wang, J. Wang, J. Fan, M. Lotya, A. O’Neill, D. Fox, Y. Feng, X. Zhang, B. Jiang, Q. Zhao, H. Zhang, J.N. Coleman, L. Zhang, W.J. Blau, ACS Nano, 7 (2013) 9260. 
[235] K.-G. Zhou, M. Zhao, M.-J. Chang, Q. Wang, X.-Z. Wu, Y. Song, H.-L. Zhang, Small, 11 (2015) 694.

[236] K.K. Fung, J.W. Steeds, J.A. Eades, Physica B \& C, 99 (1980) 47.

[237] J.A. Wilson, F.J. Di Salvo, S. Mahajan, Adv. Phys., 50 (2001) 1171.

[238] L. Ci, L. Song, C.H. Jin, D. Jariwala, D.X. Wu, Y.J. Li, A. Srivastava, Z.F. Wang,

K. Storr, L. Balicas, F. Liu, P.M. Ajayan, Nat. Mater., 9 (2010) 430.

[239] C. Liu, O. Singh, P. Joensen, A.E. Curzon, R.F. Frindt, Thin Solid Films, 113 (1984) 165.

[240] H.P. Komsa, S. Kurasch, O. Lehtinen, U. Kaiser, A.V. Krasheninnikov, Phys. Rev.

B, 88 (2013) 035301.

[241] S.J. Haigh, A. Gholinia, R. Jalil, S. Romani, L. Britnell, D.C. Elias, K.S. Novoselov,

L.A. Ponomarenko, A.K. Geim, R. Gorbachev, Nat. Mater., 11 (2012) 764.

[242] G. Binning, H. Rohrer, C. Gerber, E. Weibel, Phys. Rev. Lett., 49 (1982) 57.

[243] M. Weimer, J. Kramar, C. Bai, J.D. Baldeschwieler, W.J. Kaiser, J. Vac. Sci.

Technol. A-Vac. Surf. Films, 6 (1988) 336.

[244] S. Helveg, J.V. Lauritsen, E. Laegsgaard, I. Stensgaard, J.K. Norskov, B.S. Clausen,

H. Topsoe, F. Besenbacher, Phys. Rev. Lett., 84 (2000) 951.

[245] E.Y. Polyakova, K.T. Rim, D. Eom, K. Douglass, R.L. Opila, T.F. Heinz, A.V.

Teplyakov, G.W. Flynn, ACS Nano, 5 (2011) 6102.

[246] J.B. Park, B. Jaeckel, B.A. Parkinson, Langmuir, 22 (2006) 5334.

[247] M.M. Benameur, B. Radisavljevic, J.S. Heron, S. Sahoo, H. Berger, A. Kis,

Nanotechnology, 22 (2011) 125706.

[248] P. Nemes-Incze, Z. Osvath, K. Kamaras, L.P. Biro, Carbon, 46 (2008) 1435.

[249] Y. Kim, J.L. Huang, C.M. Lieber, Appl. Phys. Lett., 59 (1991) 3404.

[250] J.C. Bernede, Appl. Surf. Sci., 171 (2001) 15.

[251] M.A. Baker, R. Gilmore, C. Lenardi, W. Gissler, Appl. Surf. Sci., 150 (1999) 255.

[252] M. Bougouma, A. Batan, B. Guel, T. Segato, J.B. Legma, F. Reniers, M.P.

Delplancke-Ogletree, C. Buess-Herman, T. Doneux, J. Cryst. Growth, 363 (2013) 122.

[253] J. Pouzet, J.C. Bernede, A. Khellil, H. Essaidi, S. Benhida, Thin Solid Films, 208 (1992) 252.

[254] P.P. Hankare, A.H. Manikshete, D.J. Sathe, P.A. Chate, A.A. Patil, K.M. Garadkar,

J. Alloys Compd., 479 (2009) 657.

[255] J. Tauc, Grigorov.R, A. Vancu, Phys. Status Solidi, 15 (1966) 627.

[256] H. Li, J. Wu, X. Huang, G. Lu, J. Yang, X. Lu, Q. Xiong, H. Zhang, ACS Nano, 7 (2013) 10344.

[257] Y.J. Zhang, J.T. Ye, Y. Yornogida, T. Takenobu, Y. Iwasa, Nano Lett., 13 (2013) 3023.

[258] Z.Y. Yin, H. Li, H. Li, L. Jiang, Y.M. Shi, Y.H. Sun, G. Lu, Q. Zhang, X.D. Chen, H. Zhang, ACS Nano, 6 (2012) 74.

[259] H.S. Lee, S.W. Min, Y.G. Chang, M.K. Park, T. Nam, H. Kim, J.H. Kim, S. Ryu, S. Im, Nano Lett., 12 (2012) 3695.

[260] W.S. Hwang, M. Remskar, R.S. Yan, V. Protasenko, K. Tahy, S.D. Chae, P. Zhao,

A. Konar, H.L. Xing, A. Seabaugh, D. Jena, Appl. Phys. Lett., 101 (2012) 013107.

[261] W. Choi, M.Y. Cho, A. Konar, J.H. Lee, G.B. Cha, S.C. Hong, S. Kim, J. Kim, D.

Jena, J. Joo, S. Kim, Adv. Mater., 24 (2012) 5832.

[262] L. Britnell, R.M. Ribeiro, A. Eckmann, R. Jalil, B.D. Belle, A. Mishchenko, Y.J. 
Kim, R.V. Gorbachev, T. Georgiou, S.V. Morozov, A.N. Grigorenko, A.K. Geim, C.

Casiraghi, A.H.C. Neto, K.S. Novoselov, Science, 340 (2013) 1311.

[263] B. Radisavljevic, M.B. Whitwick, A. Kis, Appl. Phys. Lett., 102 (2013) 043103.

[264] B. Radisavljevic, M.B. Whitwick, A. Kis, ACS Nano, 5 (2011) 9934.

[265] J. Pu, Y. Yomogida, K.K. Liu, L.J. Li, Y. Iwasa, T. Takenobu, Nano Lett., 12 (2012) 4013.

[266] L.Z. Kou, T. Frauenheim, C.F. Chen, J. Phys. Chem. Lett., 4 (2013) 1730.

[267] D.-S. Tsai, K.-K. Liu, D.-H. Lien, M.-L. Tsai, C.-F. Kang, C.-A. Lin, L.-J. Li, J.-H.

He, ACS Nano, 7 (2013) 3905.

[268] Q.Y. He, Z.Y. Zeng, Z.Y. Yin, H. Li, S.X. Wu, X. Huang, H. Zhang, Small, 8 (2012) 2994.

[269] H. Liu, M.W. Si, S. Najmaei, A.T. Neal, Y.C. Du, P.M. Ajayan, J. Lou, P.D.D. Ye,

Nano Lett., 13 (2013) 2640.

[270] M. Amani, M.L. Chin, A.G. Birdwell, T.P. O'Regan, S. Najmaei, Z. Liu, P.M.

Ajayan, J. Lou, M. Dubey, Appl. Phys. Lett., 102 (2013) 193107.

[271] N.R. Pradhan, D. Rhodes, S. Memaran, J.M. Poumirol, D. Smirnov, S. Talapatra, S.

Feng, N. Perea-Lopez, A.L. Elias, M. Terrones, P.M. Ajayan, L. Balicas, Sci. Rep., (2015) Accepted.

[272] N.R. Pradhan, D. Rhodes, Q. Zhang, S. Talapatra, M. Terrones, P.M. Ajayan, L.

Balicas, Appl. Phys. Lett., 102 (2013) 123105.

[273] D. Braga, I.G. Lezama, H. Berger, A.F. Morpurgo, Nano Lett., 12 (2012) 5218.

[274] N.R. Pradhan, D. Rhodes, S. Feng, Y. Xin, S. Memaran, B.-H. Moon, H. Terrones,

M. Terrones, L. Balicas, ACS Nano, 8 (2014) 5911.

[275] M. Bernardi, M. Palummo, J.C. Grossman, Nano Lett., 13 (2013) 3664.

[276] W.J. Yu, Y. Liu, H.L. Zhou, A.X. Yin, Z. Li, Y. Huang, X.F. Duan, Nat.

Nanotechnol., 8 (2013) 952.

[277] K. Jiao, C. Duan, X. Wu, J. Chen, Y. Wang, Y. Chen, Phys. Chem. Chem. Phys.,

17 (2015) 8182.

[278] Y. Tsuboi, F. Wang, D. Kozawa, K. Funahashi, S. Mouri, Y. Miyauchi, T.

Takenobu, K. Matsuda, (2015) arXiv:1503.05380.

[279] D.J. Late, Y.K. Huang, B. Liu, J. Acharya, S.N. Shirodkar, J.J. Luo, A.M. Yan, D.

Charles, U.V. Waghmare, V.P. Dravid, C.N.R. Rao, ACS Nano, 7 (2013) 4879.

[280] Q.F. Zhang, K. Yu, B. Zhao, Y. Wang, C.Q. Song, S.C. Li, H.H. Yin, Z.L. Zhang,

Z.Q. Zhu, RSC Adv, 3 (2013) 10994.

[281] J. Liu, Z. Zeng, X. Cao, G. Lu, L.-H. Wang, Q.-L. Fan, W. Huang, H. Zhang, Small, 8 (2012) 3517.

[282] Z. Yin, Z. Zeng, J. Liu, Q. He, P. Chen, H. Zhang, Small, 9 (2013) 727.

[283] R.S. Sundaram, M. Engel, A. Lombardo, R. Krupke, A.C. Ferrari, P. Avouris, M.

Steiner, Nano Lett., 13 (2013) 1416.

[284] K.A.N. Duerloo, M.T. Ong, E.J. Reed, J. Phys. Chem. Lett., 3 (2012) 2871.

[285] W.Z. Wu, L. Wang, Y.L. Li, F. Zhang, L. Lin, S.M. Niu, D. Chenet, X. Zhang, Y.F.

Hao, T.F. Heinz, J. Hone, Z.L. Wang, Nature, 514 (2014) 470.

[286] C. Janisch, N. Mehta, D. Ma, A.L. Elias, N. Perea-Lopez, M. Terrones, Z.W. Liu, Opt. Lett., 39 (2014) 383.

[287] A. Winchester, S. Ghosh, S. Feng, A.L. Elias, T. Mallouk, M. Terrones, S. Talapatra, ACS Appl. Mater. Interfaces, 6 (2014) 2125. 
[288] L. Margulis, G. Salitra, R. Tenne, M. Talianker, Nature, 365 (1993) 113.

[289] G. Seifert, H. Terrones, M. Terrones, G. Jungnickel, T. Frauenheim, Phys. Rev.

Lett., 85 (2000) 146.

[290] H.P. Komsa, J. Kotakoski, S. Kurasch, O. Lehtinen, U. Kaiser, A.V.

Krasheninnikov, Phys. Rev. Lett., 109 (2012) 035503.

[291] W. Zhou, X. Zou, S. Najmaei, Z. Liu, Y. Shi, J. Kong, J. Lou, P.M. Ajayan, B.I.

Yakobson, J.-C. Idrobo, Nano Lett., 13 (2013) 2615.

[292] Y. Zhang, Y. Zhang, Q. Ji, J. Ju, H. Yuan, J. Shi, T. Gao, D. Ma, M. Liu, Y. Chen,

X. Song, H.Y. Hwang, Y. Cui, Z. Liu, ACS Nano, 7 (2013) 8963.

[293] X.L. Zou, Y.Y. Liu, B.I. Yakobson, Nano Lett., 13 (2013) 253.

[294] A. Azizi, X.L. Zou, P. Ercius, Z.H. Zhang, A.L. Elias, N. Perea-Lopez, G. Stone, M.

Terrones, B.I. Yakobson, N. Alem, Nat. Commun., 5 (2014) 4867.

[295] D.O. Dumcenco, H. Kobayashi, Z. Liu, Y.S. Huang, K. Suenaga, Nat. Commun., 4 (2013).

[296] H. Wang, L. Yu, Y.-H. Lee, Y. Shi, A. Hsu, M.L. Chin, L.-J. Li, M. Dubey, J.

Kong, T. Palacios, Nano Lett., 12 (2012) 4674.

[297] H.P. Komsa, A.V. Krasheninnikov, Phys. Rev. B, 88 (2013) 085318.

[298] J. Kang, J. Li, S.-S. Li, J.-B. Xia, L.-W. Wang, Nano Lett., 13 (2013) 5485.

[299] M. Fujita, K. Wakabayashi, K. Nakada, K. Kusakabe, J. Phys. Soc. Jpn., 65 (1996) 1920.

[300] K. Nakada, M. Fujita, G. Dresselhaus, M.S. Dresselhaus, Phys. Rev. B, 54 (1996) 17954.

[301] M. Terrones, A.R. Botello-Mendez, J. Campos-Delgado, F. Lopez-Urias, Y.I. Vega-Cantu, F.J. Rodriguez-Macias, A.L. Elias, E. Munoz-Sandoval, A.G.

Cano-Marquez, J.C. Charlier, H. Terrones, Nano Today, 5 (2010) 351.

[302] M.V. Bollinger, J.V. Lauritsen, K.W. Jacobsen, J.K. Norskov, S. Helveg, F.

Besenbacher, Phys. Rev. Lett., 87 (2001) 196803.

[303] S.S. Chou, M. De, J. Kim, S. Byun, C. Dykstra, J. Yu, J.X. Huang, V.P. Dravid, J. Am. Chem. Soc., 135 (2013) 4584.

[304] C. Backes, N.C. Berner, X. Chen, P. Lafargue, P. LaPlace, M. Freeley, G.S. Duesberg, J.N. Coleman, A.R. McDonald, Angew. Chem. Int. Ed., 54 (2015) 2638.

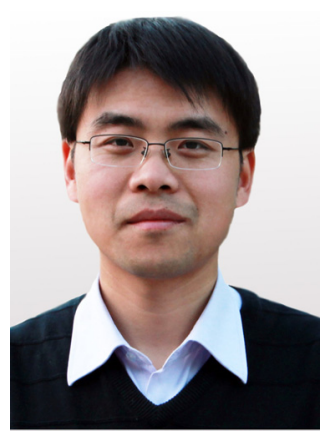

Ruitao Lv received his PhD in 2009 from Tsinghua University, China. $\mathrm{He}$ has been a visiting researcher in Kyushu University (Japan) and a postdoctoral researcher in Penn State University (USA). He joined Tsinghua University as an Assistant Professor since February of 2013. His research interests include the synthesis, characterization and applications of carbon nanomaterials (e.g. carbon nanotubes, graphene) and 2-D layered materials. 


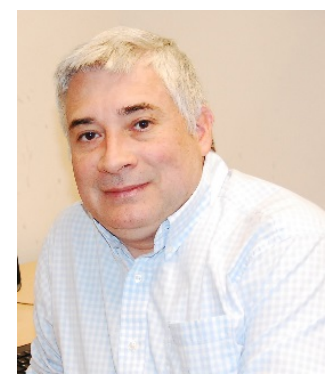

Humberto Terrones carried out his $\mathrm{PhD}$ at the University of London (Birkbeck College) under the supervision of Prof. Alan L. Mackay (FRS). In 1991 Humberto Terrones and Alan Mackay were the first in introducing the concept of curvature in graphite and graphene proposing graphitic structures with negative Gaussian curvature called Schwarzites. Later, these ideas were implemented to understand defects in 2-D graphitic systems and were extended to transition metal dichalcogenides (TMDs). He is interested in the electronic, optical, mechanical and chemical properties of few layered 2-D materials as well as using them as building blocks to form new 3-D nanostructures. He is a member of the World Academy of Sciences for the Advancement of Science in Developing Countries (TWAS) and the Mexican Academy of Sciences. He has been invited professor in Belgium (UCL), Brazil (UFC), Japan (Shinshu), ORNL (USA), Penn State University (USA) and Sussex University (UK). At present, he is Rayleigh Endowed Chair professor of physics at Rensselaer Polytechnic Institute (USA).

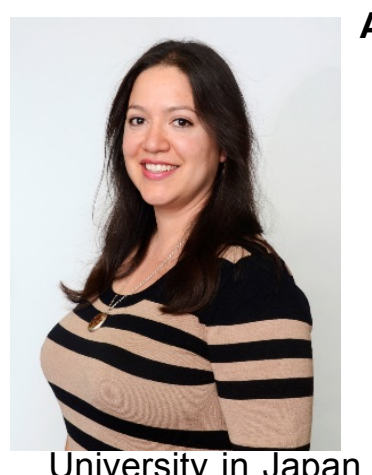

Ana Laura Elías received her Ph.D. degree in Nanoscience and Nanotechnology from IPICyT, in Mexico (2006). She has a Physics background, form her undergraduate degree (UASLP-Mexico, 2002). Ana Laura has visited several institutions for research stays, such as LANL (in New Mexico, USA), ASU (Tempe, AZ), MIT (Cambridge, MA), UNAM (Mexico City), Rice University (Houston, TX) and RPI (Troy, NY). She was a postdoctoral research fellow at Shinshu Ajayan). She has worked on the synthesis, characterization and applications of carbon nanomaterials. She is also involved in experimental research regarding other $2 \mathrm{D}$ and layer materials, such as semiconducting transition metal dichacogenides. Since 2010 , she is a Research Associate in the Department of the Pennsylvania State University (USA).

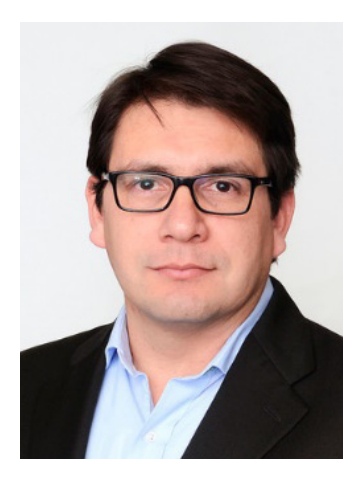

Néstor Perea-López received his PhD in Physics of Materials from CICESE (Mexico) in 2006. He was postdoctoral researcher in the University of California San Diego (USA) and IPICYT (Mexico). Since 2010 he works as research associate in Prof. Mauricio Terrones group at the physics department of the Pennsylvania State University (USA). Dr. Perea-Lopez's research interest is related to synthesis and 
characterization of carbon nanotubes, graphene and 2D semiconductors with special focus on electrical and optoelectronic applications.

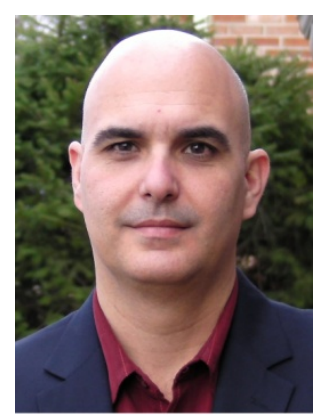

Humberto R. Gutiérrez received his PhD in Physics from The State University of Campinas (UNICAMP, SP Brazil) in 2001. He was a post-doctoral research associate at The Pennsylvania State University. In 2012 he joined The University of Louisville (Kentucky) as Assistant Professor. Dr. Gutierrez received the National Science Foundation CAREER award in 2015. His research interests are in nanoscale science, synthesis and characterization of nanostructures and 2-D materials for functional applications, and physical phenomena in low-dimensional systems.

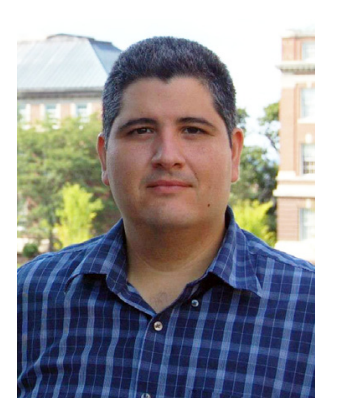

Eduardo Cruz Silva received his PhD in Applied Sciences in 2008 from IPICyT, in Mexico. He has been a postdoctoral researcher at Oak Ridge National Laboratory, University of Massachusetts Amherst, and Rensselaer Polytechnic Institute. He joined Penn State as a research associate in July 2013. His research Interests include the theory, modeling, and computer simulation of the electronic, structural, chemical, and quantum transport properties of low-dimensional materials.

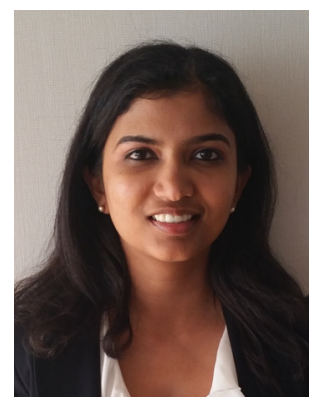

Lakshmy Pulickal Rajukumar received her B.Tech in Metallurgical and materials engineering from National Institute of Technology, Tiruchirappalli, India, in 2011. She is currently working with Prof.Mauricio Terrones at the Pennsylvania State University, USA towards her PhD degree in Materials science and engineering. Lakshmy's research focus includes synthesis and characterization of novel nanomaterials, particularly doped carbon nanotubes (CNTs), 3-D networks of CNTs, graphene-CNT hybrids as well as CVD synthesis of TMDs.

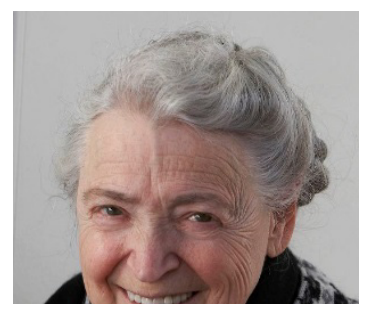

Mildred S. Dresselhaus joined the faculty at the Massachusetts 
Institute of Technology in 1967, and is an Institute Professor in the departments of physics and electrical engineering. Her research interests cover the area of materials physics generally, and layered material has been a main area of interest starting in 1960. Her interest in layered nanomaterials stated in 1973 with intercalation compounds. Her early work on layered transition metal dichalcogenides goes back to 1991. A leader in promoting opportunities for women in science and engineering, Dresselhaus received a Carnegie Foundation grant in 1973 to encourage women's study of traditionally male dominated fields, such as physics. In 1973, she was appointed to The Abby Rockefeller Mauze chair, an Institute-wide chair, endowed in support of the scholarship of women in science and engineering. A member of the National Academy of Sciences and Engineering in the US, Dresselhaus has received numerous awards and recognitions that include more 19 honorary degrees, the Kavli Prize in Nanoscience, The Presidential Medal of Freedom, The Enrico Fermi Award and many others.

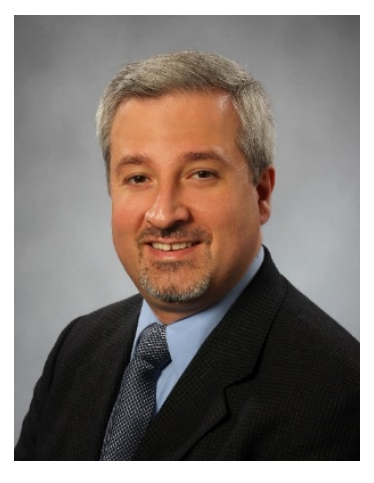

Mauricio Terrones Professor of Physics, Chemistry, and Materials Science \& Engineering at Penn State. He is currently the founding Director of the Center for 2-Dimensional and Layered Materials at Penn State since March 2013. He is a world leader in nanoscale layered materials including graphene, nitrides, and chalcogenides. $\mathrm{He}$ has published more than 380 publications in leading journals including Nature, Science, Nature Materials, Nature Nanotechnology, Nature Chemistry, Nature Communications, Physical Review Letters, ACS Nano, Nano Letters, Advanced Materials, etc. His work has been cited more than 20,000 times and his $\mathrm{H}$ index is 76 (Google Scholar 82). Mauricio obtained his doctorate degree in 1998 with Sir Prof. Harold W. Kroto (Nobel Laureate, FRS) from The University of Sussex. $\mathrm{He}$ has been recipient of the Mexican National Prize for Chemistry, the Javed Husain Prize, Albert Einstein medal from UNESCO, the TWAS Prize in Engineering Physics, and others. $\mathrm{He}$ is member of the Mexican Academy of Sciences, TWAS fellow and fellow of the American Association for the Advancement of Science (AAAS). Terrones is the principal editor of Carbon, Journal of Materials Research, 2D Materials and Materials Express. 


\section{GRAPHICAL ABSTRACT}

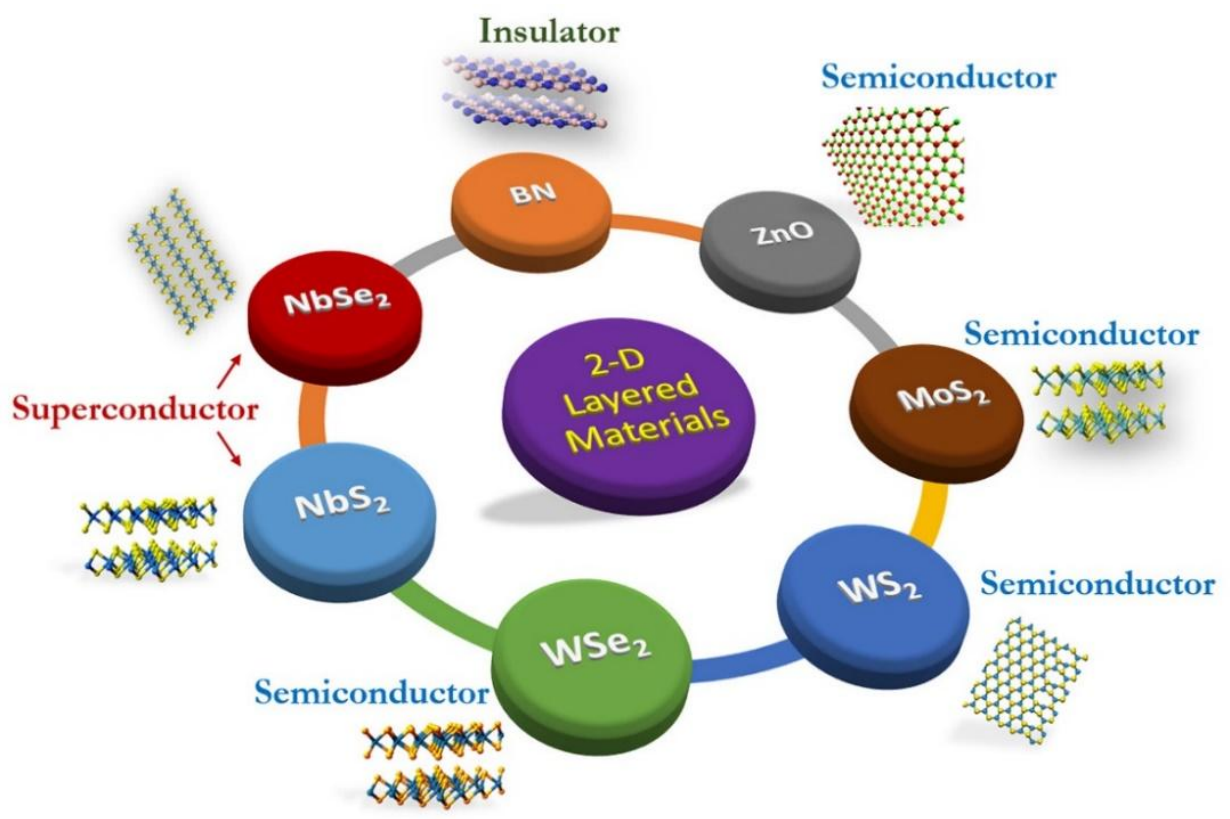

